GOVERNANCE AND THE EFFICIENCY

OF ECONOMIC SYSTEMS

GESY

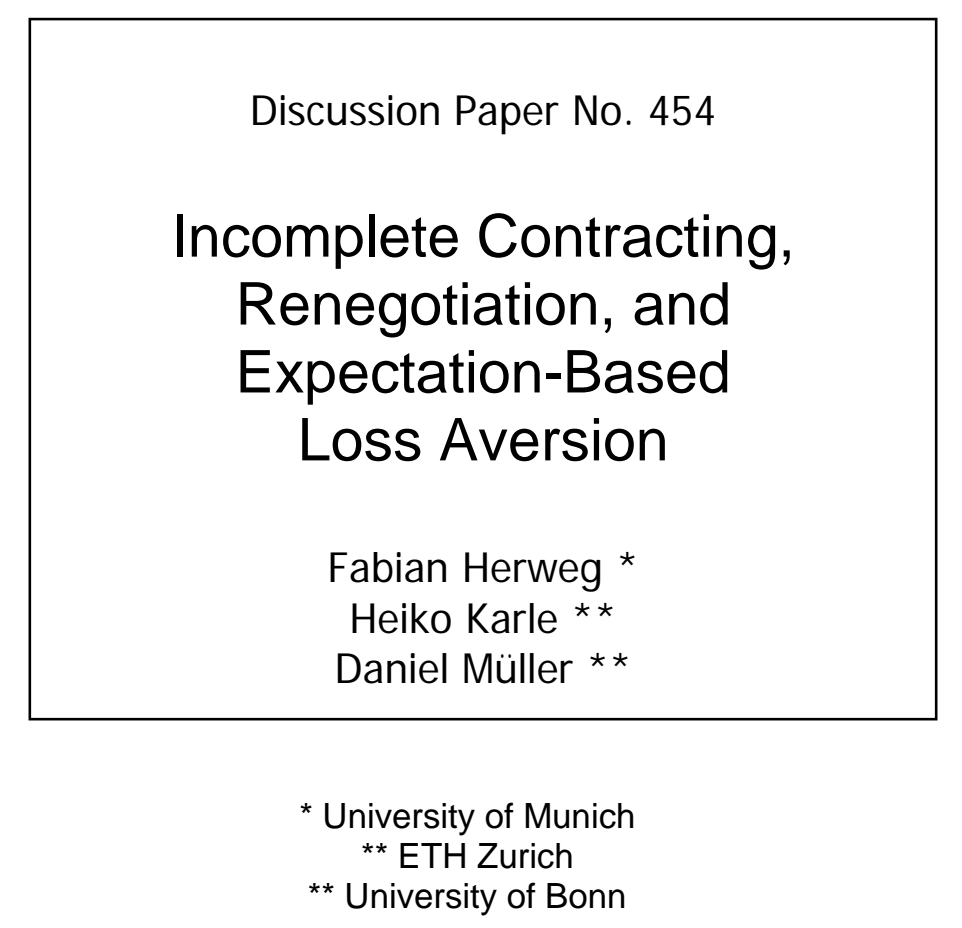

February 2014

Financial support from the Deutsche Forschungsgemeinschaft through SFB/TR 15 is gratefully acknowledged. 


\title{
Incomplete Contracting, Renegotiation, and Expectation-Based Loss Aversion*
}

\author{
FABIAN HERWEG HeIKO KARLE $^{\ddagger}$ AND DANIEL MÜLLER ${ }^{\S}$
}

February 13, 2014

\begin{abstract}
We consider a simple trading relationship between an expectation-based loss-averse buyer and profit-maximizing sellers. When writing a long-term contract the parties have to rely on renegotiations in order to ensure materially efficient trade ex post. The type of the concluded long-term contract affects the buyer's expectations regarding the outcome of renegotiation. If the buyer expects renegotiation always to take place, the parties are always able to implement the materially efficient good ex post. It can be optimal for the buyer, however, to expect that renegotiation does not take place. In this case, a good of too high quality or too low quality is traded ex post. Based on the buyer's expectation management, our theory provides a rationale for "employment contracts" in the absence of non-contractible investments. Moreover, in an extension with non-contractible investments, we show that loss aversion can reduce the hold-up problem.
\end{abstract}

JEL classification: C78; D03; D86

Keywords: Behavioral Contract Theory; Expectation-Based Loss Aversion; Incomplete Contracts; Renegotiation

\section{INTRODUCTION}

Despite the complexity of the respective trading environment, contracts observed in practice are often relatively simple. A possible reason for this could be indescribable contingencies,

${ }^{*}$ We thank Takeshi Murooka, Antonio Rosato, Klaus M. Schmidt, and Patrick Schmitz for helpful comments and suggestions. Furthermore, we have benefited from comments made by conference participants at the SFB Meeting at Bonn and from seminar audiences at LMU Munich, University of Zurich, University of Cologne, and Aarhus University. Fabian Herweg gratefully acknowledges financial support from the Deutsche Forschungsgemeinschaft through SFB/TR-15. Heiko Karle gratefully acknowledge financial support from the National Bank of Belgium (Research Grant, "The Impact of Consumer Loss Aversion on the Price Elasticity of Demand") and the ARC Grant "Market Evolution, Competition and Policy: Theory and Evidence". All errors are of course our own.

${ }^{\dagger}$ University of Munich, CESifo, and CEPR, Department of Economics, Ludwigstr. 28, D-80539 Munich, Germany, E-mail address: fabian.herweg@econ.lmu.de

${ }^{\ddagger}$ ETH Zurich, Center for Law and Economics, Haldeneggsteig 4, 8092 Zurich, Switzerland, E-mail address: hkarle@ethz.ch, Tel: +41-44-6326029.

$\S$ University of Bonn, Department of Economics, Adenauerallee 24-42, D-53113 Bonn, Germany, E-mail address: daniel.mueller@uni-bonn.de, Tel: +49-228-739212, Fax: +49-228-739212. 
which prevent the contracting parties from writing a fully state-dependent long-term contract. Instead, parties write a simple state-independent "incomplete" contract—e.g., a sales contract specifying a particular good to be delivered in the future by a seller to a buyer at a prespecified price. With the state of the world being relevant for the buyer's benefit from and the seller's cost for provision of a certain service, the parties then have to rely on renegotiations in order to implement the efficient service ex post.

The standard approach of the incomplete contracting literature, which assumes that the parties engage in efficient bargaining ex post à la Coase (1960) and therefore focuses on the ex ante inefficiencies caused by contractual incompleteness, recently has been challenged by behavioral approaches — most notably by Hart and Moore (2008). ${ }^{1}$ Hart and Moore were the first to point out that the initial contract can shape a reference point for the parties which affects the ex post outcome. We built on this main idea by positing that the buyer has reference-dependent preferences and that his reference point is affected by the concluded long-term contract. In contrast to Hart and Moore, our analysis has a strong focus on the outcome of renegotiations, which ever since have played a crucial role in the standard theory on incomplete contracts. In particular, we are interested in how the expectations regarding whether renegotiations will take place as well as regarding their outcome affects the likelihood of renegotiations to take place and their efficiency. We posit that the buyer is expectation-based loss averse according to Kőszegi and Rabin (2006). The buyer's reference point at the renegotiation stage is fully determined by his rational expectations formed ex ante when writing the long-term contract. Importantly, the long-term contract does not directly shape but indirectly influences the buyer's reference point by narrowing down his expectations regarding the outcome of renegotiations. If renegotiations take place, then this is perfectly anticipated by the parties. While this is a strong assumption, we believe that in situations where renegotiations are typically necessary in order to implement the efficient service it is reasonable to assume that the parties anticipate renegotiations to some degree and that this in turn is incorporated in their reference points. ${ }^{2}$

We consider a simple trading relationship where at some point in the future a buyer requires a service, which can be delivered by one of two sellers. The buyer can either sign a long-term contract today without knowing the ex post efficient service or engage in spot contracting in the future after the state of nature has been materialized. Ex ante there is competition between the sellers for the buyer. Ex post, when the long-term contract is renegotiated or spot contracting takes place the two parties face bilateral monopoly-i.e., within the time horizon a fundamental transformation in the sense of Williamson (1985) takes place. ${ }^{3}$ The novelty of our paper is

\footnotetext{
${ }^{1}$ For a synthesis of the classic literature on incomplete contracts see Bolton and Dewatripont (2005).

${ }^{2}$ Casual evidence that the expectations regarding whether renegotiations will take place affect the parties willingness indeed to renegotiate the contract ex post is that in some industries renegotiations are common while in others they are not. For instance, analyzing concession contracts in Latin American and Caribbean Countries, Guasch (2004) finds that roughly $75 \%$ of the water and sanitation concession contracts were renegotiated while less than $10 \%$ of the concession contracts in the electricity sector were renegotiated.

${ }^{3} \mathrm{~A}$ potential story is the following told by Hart and Moore (2008). The buyer organizes a wedding and the seller operates a catering service. Half a year before the wedding takes place there are many caterers, while a week before the wedding it is hard to find a new caterer. Even if the buyer does not sign a long-term contract ex ante,
} 
to assume that the buyer is expectation-based loss averse à la Köszegi and Rabin (2006). The buyer forms ex ante rational expectations about trade ex post, which shape a reference point for him separately in the "value" and the "price" dimension. In other words, the buyer feels a loss ex post if the price he has to pay exceeds his reference price or if the value he obtains from the delivered service is below his reference value. The buyer can be thought of as a layperson who engages in contracting and renegotiating only infrequently and suffers from loss aversion. The sellers, on the other hand, are assumed to be profit-maximizing professional traders who are used to trade and negotiate and do not suffer from loss aversion. ${ }^{4}$

Regarding the form that long-term contracts may take, we follow the traditional approach of Simon (1951) and compare a sales contract to an employment contract. A sales contract specifies a particular service to be delivered at a fixed price. Under an employment contract the price is also specified but one party is designated to freely choose the service within some specified limits. According to Simon, the advantage of an employment contact is rooted in its flexibility, whereas its disadvantage is that the party who is allowed to choose may exploit her/his trading partner. Usually it is argued, however, that the argument put forth by Simon is incomplete because it ignores the possibility of renegotiations. If Coasian bargaining is feasible ex post, there is no difference between these two types of long-term contracts. ${ }^{5}$ We show that in our setup these two types of contracts do not lead to the same expected gains from trade and thus indeed differ. The reason is that two kinds of inefficiencies may arise ex post. First, with the buyer being loss averse, efficient renegotiations do not always take place. Second, even if the efficient service is traded ex post, the losses incurred by the buyer depend on the concluded long-term contract-i.e, the necessary adjustment in prices during renegotiations can differ.

We establish that, irrespective of the type of the concluded long-term contract, if the buyer expects efficient renegotiations and this is correctly anticipated by the seller, then it is indeed optimal for the buyer to accept materially efficient renegotiations ex post-i.e., this constitutes a consistent plan. In other words, there always exists - as we will call it, borrowing the language of Kőszegi and Rabin (2006)—a subgame-perfect personal equilibrium (SP-PE) in which efficient renegotiations take place. Thus, in contrast to the conventional belief that loss aversion causes contractual stickiness, we show that this is not necessary the case if the reference point is determined by rational expectations.

Due to the self-fulfilling prophecy nature of the personal equilibrium (PE) concept, the equilibrium often is not unique. If this is the case, adopting the notion of preferred personal equilibrium (Kőszegi and Rabin, 2006), we presume that the buyer selects the plan among all credible plans that maximizes his expected utility. We call the equilibrium in which sellers correctly anticipate this expectation formation of the buyer subgame-perfect preferred personal equilibrium

\footnotetext{
he may reach an informal agreement with one seller so that there is a bilateral monopoly ex post.

${ }^{4}$ List (2011) points out that laypersons are affected more intensively by loss aversion than professional traders who are more used to trade and renegotiate.

${ }^{5}$ If the parties can make relationship-specific investments, the performance of sales contracts and employment contracts is different even when Coasian bargaining takes place-see, e.g., Bolton and Dewatripont (2005).
} 
(SP-PPE). In the SP-PPE efficient renegotiation does not always take place-in particular when the buyer is highly loss averse. The intuition is as follows. Suppose the default outcome-i.e., the outcome if renegotiations fail-gives the buyer a higher value than the efficient service. If the buyer expects the service to be efficiently renegotiated, then he also expects a reduction in the trade price. This, in turn, makes him willing to accept the efficient service for a price reduction which is lower than his reduction in value in order to avoid a loss in the price dimension if renegotiations fail. With the bilateral monopoly structure ex post, the buyer in this sense is exposed to opportunism by the seller. Thus, for a given long-term contract, the buyer prefers ex ante that renegotiations do not take place in these situations. These expectations are credible, however, only if the buyer is sufficiently loss averse. With regard to sales contracts, we show that efficient renegotiations are more likely if the buyer is only mildly loss averse and if the environment is fairly uncertain. The latter finding is in line with Kőszegi and Rabin (2007), who show that being exposed to background risk makes a loss-averse decision maker less risk averse.

The ex ante optimal contract maximizes the expected surplus of the two parties including the buyer's expected losses. We show that the optimal long-term contract typically is an employment contract that gives a sufficiently high degree of discretion (in form of a large acceptance set) to the party that is designated to choose. The advantage of an employment contract with much discretion compared to contracts with little discretion, a sales contract in particular, is that it leads to less variations in the default outcome and thus also less variations in the ex post outcome. We thus provide a rationale for employment contracts in the absence of non-contractible investments.

Whether a buyer or a seller employment contract is optimal depends on the precise nature of the achievable costs and benefits. Suppose the gains from trading the buyer's preferred good are relatively high, while the gains from trading the seller's preferred good are relatively low. As outlined before, a highly loss-averse buyer might prefer to expect renegotiations not to take place. This is attractive under a buyer employment contract but less so under a seller employment contract where the losses in material gains from trade are severe. As a consequence, a buyer employment contract with much discretion results in frequent trade of a service of inefficiently high quality. Under a seller employment contract, the default outcome leads to very low material gains from trade and thus expecting renegotiations not to take place might not be a credible plan for the buyer. In this scenario, an employment contract that gives the seller a lot of discretion is uniquely optimal. Note that renegotiations here require that the price increases because the default service minimizes the seller's cost. Conventional wisdom, however, seems to suggest that under loss aversion prices should be rather sticky because customers should severely suffer from losses in the money dimension in form of price increases. We show that this reasoning is incorrect if the customers are expectation-based loss averse and rationally anticipate that the price will increase. Thus, loss aversion can accommodate the observation that prices often increase after renegotiations-e.g., the ultimate bill of a craftsman often being 
higher than the initially specified price.

In a second step, we extend our model by allowing the buyer to make a relationship-specific and non-contractible investment in the sense of Hart and Moore (1988). In our benchmark model the parties write a long-term contract because there is a change in the buyer's bargaining position. With relationship-specific investments a long-term contract is needed in order to protect the buyer's sunk investment against ex post opportunism by the seller. We show that loss aversion can reduce the hold-up problem, i.e., investment incentives can be increasing in the degree of loss aversion. This result is not due to the fact that loss aversion makes renegotiations harder. In fact, it occurs in cases where efficient renegotiation takes place. The loss-averse buyer is exploited by the seller whenever the original long-term contract is renegotiated ex post. This exploitation, however, is reduced if the buyer undertakes the investment. This explains why investment incentives can be increasing in the degree of loss aversion. If the buyer is sufficiently loss averse, however, not undertaking the investment can become a commitment for the buyer not to renegotiate the contract ex post, which protects him from ex post opportunism by the seller. This, in turn, leads to lower investment incentives with a loss-averse buyer.

The remainder of the paper is organized as follows. Before introducing the model in Section 2, we briefly discuss the related literature. The model is solved in Section 3. We start by analyzing spot contracting in Subsection 3.1. In order to analyze the outcome of renegotiations, we first describe the default outcome in Subsection 3.2 and thereafter-in Subsection 3.3-we characterize some general properties of a SP-PE. The outcome of renegotiations for sales contracts and employment contracts are characterized in Subsections 3.4 to 3.6. Optimal long-term contracts are analyzed in Subsection 3.7. Non-contractible investments and the arising hold-up problem are considered in Section 4. The final Section 5 summarizes our main findings and critically discusses some of the simplifying assumptions we impose. All proofs are relegated to Appendix A but proofs of purely technical results are relegated to Appendix B. Appendix C provides a precise formal definition of our equilibrium concept.

Related literature.-The theory of the firm and the literature on incomplete contracts goes back to Coase (1937), with the first formal model being found in Simon (1951). The modern game theoretic approaches, beginning with Grout (1984), abstract from ex post inefficiencies and focus on the ex ante inefficiencies caused by the hold-up problem, as introduced by Klein et al. (1978). ${ }^{6}$ One important strand of this modern literature investigates how property rights can be used to allocate the bargaining power ex post and thereby to enhance investment incentives (Grossman and Hart, 1986; Hart and Moore, 1990). If specific performance contracts are feasible, option contracts are an alternative to the allocation of property rights in order to restore investment incentives (Nöldeke and Schmidt, 1995). As pointed out by Hart and Moore (2008, p.2) "the emphasis on noncontractible ex ante investments seems overplayed" in this literature. In our model the achieved surplus of the different types of long-term contracts differ even in the

${ }^{6}$ Transaction costs as introduced and discussed by Williamson $(1975,1979,1985)$ are another source of inefficiencies caused by incomplete contracts. 
absence of non-contractible investments.

The seminal contribution by Hart and Moore (2008) posits that a contract provides a reference point for the parties' feelings of entitlements ex post. ${ }^{7}$ A party who feels shortchanged (relative to what she feels entitled to given the possible outcomes permitted by the contract) shades on performance, which leads to an ex post inefficiency. Hart and Moore focus on the trade-off arising between contractual rigidity and flexibility. Compared to a flexible contract, a rigid contract reduces the parties' desire to shade by leaving little room for disagreement over which party is entitled to what share of the rents. In contrast to a flexible contract, however, a rigid contract does not allow for an adjustment of contractual terms in the light of new information. This theory is then used by Hart (2009) to shed new light on the optimal allocation of ownership rights and indexing contracts, by Hart and Holmstrom (2010) to investigate the boundaries of the firm, and by Hart (2013) to reconsider non-contractible investments and the arising hold-up problem. ${ }^{8}$ All the aforementioned papers do not analyze contract renegotiation, which is at the heart of our analysis. The Hart-Moore approach is extended by Halonen-Akatwijuka and Hart (2013) in order to allow for renegotiation. They discuss examples under which the Hart-Moore approach can accommodate why parties leave contracts deliberately incomplete.

The Hart-Moore approach is based on several behavioral assumptions. Next to reference dependent preferences the parties have a self-serving bias and feel entitled to the best possible outcome the contract in place allows for. Moreover, the parties behave reciprocally in the sense that they can reduce their respective feelings of aggrievement by reducing the other party's utility from trade. ${ }^{9}$ While both our model as well as Hart and Moore's model create scope for ex post inefficiencies, in our model this ex post inefficiency is rooted purely in the expectationbased loss aversion of the buyer without any notion of self-serving bias or social preferences.

The paper closest related to our work is Herweg and Schmidt (2013). ${ }^{10}$ Both papers consider specific performance contracts and analyze how loss aversion affects the outcome of renegotiations ex post. While Herweg and Schmidt posit that the reference point at the renegotiation stage directly corresponds to the default outcome determined by the initial contract, we posit that it is determined by rational expectations formed ex ante and thus is affected by the initial contract only indirectly. Thus, both papers take an extreme - but complementary_-view regarding how the long-term contract shapes the reference point. The main focus of Herweg and Schmidt is on ex post inefficiencies caused by loss aversion. They show that loss aversion makes the renegotiated outcome sticky and inefficient, i.e., the delivered service and the price are insufficiently, if at all, adjusted to the realized state of nature. Furthermore, Herweg and Schmidt explore the implications of their theory for optimal long-term contracting and the allocation of ownership

\footnotetext{
${ }^{7}$ See also Hart and Moore (2007).

${ }^{8} \mathrm{~A}$ similar approach is used by Mori (2012) in order to explain why an authority relationship is more efficient than a contract in the presence of ex post adaption problems.

${ }^{9}$ Laboratory evidence for these assumptions is provided by Fehr et al. (2011a). See also Fehr et al. (2009), Fehr et al. (2011b), and Hoppe and Schmitz (2011).

${ }^{10}$ The experimental findings obtained by Bartling and Schmidt (2013) are in line with the predictions made by Herweg and Schmidt (2013).
} 
rights. The focus of our study is how expectations affect the parties' willingness to renegotiate and the outcome of renegotiations, which is not an issue in Herweg and Schmidt.

Finally, the paper is related to the recent and growing literature dealing with expectationbased loss aversion. Evidence for reference points being (at least partially) shaped by expectations is found in both laboratory data (Abeler et al., 2011; Ericson and Fuster, 2011; Gill and Prowse, 2012; Karle et al., 2012; Banerji and Gupta, 2014) as well as field data (Crawford and Meng, 2011; Bartling et al., 2013). Beginning with Heidhues and Kőszegi (2008), expectationbased loss aversion à la Köszegi and Rabin $(2006,2007)$ is applied to models of industrial organization (Heidhues and Kőszegi, 2014; Herweg and Mierendorff, 2013; Karle and Peitz, forthcoming; Karle, 2013; Rosato, 2013), contract design (Herweg et al., 2010; Macera, 2011; Daido and Murooka, 2013; Daido et al., 2013), mechanism design (Eisenhuth, 2012; Hahn et al., 2012), and inventory management (Herweg, 2013).

\section{THE MODEL}

\subsection{Trading Environment}

We consider an incomplete contracting environment similar to Bolton and Dewatripont (2005). A buyer (he) requires a service which can be provided by one of two sellers (she). The nature of the service will be commonly known when trade takes place but is unknown to the parties ex ante when they may write a long-term contract. There are $n$ kinds of the service that each seller can deliver, $x \in\left\{x_{1}, x_{2}, \ldots, x_{n}\right\} \equiv \mathcal{X}$ and there are $n$ equiprobable states of the world, $\theta \in\left\{\theta_{1}, \ldots, \theta_{n}\right\} \equiv \Theta$. We assume that $n \geq 3$ is odd. The buyer's benefit and a seller's cost from service $x \in \mathcal{X}$ being traded in state $\theta \in \Theta$ is denoted by $v(x, \theta)$ and $c(x, \theta)$, respectively. Trade of service $x$ in state $\theta$ can result in three different benefit-cost combinations,

$$
(v(x, \theta), c(x, \theta)) \in\left\{\left(v_{0}, c_{0}\right),\left(v_{L}, c_{L}\right),\left(v_{H}, c_{H}\right)\right\},
$$

where

$$
0 \equiv v_{0}<v_{L}<v_{H}, \quad 0 \equiv c_{0}<c_{L}<c_{H}, \quad 0<v_{H}-c_{H}<v_{L}-c_{L}
$$

Trading the good that leads to the low-value/low-cost outcome maximizes the material gains from trade ex post. Henceforth, the outcome $\left(v_{L}, c_{L}\right)$ will be called "materially efficient". In state $\theta_{i} \in \Theta$ service $x_{i}$ is the unique service that results in the materially efficient outcome. Furthermore, in any state $\theta_{i} \in \Theta$ there exist services $x_{j}$ and $x_{k}$ different from $x_{i}$ that result in the high-value outcome and the worthless outcome, respectively. Formally, for each $\theta_{i} \in \Theta$, $v\left(x, \theta_{i}\right)=v_{L}$ if and only if $x=x_{i}, v\left(x_{j}, \theta_{i}\right)=v_{H}$ for some $x_{j} \neq x_{i}$, and $v\left(x_{k}, \theta_{i}\right)=0$ for some $x_{k} \neq x_{i}, x_{j}$. Finally, ex ante each service $x \in \mathcal{X}$ is equally likely to result in the high-value outcome or the worthless outcome. ${ }^{11}$

\footnotetext{
${ }^{11}$ The symmetry assumption regarding materially inefficient outcomes and the assumption of a unique materially efficient service are merely imposed in order to simplify the exposition. Any of these assumptions can be relaxed, which would also allow for considering an even number of states.
} 
In order to reduce the number of cases that need to be considered, we assume the following:

Assumption 1. $v_{L}>\max \left\{2 c_{L}, v_{H} / 2\right\}$

\subsection{Contracts, Renegotiation, and the Sequence of Events}

Over the lifetime of the trading relationship, sellers can make take-it-or-leave-it offers to the buyer and the buyer can at any time be involved in at most one contractual relationship. At date 0 , sellers simultaneously and non-cooperatively make contract offers to the buyer. The state of the world is not verifiable and thus cannot be contracted upon. ${ }^{12}$ A long-term contract offer $C_{E, \mathcal{A}}(\bar{p})$ specifies an acceptance set $\mathcal{A} \subseteq \mathcal{X}$ from which party $E \in\{B, S\}$ is designated to freely choose service $x \in \mathcal{A}$ to be delivered at price $\bar{p} \in \mathbb{R}$. This contractual arrangement thus represents a buyer employment contract for $E=B$, and a seller employment contract for $E=S$. If $\mathcal{A}$ is a singleton, the offered contract is a simple sales contract specifying a particular service to be delivered at a pre-specified price.

At date 1, upon receiving both sellers' offers, the buyer decides whether to accept one of these offers or to reject them all. In the case of rejection, which we denote by $C=\varnothing$, contracting is deferred to date 4 . In order to make this decision, the buyer forms rational expectations about the value of the service he will ultimately consume and about the price he has to pay for it. For a loss-averse buyer these rational expectations formed at date 1 shape a reference point. The reference point affects the buyer's evaluation of renegotiation or spot contracting at date 4 . We will explain this in more detail below.

At date 2, upon observing the buyer's decision, each seller has to decide whether she stays in the market or pursues a fleeting outside option, which is no longer available after date 2 . If a seller chooses to leave the market, she obtains $\pi>0$ but close to zero. After date 2, each seller's outside option equals zero. A seller whose offer was accepted by the buyer at date 1 cannot leave the market anymore because she is committed to the trading relationship. Sellers make the decision whether to leave the market simultaneously and non-cooperatively. Afterward, each seller observes how many sellers remain in the market.

At date 3, the state of the world, $\theta \in \Theta$, materializes and is observed by all parties. At date 4 , if the buyer accepted one of the initial contract offers, renegotiation takes place with the respective seller offering a renegotiated contract $\left(x^{R}, p^{R}\right) \in \mathcal{X} \times \mathbb{R}$ to the buyer. If the buyer rejected all the original contract offers, each seller who did not leave the market makes a take-it-or-leave-it spot contract offer $\left(x^{\text {spot }}, p^{\text {spot }}\right) \in \mathcal{X} \times \mathbb{R}$.

If the buyer signed a long-term contract, at date 5, he decides whether to accept the seller's renegotiation offer. Otherwise, the buyer decides which (if any) of the spot contract offers to accept. Finally, at date 6 the service of the concluded contract at date 5 is delivered at the specified price and costs and benefits are realized. If the buyer rejected all contract offers made

\footnotetext{
${ }^{12}$ We presume that the parties cannot use a third party to make the state verifiable as in Maskin (1999).
} 


\begin{tabular}{|c|c|c|c|c|c|}
\hline 0 & 1 & 2 & 3 & 4 & 5 \\
\hline $\begin{array}{l}\text { Sellers } \\
\text { make } \\
\text { initial } \\
\text { contract } \\
\text { offers }\end{array}$ & $\begin{array}{l}\text { Buyer } \\
\text { decides on } \\
\text { acceptance } \\
\text { and forms } \\
\text { expectations }\end{array}$ & $\begin{array}{l}\text { Sellers } \\
\text { decide } \\
\text { whether } \\
\text { to stay in } \\
\text { the market }\end{array}$ & $\begin{array}{l}\text { Nature } \\
\text { determines } \\
\text { the state of } \\
\text { the world }\end{array}$ & $\begin{array}{l}\text { Renegotition } \\
\text { or spot } \\
\text { contracting }\end{array}$ & $\begin{array}{l}\text { Buyer } \\
\text { decides on } \\
\text { acceptance, } \\
\text { payoffs are } \\
\text { realized }\end{array}$ \\
\hline
\end{tabular}

Figure 1: Sequence of events

at date 1 and date 4, all parties receive a material payoff of zero but sellers who left the market at date $2 .{ }^{13,14}$

Regarding the buyer's decisions at date 1 and at date 4, we assume the following tie-breaking rules: If the buyer is indifferent between accepting an offer and rejecting one or more offers, the buyer accepts the offer. When being indifferent which contract offer to accept, the buyer picks one offer at random with equal probability. ${ }^{15}$ Throughout the analysis we focus on equilibria in pure strategies. The sequence of events is summarized in Figure 1.

\subsection{Buyer's Preferences and Seller's Profit}

The buyer is expectation-based loss averse according to Kőszegi and Rabin $(2006,2007)$ and his utility has two components: material utility and loss utility. Material utility from consuming $x$ in state $\theta$ at price $p$ is $v(x, \theta)-p$. Loss utility is derived by comparing the outcome in a particular dimension, "value" or "money", to its respective reference level. The reference point for a given dimension, which is determined by rational expectations formed at the end of date 1 , is independent across the two dimensions and typically stochastic. At date 4, the buyer takes these expectations as given and both the renegotiation or spot contract offer are evaluated in comparison to this reference point, where losses are evaluated separately in both dimensions. Specifically, when the buyer accepts a long-term contract offer $C_{E, \mathcal{A}}(\bar{p})$ at date 1 , he forms expectations regarding the outcome of renegotiation with the seller whose contract he signed. With our focus on pure strategies, the buyer's expectations under long-term contract $C_{E, \mathcal{A}}(\bar{p})$ about the outcome and the trade price implemented at date 5 if state $\theta \in \Theta$ is realized comprise

\footnotetext{
${ }^{13}$ Regarding the time elapsed between two different points in time we have the following in mind. First the parties meet and may write a long term contract (date 0 - date 2). This happens all within a short period of time. Thereafter, between date 2 and date 3 quite some time elapses. After the state of the world has been materialized, the parties renegotiate the contract (date 3 - date 5), which again happens within a relative short period.

${ }^{14}$ The assumption that the buyer does not update his reference point after observing the state of nature at date 3 allows us to sidestep the issue of paper losses in the spirit of Köszegi and Rabin (2009). In Herweg and Schmidt (2013) the parties form their reference point after observing the materialized state of nature.

${ }^{15}$ Regarding spot contracting we impose an additional tie-breaking rule in order to simplify some proofs. If the buyer is indifferent between two distinct offers both of which he would rather accept than reject, the buyer chooses the offer involving the higher value.
} 
a single value-cost pair

$$
\left(\hat{v}\left(\theta, C_{E, \mathcal{A}}(\bar{p})\right), \hat{p}\left(\theta, C_{E, \mathcal{A}}(\bar{p})\right)\right)
$$

Define

$$
\Lambda\left(C_{E, \mathcal{A}}(\bar{p})\right) \equiv\left\{\left(\hat{v}\left(\theta, C_{E, \mathcal{A}}(\bar{p})\right), \hat{p}\left(\theta, C_{E, \mathcal{A}}(\bar{p})\right)\right)\right\}_{\theta \in \Theta}
$$

as buyer's set of expectations, comprising of $n$ value-price pairs, regarding the outcome of renegotiations. If the buyer rejects all the contract offers at date $1, C=\varnothing$, he forms expectations about the outcome that spot contracting will take. With sellers observing the state of the world at the time of their spot contract offers, the buyer expects the sellers' offers to depend on the state of the world. The buyer expects spot contracting in state $\theta \in \Theta$ to result in a particular value to be delivered at a particular price,

$$
(\hat{v}(\theta, \varnothing), \hat{p}(\theta, \varnothing))
$$

Note that the buyer expecting to reject all spot contract offers in state $\theta \in \Theta$ corresponds to $(\hat{v}(\theta, \varnothing), \hat{p}(\theta, \varnothing))=(0,0)$. The buyer's complete set of expectations regarding spot contracting is denoted by

$$
\Lambda(\varnothing) \equiv\{(\hat{v}(\theta, \varnothing), \hat{p}(\theta, \varnothing))\}_{\theta \in \Theta}
$$

For given expectations $\Lambda(C)$, with $C \in\left\{C_{E, \mathcal{A}}(\bar{p}), \varnothing\right\}$, the buyer's overall utility at date 5 if the seller delivers a service resulting in value $v$ at price $p$ is

$$
\begin{aligned}
& U(v, p \mid \Lambda(C)) \\
& \quad=v-p-\frac{1}{n} \cdot \lambda \cdot\left\{\sum_{\{\theta \in \Theta \mid \hat{v}(\theta, C)>v\}}[\hat{v}(\theta, C)-v]+\sum_{\{\theta \in \Theta \mid \hat{p}(\theta, C)<p\}}[p-\hat{p}(\theta, C)]\right\} .
\end{aligned}
$$

For simplicity, we abstract from any gain utility and the weight on losses is $\lambda \geq 0 .{ }^{16}$ Here, $\lambda$ captures both the buyer's degree of reference dependence and his magnitude of loss aversion. Following the literature, we assume that the weight the buyer places on loss utility does not exceed the weight placed on material utility (Herweg et al., 2010).

Assumption 2 (no dominance of loss utility). $\quad \lambda \leq 1$

In contrast to the buyer, the sellers are risk and loss neutral. At date 5, a seller's profit from delivering a service which $\operatorname{costs} c$ at price $p$ is

$$
\Pi(c, p)=p-c
$$

\footnotetext{
${ }^{16}$ This assumption—which is also imposed by de Meza and Webb (2007) and Herweg and Mierendorff (2013) has no qualitative effects on our results.
} 
Equilibrium concept.-If the buyer is not loss averse, we apply the standard notion of subgame perfect equilibrium in pure strategies. For a loss-averse buyer we augment the concept of subgame perfect equilibrium by incorporating that the buyer's behavior has to be a personal equilibrium (PE) as defined in Kőszegi and Rabin (2006). The PE requires that the buyer's expectations formed at date 1 (initial contracting) are such that his behavior at date 5 (renegotiation or spot contracting) is consistent with his lagged expectations. At date 5 the buyer simply selects the option among the available ones that maximizes his utility for the given reference point formed at date 1 . The available options at date 5 are dependent on the sellers' behavior. In equilibrium sellers correctly anticipate the buyer's reference point, i.e., the offer of each seller at date 4 takes the buyer's reference point formed at date 1 into account. At date 1, when forming his expectations, the buyer correctly anticipates that the sellers will optimally react on this reference point at date 4. Moreover, PE embodies the consistency criterion that the buyer can only form plans that he will follow through. We will call an equilibrium of the type described above subgame perfect personal equilibrium (SP-PE). Due to the self-fulfilling prophecy nature of the $\mathrm{PE}$ concept, there are typically multiple PE, which in turn implies that there are often multiple SP-PE. In this case, at date 1 the buyer is assumed to choose the plan among all consistent plans that gives him the highest expected utility, i.e., the preferred personal equilibrium (PPE) in the language of Kőszegi and Rabin (2006). We call the corresponding equilibrium in which sellers correctly anticipate this behavior of the buyer as subgame perfect preferred personal equilibrium (SP-PPE). A precise definition of our equilibrium concept is provided in the Appendix C.

\subsection{Benchmark Case without Loss Aversion}

As a benchmark, consider the case where the buyer is not loss averse and his behavior is solely determined by material considerations. First, assume the buyer rejected all contract offers at date 1 and thus spot contracting takes place at date 4 . If both sellers are active at date 4 , then there is Bertrand competition which is associated with zero profits for both sellers. With each seller's outside option being strictly positive at date 2 , two sellers staying in the market after date 2 is incompatible with pure-strategy subgame perfection. Hence, only a single seller is active on the spot market. This seller will charge a price that makes the buyer just indifferent between accepting and rejecting the offer, i.e., $p^{\text {spot }}=v^{\text {spot }}$. Furthermore, the seller will propose the service that leads to the highest gains from trade, i.e., $v^{\text {spot }}=v_{L}$.

Now, suppose the buyer accepted some seller's long-term contract $C_{E, \mathcal{A}}(\bar{p})$ at date 1 . At date 4 , for any realized state $\theta_{i}$, the parties will always agree upon trading the materially efficient good $x_{i}$. The initial contract only determines the parties outside option and therefore the necessary adjustment of the price. In other words, the precise structure of the long-term contract is irrelevant. With both sellers being active at date 1, the sellers compete with their long-term contract offers for the buyer in a Bertrand fashion, i.e., each seller makes the best feasible contract 
offer to the buyer that allows her to obtain a profit equal to her fleeting outside option.

Observation 1. Suppose the buyer is not loss averse. Both sellers offer long-term contracts that result in profits equal to $\pi$ and will be accepted by the buyer with probability 1/2. The long-term contract offers can take any form, e.g., sales contract, buyer employment contract, and seller employment contract. The buyer's expected utility is $v_{L}-c_{L}-\pi$.

Our model presumes that there is perfect competition between sellers at date 1 and that competition is significantly reduced at date 4 . In fact we assume that there is a bilateral monopoly and that the seller has all the bargaining power at date 4 . A long-term contract protects the buyer against being exploited by a monopolist at date 4 , and therefore is observed in all subgameperfect equilibria.

\section{THE ANALYSis}

We start by analyzing the spot contracting subgame. Before analyzing the renegotiation subgame and long-term contracting, we introduce some notation and some preliminary results. The analysis, thereafter, is conducted separartely for different sizes of the acceptance set.

\subsection{Spot Contracting}

If the buyer rejects all long-term contract offers at date 1, only one seller will stay in the market after date 2. As we will show below, the seller staying in the market makes a profit that exceeds her fleeting outside option $\pi>0$.

First, note that the buyer expecting the outcome of spot contracting to depend on the state of the world is incompatible with SP-PE. In other words, in any SP-PE we have $(\hat{v}(\theta, \varnothing), \hat{p}(\theta, \varnothing))=$ $\left(v^{\text {spot }}, p^{\text {spot}}\right)$ for all $\theta \in \Theta$. Intuitively, with the buyer's expectations being fixed from date 1 onward, all states of the world are ex post identical and (generically) the seller is harmed from doing different things in different states.

Now, suppose the buyer expects to purchase a service resulting in value $v^{\text {spot }} \in\left\{v_{L}, v_{H}\right\}$ at price $p^{\text {spot }}$. The buyer's utility from rejecting the seller's spot contract offer is $-\lambda v^{\text {spot }}$. Thus, if the seller offers to deliver a service resulting in value $v^{\text {spot }}$ at a price $p \geq p^{\text {spot }}$, the buyer is still willing to accept this offer as long as $v^{\text {spot }}-p-\lambda\left(p-p^{\text {spot }}\right) \geq-\lambda v^{\text {spot }}$. With the seller charging the highest acceptable price and with expectations being met in equilibrium, $p^{\text {spot }}=(1+\lambda) v^{\text {spot }}$ is the only price consistent with the buyer expecting to purchase a service resulting in value $v^{\text {spot }}$. The buyer's resulting utility is $-\lambda v^{\text {spot }}$.

Consider the case where the buyer expects to obtain value $v_{L}$, i.e. $\left(v^{\text {spot }}, p^{\text {spot }}\right)=\left(v_{L},(1+\right.$ $\left.\lambda) v_{L}\right)$. Obviously, the seller cannot benefit from a deviation to offering a worthless service instead, which would be accepted by the buyer only for a negative price. While the buyer would accept a high-value service at price $p \geq p^{\text {spot }}$ as long as $v_{H}-p-\lambda\left(p-p^{\text {spot }}\right) \geq-\lambda v_{L}$, this 
deviation is not profitable for the seller even for the highest price still accepted by the buyer, $p=\left[v_{H}+\lambda(2+\lambda) v_{L}\right] /(1+\lambda)$.

Finally, note that outcome $\left(v^{\text {spot }}, p^{\text {spot }}\right)=(0,0)$ is incompatible with equilibrium. In this case the seller could profitably deviate by offering delivery of the materially efficient service at price $p=v_{L} /(1+\lambda)$. At this price the buyer is just indifferent between accepting and rejecting the seller's contract offer and the seller makes a strictly positive profit, $v_{L} /(1+\lambda)-c_{L}$, that exceeds her outside option.

Hence, a spot-contracting equilibrium with $\left(v^{\text {spot }}, p^{\text {spot }}\right)=\left(v_{L},(1+\lambda) v_{L}\right)$ always exists. The buyer's utility in this equilibrium is higher than in a possibly existing equilibrium in which he expects to obtain a high-value service. ${ }^{17}$

Proposition 1. Suppose the buyer rejected all contract offers at date 1. The spot contracting SP-PPE consists of $\Lambda(\varnothing)=\left\{\left(v_{L},(1+\lambda) v_{L}\right)\right\}_{\theta \in \Theta}$ and only one seller staying in the market.

\subsection{Further Notation-Default Outcome}

In order to characterize the outcome of renegotiation, it is important to know what the outcome is if renegotiations fail. Let

$$
\left(v^{D}(\theta, C), c^{D}(\theta, C)\right)
$$

denote the "default" value-cost pair that will be implemented under contract $C=C_{E, \mathcal{A}}(\bar{p})$ if renegotiation breaks down (or is not offered in the first place) after state $\theta \in \Theta$ is realized. While for $E=S$ the seller will choose a service resulting in the minimum cost feasible given set $\mathcal{A}$, for $E=B$ the buyer will opt for a service that results in the highest possible value given set $\mathcal{A}$. Let

$$
\Theta_{k}(E, \mathcal{A}) \equiv\left\{\theta \in \Theta \mid\left(v^{D}(\theta, C), c^{D}(\theta, C)\right)=\left(v_{k}, c_{k}\right)\right\}
$$

represent the set of all states that are associated with default outcome $\left(v_{k}, c_{k}\right)$, where $k \in$ $\{0, L, H\}$, given that party $E \in\{B, S\}$ is designated to choose from set $\mathcal{A}$. Letting

$$
Q_{k}(E, \mathcal{A})=\operatorname{Pr}\left(\theta \in \Theta_{k}(E, \mathcal{A})\right)=\frac{\left|\Theta_{k}(E, \mathcal{A})\right|}{n}
$$

denote the ex ante probability that contract $C_{E, \mathcal{A}}(\bar{p})$ results in default outcome $\left(v_{k}, c_{k}\right)$, we have

$$
\begin{aligned}
& Q_{H}(B, \mathcal{A})=Q_{0}(S, \mathcal{A})= \begin{cases}1 & \text { if }|\mathcal{A}|>\frac{n+1}{2} \\
1-\frac{s+1}{n} & \text { if }|\mathcal{A}|=\frac{n+1}{2}-s \text { for } s \in\left\{0, \ldots, \frac{n-1}{2}\right\}\end{cases} \\
& Q_{L}(B, \mathcal{A})=Q_{L}(S, \mathcal{A})= \begin{cases}0 & \text { if }|\mathcal{A}|>\frac{n+1}{2} \\
\frac{1}{n} & \text { if }|\mathcal{A}| \leq \frac{n+1}{2}\end{cases}
\end{aligned}
$$

\footnotetext{
${ }^{17}$ A spot-contracting equilibrium with $v^{\text {spot }}=v_{H}$ and $p^{\text {spot }}=(1+\lambda) v_{H}$ exists if $\lambda \geq\left[\left(c_{H}-c_{L}\right) /\left(v_{H}-v_{L}\right)\right]-1$. Assuming that spot contracting always leads to trade of the materially efficient service has no impact on the long-term contracts offered at date 1 in a SP-PE.
} 
and

$$
Q_{0}(B, \mathcal{A})=Q_{H}(S, \mathcal{A})= \begin{cases}0 & \text { if }|\mathcal{A}| \geq \frac{n+1}{2} \\ \frac{s}{n} & \text { if }|\mathcal{A}|=\frac{n+1}{2}-s \text { for } s \in\left\{1, \ldots, \frac{n-1}{2}\right\}\end{cases}
$$

We refer to acceptance sets of size $|\mathcal{A}|>(n+1) / 2$, which contain both the high-value and the worthless outcome for sure, as large. An acceptance set of seize $|\mathcal{A}|=(n+1) / 2$, which guarantees the buyer a default value of at least $v_{L}$ and the seller a default cost of at most $c_{L}$, is referred to as medium. Acceptance sets of size $|\mathcal{A}|<(n+1) / 2$ are referred to as small.

\subsection{Preliminary Analysis}

We begin with some basic observations regarding the buyer's expectations concerning renegotiation when he accepted a long-term contract $C=C_{E, \mathcal{A}}(\bar{p})$ offered at date 0 . The buyer expecting renegotiations not to occur if state $\theta \in \Theta$ has been realized corresponds to $(\hat{v}(\theta, C), \hat{p}(\theta, C))=$ $\left(v^{D}(\theta, C), \bar{p}\right)$.

Lemma 1. Generically, in a $S P-P E$, for all $\theta^{\prime}, \theta^{\prime \prime} \in \Theta$ with $\theta^{\prime} \neq \theta^{\prime \prime}$, if $v^{D}\left(\theta^{\prime}, C\right)=v^{D}\left(\theta^{\prime \prime}, C\right)$, then $\left(\hat{v}\left(\theta^{\prime}, C\right), \hat{p}\left(\theta^{\prime}, C\right)\right)=\left(\hat{v}\left(\theta^{\prime \prime}, C\right), \hat{p}\left(\theta^{\prime \prime}, C\right)\right)$.

According to Lemma 1, the buyer's expectations regarding the outcome of renegotiations in state $\theta \in \Theta$ is fully determined by this state's default outcome. Intuitively, from (7) it follows that the buyer's utility from obtaining value $v$ at price $p$ does not depend on the state of the world per se. Therefore, when contemplating whether to accept or reject the seller's renegotiation offer, only the buyer's default outcome matters but not the state of the world in which this default outcome is brought about. Therefore, (generically) the seller is harmed by making different renegotiation offers in different states with the same default outcome, which is anticipated by the buyer.

To state the following observations concisely, let $\left(\hat{v}_{k}, \hat{p}_{k}\right)$ (with $k \in\{0, L, H\}$ ) denote the buyer's expectations regarding renegotiations for state $\theta \in \Theta$ with default outcome $v^{D}(\theta, C)=$ $v_{k}$. A first observation is that if renegotiation occurs for default outcome $v_{k}$, then the seller's renegotiation offer makes the buyer just indifferent between the default outcome at price $\bar{p}$ and the renegotiated outcome at price $\hat{p}_{k}$. Next, whenever the buyer expects renegotiations to result in a higher value than the default outcome, $\hat{v}_{k}>v_{k}$, he has to expect to pay a markup $\hat{p}_{k}-\bar{p}>0$-facing a strict increase in cost, the seller would never offer a price below the initial price $\bar{p}$. Finally, if the buyer expects renegotiation to result in a lower value than the initial contract, $\hat{v}_{k}<v_{k}$, he has to expect a price reduction $\hat{p}_{k}<\bar{p}$-when offered lower value at a higher price, the buyer himself would be strictly better off by rejecting renegotiation.

The further analysis of the outcome of renegotiations can involve numerous case-by-case analyses. For example, a small acceptance set (e.g., a sales contract) allows for three different default outcomes such that the buyer's beliefs are represented by a triplet of value-price pairs, 
$\Lambda(C)=\left\{\left(\hat{v}_{0}, \hat{p}_{0}\right),\left(\hat{v}_{L}, \hat{p}_{L}\right),\left(\hat{v}_{H}, \hat{p}_{H}\right)\right\}$. With three possible outcomes to renegotiate to, in principle the buyer might expect $3^{3}=27$ different outcomes of renegotiation. The following lemmas are helpful in order to reduce the number of cases that need to be distinguished.

First, we can rule out that the worthless service is traded ex post. This is due to Assumptions 1 and 2, which ensure that the gains from materially efficient trade are sufficiently large compared to the potential losses.

Lemma 2. In any $S P-P E, \hat{v}_{0}>0$.

While Lemma 2 allows us to narrow down the form a SP-PE may take, the logic underlying its proof allows to draw also important off-equilibrium path implications.

Corollary 1. Deviating from the buyer's expectations by offering renegotiations to a worthless service is never profitable for the seller.

With the increase in value being higher when moving from a worthless service to the lowvalue service than when moving from the low-value service to a high-value service, we obtain the following result regarding the renegotiated prices.

Lemma 3. In any $S P-P E$, if $\hat{v}_{0}=v_{L}$ and $\hat{v}_{L}=v_{H}$, then $\hat{p}_{L}<\hat{p}_{0}$.

Moreover, and most importantly, "criss-cross" renegotiation do not occur in equilibrium.

Lemma 4. Generically, in any SP-PE, $\hat{v}_{0} \leq \hat{v}_{L} \leq \hat{v}_{H}$.

Roughly spoken, if the buyer expects a lower-value (higher-value) default outcome $v_{k}$ to be renegotiated upward (downward) to a weakly higher (lower) value, then he cannot rationally expect a higher-value (lower-value) default outcome $v_{\tau}>v_{k}\left(v_{\tau}<v_{k}\right)$ to be renegotiated downward (upward) to a value strictly below (above) the renegotiation value he expects for the low-value (high-value) default outcome $v_{k}$. Figure 2(a) and (b) illustrate two examples of expectations that are ruled out by Lemma 4 for the case of a small acceptance set. Note, however, that Lemma 4 not only allows for "weakly monotonic" renegotiation as in Figure 2(c), but also for "non-monotonic" materially efficient renegotiation as depicted in Figure 2(d).

For future reference, define

$$
\tilde{\lambda}_{E, \mathcal{A}} \equiv \frac{1}{Q_{H}(E, \mathcal{A})}\left[\frac{c_{H}-c_{L}}{v_{H}-v_{L}}-1\right]
$$

which is an important threshold for the characterization of the SP-PPEa. Additionally, let $\Lambda^{*}\left(C_{E, \mathcal{A}}(\bar{p})\right)$ denote the buyer's equilibrium expectations regarding the outcome of renegotiation under long-term contract $C_{E, \mathcal{A}}(\bar{p})$. 
(a)

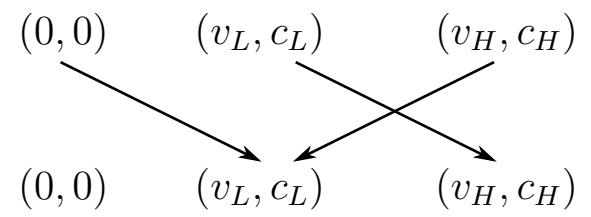

$(c)$

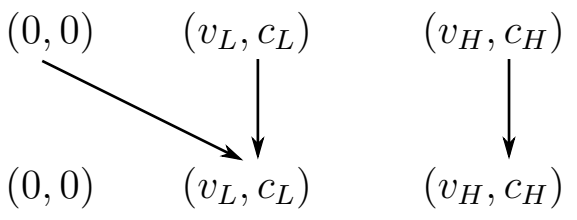

(b)

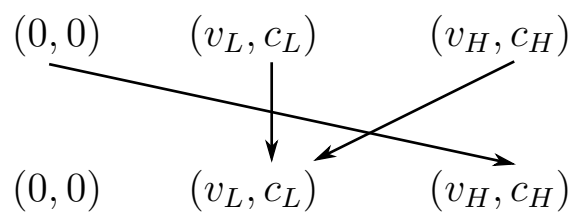

$(d)$

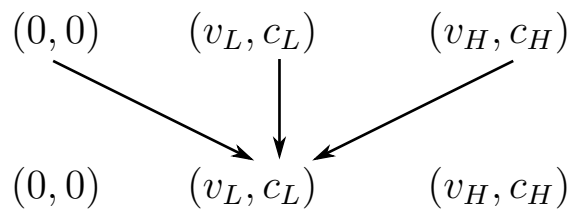

Figure 2: Illustration of Lemma 4 for $|\mathcal{A}| \leq(n+1) / 2$.

\subsection{Sales Contract / Employment Contracts with Small Acceptance Sets}

Suppose the buyer accepted a contract with price $\bar{p}$ and a small acceptance set, $|\mathcal{A}|<(n+1) / 2$, which encompasses the prominent case of a sales contract. Within this class of contracts any form of the default outcome may occur, $(0,0),\left(v_{L}, c_{L}\right)$ or $\left(v_{H}, c_{H}\right)$. Lemmas 2 and 4 leave us with four sets of expectations to consider, namely $\Lambda^{L L L}, \Lambda^{L L H}, \Lambda^{L H H}$, and $\Lambda^{H H H}$. First, as depicted in Figure 2(d), suppose the buyer expects renegotiation always to lead to provision of the materially efficient service, i.e.,

$$
\Lambda\left(C_{E, \mathcal{A}}(\bar{p})\right)=\left\{\left(v_{L}, \hat{p}_{0}^{L L L}\right),\left(v_{L}, \bar{p}\right),\left(v_{L}, \hat{p}_{H}^{L L L}\right)\right\}=: \Lambda^{L L L}
$$

Regarding the trade price, the buyer expects to obtain a discount in comparison to the price specified in the original contract if $v^{D}(\theta, C)=v_{H}$, whereas he expects to be charged a mark-up if $v^{D}(\theta, C)=0$. Formally, $\hat{p}_{H}^{L L L}<\bar{p}<\hat{p}_{0}^{L L L}$. With the seller having all the bargaining power at the renegotiation stage, the buyer's price expectations are pinned down by $U\left(v_{L}, \hat{p}_{0}^{L L L} \mid \Lambda^{L L L}\right)=$ $U\left(0, \bar{p} \mid \Lambda^{L L L}\right)$ and $U\left(v_{L}, \hat{p}_{H}^{L L L} \mid \Lambda^{L L L}\right)=U\left(v_{H}, \bar{p} \mid \Lambda^{L L L}\right)$ such that

$$
\hat{p}_{0}^{L L L}=\bar{p}+\frac{(1+\lambda) v_{L}}{1+\lambda\left(Q_{L}(E, \mathcal{A})+Q_{H}(E, \mathcal{A})\right)}
$$

and

$$
\hat{p}_{H}^{L L L}=\bar{p}-\frac{v_{H}-v_{L}}{1+\lambda Q_{H}(E, \mathcal{A})} .
$$

The prices consistent with materially efficient renegotiations allow for the important observation that loss aversion creates scope for the seller to exploit the buyer during renegotiations. First, due to the buyer's attachment to value $v_{L}$, the renegotiated mark-up in case of a worthless default outcome exceeds the buyer's actual increase in value, $\hat{p}_{0}^{L L L}-\bar{p}>v_{L}$. Likewise, expecting a price concession in case of a high-value default outcome makes the buyer attached to the idea of obtaining a discount. This, in turn, leads to the buyer accepting a price concession 
that falls short of the actual reduction on value, $\bar{p}-\hat{p}_{H}^{L L L}<v_{H}-v_{L}$. Note that both sorts of exploitation become more severe the higher the buyer's degree of loss aversion.

If the buyer expects renegotiations always to result in materially efficient trade, there is no scope for the seller to profitably deviate from the buyers expectations: any renegotiation offer different from those expected by the buyer either will be rejected by the buyer or is unprofitable for the seller to make in the first place. Moreover, expecting that renegotiations always lead to the implementation of the materially efficient outcome always is a consistent plan for the buyer. We can now state our first result regarding the subgame beginning with the choice of an employment contract with a small acceptance set.

Proposition 2. Consider $C=C_{E, \mathcal{A}}(\bar{p})$ with $|\mathcal{A}|<(n+1) / 2$. There always exists a SP-PE with $\Lambda^{*}(C)=\Lambda^{L L L}$.

According to Proposition 2, if the buyer expects that renegotiations are always materially efficient, the materially efficient service is indeed always delivered ex post-independent of the buyer's degree of loss aversion. In other words, if the parties expect that renegotiations are likely to take place, renegotiations will take place fairly often and the materially efficient outcome is always achieved. As we will show below, however, materially efficient renegotiations are not always the SP-PPE. The buyer's expected utility under materially efficient renegotiation is

$$
\begin{aligned}
E U\left(\Lambda^{L L L}\right)=v_{L}-\bar{p}-Q_{0}(E, \mathcal{A}) & (1+\lambda) v_{L} \\
& +Q_{H}(E, \mathcal{A}) \cdot \frac{1-\lambda\left(1-Q_{H}(E, \mathcal{A})\right)}{1+\lambda Q_{H}(E, \mathcal{A})} \cdot\left(v_{H}-v_{L}\right)
\end{aligned}
$$

Next, the buyer might expect the worthless default outcome to be renegotiated to the provision of the materially efficient service at a positive mark-up and renegotiations not to occur if the default outcome is of high value to him. Let these expectations be denoted by

$$
\Lambda\left(C_{E, \mathcal{A}}(\bar{p})\right)=\left\{\left(v_{L}, \hat{p}_{0}^{L L H}\right),\left(v_{L}, \bar{p}\right),\left(v_{H}, \bar{p}\right)\right\}=: \Lambda^{L L H},
$$

where $\bar{p}<\hat{p}_{0}^{L L H}$. The buyer's price expectations are pinned down by $U\left(v_{L}, \hat{p}_{0}^{L L L} \mid \Lambda^{L L H}\right)$ $=U\left(0, \bar{p} \mid \Lambda^{L L H}\right)$, which can be solved for

$$
\hat{p}_{0}^{L L H}=\bar{p}+\frac{1+\lambda}{1+\lambda Q_{H}(E, \mathcal{A})} v_{L} .
$$

Again, the mark-up in prices is higher than the actual increase in value, $\hat{p}_{0}^{L L H}-\bar{p} \geq v_{L}$, i.e., the buyer is exploited because of his attachment with regard to the value dimension. If $v^{D}(\theta, C)=$ $v_{H}$, however, the seller might possibly benefit from deviating from the buyer's expectations by offering provision of the materially efficient service at a discount price. This particular deviation may be profitable because it does not impose any losses in the money dimension on the buyer and leads to only a moderate loss in the value dimension such that the concession in the trade price necessary to make the buyer agree to renegotiation is not overly high . With 
$p^{R}=\bar{p}-\left(1+\lambda Q_{H}(E, \mathcal{A})\right)\left(v_{H}-v_{L}\right)$ being the maximum price at which the buyer is willing to accept renegotiation, i.e. $U\left(v_{L}, p^{R} \mid \Lambda^{L L H}\right)=U\left(v_{H}, \bar{p} \mid \Lambda^{L L H}\right)$, the seller refrains from offering renegotiation to the efficient service if $p^{R}-c_{L} \leq \bar{p}-c_{H}$. This, in turn, holds if and only if the buyer is fairly loss averse, i.e., $\lambda \geq \tilde{\lambda}_{E, \mathcal{A}}$ defined by (15). As it turns out, no other deviation is profitable for the seller such that expectations $\Lambda^{L L H}$ are part of a SP-PE if $\lambda \geq \tilde{\lambda}_{E, \mathcal{A}}$. The buyer's expected utility under expectations $\Lambda^{L L H}$ is

$$
\begin{aligned}
E U\left(\Lambda^{L L H}\right)=v_{L}-\bar{p}-Q_{0}(E, \mathcal{A}) & (1+\lambda) v_{L} \\
+ & Q_{H}(E, \mathcal{A}) \cdot\left[1-\lambda\left(1-Q_{H}(E, \mathcal{A})\right)\right] \cdot\left(v_{H}-v_{L}\right) .
\end{aligned}
$$

Regarding the two sets of expectations considered so far, what is the buyer's preferred plan consistent with subsequent equilibrium play? Comparison of (19) and (22) reveals that the buyer prefers less variation in prices, as embodied by expectations $\Lambda^{L L H}$, over stability in the value dimension at a low level, as embodied by expectations $\Lambda^{L L L}$, whenever the former expectations are consistent with subsequent rational behavior, i.e., whenever $\lambda \geq \tilde{\lambda}_{E, \mathcal{A}}$. Inspection of the prices consistent with these courses of renegotiation, cf. (18), (17) and (21), provides some intuition. For both $\Lambda^{L L L}$ and $\Lambda^{L L H}$ the buyer is exploited in case of a worthless default outcome because of his attachment to the idea of obtaining some valuable service. While this sort of exploitation is even stronger under $\Lambda^{L L H}$, the buyer is exploited under $\Lambda^{L L L}$ also in the case of a high-value default outcome because of his attachment to the idea of obtaining a price concession. Since the degree of exploitation increases in the buyer's degree of loss aversion, for a highly loss-averse buyer the expectation of being exploited on two accounts under $\Lambda^{L L L}$ (together with the fact that he will sometimes obtain the highest possible value under $\Lambda^{L L H}$ ) makes expecting materially efficient renegotiations very unattractive.

Finally, there are two further sets of expectations in accordance with Lemmas 2 and 4: on the one hand, the buyer might expect always the high-value/high-cost outcome to be implemented, i.e., $\Lambda\left(C_{E, \mathcal{A}}(\bar{p})\right)=\left\{\left(v_{H}, \hat{p}_{0}^{H H H}\right),\left(v_{H}, \hat{p}_{L}^{H H H}\right),\left(v_{H}, \bar{p}\right)\right\}=: \Lambda^{H H H}$; on the other hand, the buyer might expect the worthless default to be renegotiated to the materially efficient outcome and the materially efficient outcome to be renegotiated to he high-value/high-cost outcome, i.e., $\Lambda\left(C_{E, \mathcal{A}}(\bar{p})\right)=\left\{\left(v_{L}, \hat{p}_{0}^{L H H}\right),\left(v_{H}, \hat{p}_{L}^{L H H}\right),\left(v_{H}, \bar{p}\right)\right\}=: \Lambda^{L H H}$. These expectations, however, are never part of the buyer's PPE. As we show in the Appendix A, expectations $\Lambda^{L H H}$ and $\Lambda^{H H H}$, which involve a high degree of price variation and relatively high mark-up prices for both the worthless and the materially efficient outcome, are less favorable from the buyer's perspective than expectations $\Lambda^{L L L}$ or $\Lambda^{L L H}$, which provide stability of value in combination with either moderate mark-ups or relatively few variations in prices.

Proposition 3. Consider $C=C_{E, \mathcal{A}}(\bar{p})$ with $|\mathcal{A}|<(n+1) / 2$. The SP-PPE consists of $\Lambda^{*}(C)=$ $\Lambda^{L L L}$ for $\lambda \leq \tilde{\lambda}_{E, \mathcal{A}}$ and $\Lambda^{*}(C)=\Lambda^{L L H}$ for $\lambda>\tilde{\lambda}_{E, \mathcal{A}}$.

Proposition 3 has some interesting implications regarding the likelihood of ex post inefficiency to occur, which the following corollary summarizes for the prominent case of a sales 
contract.

Corollary 2. Under a sales contract, $|\mathcal{A}|=1$, inefficient renegotiations become more likely (in the sense of set inclusion) as (i) $\lambda$ increases, or (ii) $n$ increases.

According to part (i), with a more loss-averse buyer it is less likely that the parties always agree upon implementing the materially efficient outcome ex post. The reason is that for a more loss-averse buyer it is more likely that not renegotiating in case of a high-value default outcome is a consistent behavior. Once the buyer gets attached to the idea of obtaining a high-value service at least in some states, it becomes too costly for the seller to compensate him in order to implement the materially efficient outcome. Regarding part (ii), note that for a sales contract we have $Q_{H}(E, \mathcal{A})=(n-1) / 2 n$. Thus, the environment becomes more certain as $n$ increases in the sense that it becomes less likely that the materially efficient outcome is the default. Since $\tilde{\lambda}_{E, \mathcal{A}}$ is decreasing in $n$, materially efficient trade is more likely to occur if the environment is rather uncertain, i.e., if $n$ is small. Intuitively, as is shown by Kőszegi and Rabin (2007), a loss-averse decision maker becomes less risk averse if the background risk increases, which is why here materially efficient trade is more likely to occur in equilibrium if $n$ is small.

\subsection{Employment Contracts with Large Acceptance Sets}

Suppose the buyer accepted an employment contract with a large acceptance set, $|\mathcal{A}|>(n+$ $1) / 2$, which encompasses the case of an employment relationship with maximum discretion $(\mathcal{A}=\mathcal{X})$. With every possible outcome $(v, c) \in\left\{(0,0),\left(v_{L}, c_{L}\right),\left(v_{H}, c_{H}\right)\right\}$ being a feasible choice in the acceptance set, there is no uncertainty about the default outcome under either type of employment contract.

Buyer employment contract.-For $E=B$, we have $\left(v^{D}\left(\theta, C_{B, \mathcal{A}}(\bar{p})\right), c^{D}\left(\theta, C_{B, \mathcal{A}}(\bar{p})\right)\right)=$ $\left(v_{H}, c_{H}\right)$ for all $\theta \in \Theta$, i.e., whenever renegotiations do not take place, the buyer chooses a materially inefficient high-value/high-cost service. The buyer's expectations regarding the outcome of renegotiation comprise a single value-price pair, $\Lambda\left(C_{B, \mathcal{A}}(\bar{p})\right)=\left\{\left(\hat{v}_{H}, \hat{p}_{H}\right)\right\}$.

First, suppose the buyer expects materially efficient renegotiation to occur and denote these expectations by

$$
\Lambda\left(C_{B, \mathcal{A}}(\bar{p})\right)=\left\{\left(v_{L}, \hat{p}_{H}^{L}\right)\right\}=: \Lambda_{B}^{L}
$$

Since renegotiation involves a decrease in value, the buyer has to expect to be offered a discount price $\hat{p}_{H}^{L}<\bar{p}$. The only price compatible with equilibrium-equating the buyer's utility from accepting the seller's renegotiation offer, $U\left(v_{L}, \hat{p}_{H}^{L} \mid \Lambda_{B}^{L}\right)=v_{L}-\hat{p}_{H}^{L}$, and his utility from rejecting renegotiation, $U\left(v_{H}, \bar{p} \mid \Lambda_{B}^{L}\right)=v_{H}-\bar{p}-\lambda\left(\bar{p}-\hat{p}_{H}^{L}\right)$-is

$$
\hat{p}_{H}^{L}=\bar{p}-\frac{v_{H}-v_{L}}{1+\lambda} .
$$

With the associated profits for the seller amounting to $\hat{p}_{H}^{L}-c_{L}$, there is no profitable deviation: first, not offering renegotiation at all results in strictly lower profits $\bar{p}-c_{H}$; second, according to 
Corollary 1 , offering renegotiations to a worthless service is not profitable for the seller. Thus, as for employment contracts with small acceptance sets, there always is a SP-PE with materially efficient renegotiation. The buyer's expected utility in this equilibrium amounts to

$$
E U\left(\Lambda_{B}^{L}\right)=v_{L}-\bar{p}+\frac{v_{H}-v_{L}}{1+\lambda} .
$$

On the other hand, the buyer might expect renegotiation not to occur and thus to obtain a high-value service at price $\bar{p}$, i.e.,

$$
\Lambda\left(C_{B, \mathcal{A}}(\bar{p})\right)=\left\{\left(v_{H}, \bar{p}\right)\right\}=: \Lambda_{B}^{H}
$$

Despite these expectations, at the renegotiation stage the seller might offer to deliver the efficient service at a discount price. The buyer accepts this offer for any price below $p^{R}=\bar{p}-(1+$ $\lambda)\left(v_{H}-v_{L}\right)$, at which he is indifferent between accepting and rejecting the seller's renegotiation offer, i.e., $U\left(v_{L}, p^{R} \mid \Lambda_{B}^{H}\right)=v_{L}-p^{R}-\lambda\left(v_{H}-v_{L}\right)$ equals $U\left(v_{H}, \bar{p} \mid \Lambda_{B}^{H}\right)=v_{H}-\bar{p}$. The seller has no incentive to deviate from the buyer's expectation if $p^{R}-c_{L} \leq \bar{p}-c_{H}$ or, equivalently, if the buyer's loss aversion is rather strong, i.e., $\lambda \geq \tilde{\lambda}_{E, \mathcal{A}}$. Since renegotiation to the delivery of a worthless service never is profitable for the seller (cf. Corrolary 1), $\lambda \geq \tilde{\lambda}_{E, \mathcal{A}}$ not only is necessary but also sufficient for existence of a SP-PE in which renegotiations never take place. The buyer's expected utility in this equilibrium is

$$
E U\left(\Lambda_{B}^{H}\right)=v_{H}-\bar{p}
$$

Finally, by Lemma 2, the buyer expecting renegotiation to result in the delivery of a worthless service, $\hat{v}_{H}=0$, is incompatible with equilibrium.

Comparison of (25) and (27) reveals that for $\lambda>0$ the buyer strictly prefers the materially inefficient no-renegotiation outcome. Expecting no renegotiation to occur is consistent with equilibrium, however, only if the buyer's attachment to high value, captured by his degree of loss aversion $\lambda$, is sufficiently strong.

Proposition 4. Consider $C=C_{B, \mathcal{A}}(\bar{p})$ with $|\mathcal{A}|>(n+1) / 2$.

(i) There always exists a $S P-P E$ with $\Lambda^{*}(C)=\Lambda_{B}^{L}$.

(ii) The SP-PPE consists of $\Lambda^{*}(C)=\Lambda_{B}^{L}$ if $\lambda<\tilde{\lambda}_{B, \mathcal{A}}$ and $\Lambda^{*}(C)=\Lambda_{B}^{H}$ if $\lambda \geq \tilde{\lambda}_{B, \mathcal{A}}$.

According to Proposition 4, while always constituting a SP-PE, materially efficient renegotiations correspond to the SP-PPE only for mild degrees of loss aversion. The reason is that if the buyer is loss averse and expects materially efficient renegotiations to occur, the necessary reduction in price to make him willing to accept materially efficient renegotiations is lower than the reduction in value $v_{H}-v_{L}$. This is the case because the buyer suffers more from not obtaining the discount price than he gains from obtaining the high-value service. In consequence, the buyer is better off by not expecting to renegotiate the contract with the seller in the first place. This is a credible plan, however, only if the buyer is sufficiently loss averse. 
Note that a buyer employment contract with large acceptance set achieves the maximum feasible overall surplus if $\lambda<1-\left(c_{H}-c_{L}\right) /\left(v_{H}-v_{L}\right)=\tilde{\lambda}_{B, \mathcal{A}}$ : on the one hand, there is always materially efficient trade ex post, and, on the other hand, any losses are eliminated because the buyer knows for sure that he will pay $\hat{p}_{H}^{L}$ and will obtain value $v_{L}$. Thus, if the buyer is only mildly loss averse, a buyer employment contract with large acceptance set represents an optimal contract. Finally, note that (in contrast to small acceptance sets) the likelihood of materially efficient renegotiations to occur is independent of the complexity of the environment $n$.

Seller employment contract.- For $E=S$, we have $\left(v^{D}\left(\theta, C_{S, \mathcal{A}}(\bar{p})\right), c^{D}\left(\theta, C_{S, \mathcal{A}}(\bar{p})\right)\right)=$ $(0,0)$ for all $\theta \in \Theta$, i.e., the seller always opts for the provision of a no cost (worthless) service. Again, the buyer's expectations regarding the outcome of renegotiation comprise a single valueprice pair, $\Lambda\left(C_{S, \mathcal{A}}(\bar{p})\right)=\left\{\hat{v}_{0}, \hat{p}_{0}\right\}$.

Suppose the buyer expects materially efficient renegotiations to occur,

$$
\Lambda^{L}\left(C_{S, \mathcal{A}}(\bar{p})\right)=\left\{\left(v_{L}, \hat{p}_{0}^{L}\right)\right\}=: \Lambda_{S}^{L} .
$$

Since renegotiation involve an increase in the seller's cost, the buyer has to expect to be demanded a mark-up $\hat{p}_{0}^{L}-\bar{p}>0$. The highest price the seller can charge so that the buyer accepts her offer-given that this price is also the buyer's reference point-is

$$
\hat{p}_{0}^{L}=\bar{p}+(1+\lambda) v_{L} .
$$

With the seller's profits amounting to $\hat{p}_{0}^{L}-c_{L}$, there is no profitable deviation: first, not offering renegotiation at all results in strictly lower profits equal to $\bar{p}$; second, the highest price at which the buyer would be willing to accept a high-value/high-cost service equals $p^{R}=\hat{p}_{0}^{L}+\left(v_{H}-\right.$ $\left.v_{L}\right) /(1+\lambda)$, which results in strictly lower profits $p^{R}-c_{H}$ than offering to deliver the efficient service at price $\hat{p}_{0}^{L}$. Thus, materially efficient renegotiation always constitutes a SP-PE that results in expected utility

$$
E U\left(\Lambda_{S}^{L}\right)=-\lambda v_{L}-\bar{p}
$$

Next, note that the buyer expecting renegotiation not to be offered-i.e., to obtain zero value at price $\bar{p}$, cannot be an equilibrium by Lemma 2 .

Finally, the buyer might expect the seller to offer renegotiation to the materially inefficient high-value/high-cost service,

$$
\Lambda^{H}\left(C_{S, \mathcal{A}}(\bar{p})\right)=\left\{\left(v_{H}, \hat{p}_{0}^{H}\right)\right\}=: \Lambda_{S}^{H} .
$$

The only price $\hat{p}_{0}^{H}>\bar{p}$ compatible with such an equilibrium is

$$
\hat{p}_{0}^{H}=\bar{p}+(1+\lambda) v_{H}
$$

Whenever these expectations are consistent with sequential rationality, which is the case for $\lambda$ sufficiently large, there exists a SP-PE of this type. The buyer's expected utility in this equilibrium is

$$
E U\left(\Lambda_{S}^{H}\right)=-\lambda v_{H}-\bar{p}
$$


Regarding the buyer's PPE, comparison of (30) and (33) reveals that the buyer strictly prefers materially efficient renegotiation.

Proposition 5. Consider $C=C_{S, \mathcal{A}}(\bar{p})$ with $|\mathcal{A}|>(n+1) / 2$. There always exists a SP-PE with $\Lambda^{*}(C)=\Lambda_{S}^{L}$. Moreover, this is also the SP-PPE for all $\lambda \geq 0$.

In our setup, a seller employment contract with a large acceptance set always is an optimal contract. It always leads to the implementation of the materially efficient service ex post. Furthermore, there is no uncertainty for the buyer, neither regarding the ultimate price he has to pay, nor regarding his valuation of the delivered service. Thus, the buyer does not incur a loss on the equilibrium path.

The finding that under a seller employment contract with complete acceptance set materially efficient renegotiation always takes place at first glance seems in contrast to Herweg and Schmidt (2013). The strong finding in favor of a seller employment contract, however, is due to Assumption 1. If Assumption 1 does not hold, no renegotiation-i.e., trading the worthless service in each state at price $\bar{p}$, may constitute a SP-PE. Suppose the buyer expects renegotiation not to occur, i.e.,

$$
\Lambda\left(C_{S, \mathcal{A}}(\bar{p})\right)=\{(0, \bar{p})\}=: \Lambda_{S}^{0}
$$

The buyer's expected utility in this case is

$$
E U\left(\Lambda_{S}^{0}\right)=-\bar{p}
$$

which is higher than $E U\left(\Lambda_{S}^{L}\right)$ and $E U\left(\Lambda_{S}^{H}\right)$. Thus, whenever $\Lambda_{S}^{0}$ constitutes a credible plan, it constitutes the SP-PPE. For $\Lambda_{S}^{0}$ to be a credible plan, there must not be the possibility for the seller to offer renegotiation to a service with strictly positive value $v \in\left\{v_{L}, v_{H}\right\}$. The buyer rejects any such offer at price $p^{R}$ if

$$
v-p^{R}-\lambda\left(p^{R}-\bar{p}\right) \leq-\bar{p}
$$

If the above inequality holds for the materially efficient service under the lowest possible renegotiation price the seller finds profitable to offer in this case, i.e., $v=v_{L}$ and $p^{R}=\bar{p}+c_{L}$, then it holds for the high-value/ high-cost service, accordingly. Thus, no renegotiation is a credible plan if and only if $v_{L} /(1+\lambda) \leq c_{L}$.

Observation 2. Suppose $v_{L} \leq 2 c_{L}$. Consider $C=C_{S, \mathcal{A}}(\bar{p})$ with $|\mathcal{A}|>(n+1) / 2$.

(i) There always exists a SP-PE with $\Lambda^{*}(C)=\Lambda_{S}^{L}$.

(ii) The SP-PPE consists of $\Lambda^{*}(C)=\Lambda_{S}^{L}$ if $\lambda<\left(v_{L}-c_{L}\right) / c_{L}$ and $\Lambda^{*}(C)=\Lambda_{S}^{0}$ if $\lambda \geq$ $\left(v_{L}-c_{L}\right) / c_{L}$.

If Assumption 1 is relaxed, a similar structure can be found for the seller employment contract with a large acceptance set as for the corresponding buyer employment contract. The loss-averse buyer is exploited by the seller when renegotiation occurs. Thus, the buyer is better off by not expecting to renegotiate the contract with the seller, which is a credible plan if the buyer is sufficiently loss averse. 


\subsection{Employment Contracts with Medium Acceptance Sets}

Buyer employment contract.-For $E=B$ at least one service in the acceptance set yields a value of at least $v_{L}$. With the buyer always opting for the maximum value in case that renegotiation does not occur, the default outcome is $\left(v_{L}, c_{L}\right)$ in some states and $\left(v_{H}, c_{H}\right)$ in others.

With Lemmas 2 and 4 ruling out that a worthless outcome prevails as well as criss-cross renegotiations, we are left with three sets of expectations as potential equilibrium candidates. First, the buyer might expect renegotiation always to result in delivery of the materially efficient service, i.e., $\Lambda\left(C_{B, \mathcal{A}}(\bar{p})\right)=\left\{\left(v_{L}, \bar{p}\right),\left(v_{L}, \hat{p}_{H}^{L L}\right)\right\}=: \Lambda_{B}^{L L}$. As with a large acceptance set, materially efficient renegotiation always constitutes a SP-PE. The second set of expectations potentially consistent with equilibrium is that renegotiation never occur, i.e., $\Lambda\left(C_{B, \mathcal{A}}(\bar{p})\right)=$ $\left\{\left(v_{L}, \bar{p}\right),\left(v_{H}, \bar{p}\right)\right\}=: \Lambda_{B}^{L H}$. Finally, the buyer might expect renegotiation always to result in the delivery of a materially inefficient high-value/high-cost service, i.e., $\Lambda\left(C_{B, \mathcal{A}}(\bar{p})\right)=$ $\left\{\left(v_{H}, \hat{p}_{L}^{H H}\right),\left(v_{H}, \bar{p}\right)\right\}=: \Lambda_{B}^{H H}$.

Comparison of the buyer's expected utility across these sets of expectations reveals that the buyer prefers renegotiation not to occur $\left(\Lambda_{B}^{L H}\right)$ whenever expecting renegotiation not to occur constitutes a SP-PE. The buyer expecting renegotiation not to occur is consistent with SP-PE only for a rather high degree of loss aversion, i.e., for $\lambda \geq \tilde{\lambda}_{B, \mathcal{A}}$. Thus, a strongly loss-averse buyer prefers certainty in the price dimension over certainty in the value dimension. ${ }^{18}$ For $\lambda<\tilde{\lambda}_{B, \mathcal{A}}$, on the other hand, materially efficient renegotiations $\left(\Lambda_{B}^{L L}\right)$ support the SP-PPE. Thus, while inefficient renegotiations $\left(\Lambda_{B}^{H H}\right)$ may be consistent with SP-PE for a mildly lossaverse buyer, he prefers low-value stability with occasional price concessions over high-value stability with occasional price mark-ups.

Seller employment contract.-With the seller always opting for the minimum cost in case that renegotiation does not occur, the default outcome in some states is $(0,0)$ and in other states it is $\left(v_{L}, c_{L}\right)$.

Suppose the buyer expects renegotiation to result in the delivery of the materially efficient service, i.e., $\Lambda\left(C_{S, \mathcal{A}}(\bar{p})\right)=\left\{\left(v_{L}, \hat{p}_{0}^{L L}\right),\left(v_{L}, \bar{p}\right)\right\}=: \Lambda_{S}^{L L}$. The buyer expecting materially efficient renegotiation leaves no room for any profitable deviation of the seller, i.e., $\Lambda_{S}^{L L}$ is a SP-PE. In accordance with Lemmas 2 and 4, the buyer might hold two further sets of expectations: $\Lambda\left(C_{S, \mathcal{A}}(\bar{p})\right)=\left\{\left(v_{H}, \hat{p}_{0}^{H H}\right),\left(v_{H}, \hat{p}_{L}^{H H}\right)\right\}=: \Lambda_{S}^{H H}$ or $\Lambda\left(C_{S, \mathcal{A}}(\bar{p})\right)=\left\{\left(v_{L}, \hat{p}_{0}^{L H}\right),\left(v_{H}, \hat{p}_{L}^{L H}\right)\right\}=:$ $\Lambda_{S}^{L H}$. While these expectations are consistent with sequential rationality if the buyer is sufficiently loss averse, his expected utility under these expectations is strictly lower than under materially efficient renegotiation because of the high mark-up prices associated with renegotiations to the high-value/high-cost outcome.

Proposition 6. Consider $C=C_{E, \mathcal{A}}(\bar{p})$ with $|\mathcal{A}|=(n+1) / 2$.

\footnotetext{
${ }^{18} \mathrm{~A}$ somewhat similar finding - that an expectation-based loss-averse decision maker with a universal gain-loss function dislikes fluctuations in prices more than he dislikes fluctuations in values-is obtained by Heidhues and Köszegi (2008), and in fact it is a major driver of their "focal pricing" result.
} 
(i) For $E=B$ the SP-PPE consists of $\Lambda^{*}(C)=\Lambda_{B}^{L L}$ if $\lambda \leq \tilde{\lambda}_{B, \mathcal{A}}$ and $\Lambda^{*}(C)=\Lambda_{B}^{L H}$ if $\lambda>\tilde{\lambda}_{B, \mathcal{A}}$. Moreover, there always exists a $S P-P E$ with $\Lambda^{*}(C)=\Lambda_{B}^{L L}$.

(ii) For $E=S$ the SP-PPE consists of $\Lambda^{*}(C)=\Lambda_{S}^{L L}$ for all $\lambda$.

With a medium acceptance set a seller employment contract ensures that the materially efficient good is always traded ex post. If the buyer is only mildly loss averse, materially efficient trade takes also always place under a buyer employment contract with medium acceptance set. These contracts are nevertheless not optimal because they lead to variations in prices caused by the uncertain default outcome. Due to the uncertainty in the price dimension the buyer ex ante expects to incur losses, which reduces the ex ante expected surplus from contracting below the maximal achievable surplus.

\subsection{The Optimal Long-Term Contract}

At date 0 , both sellers submit a long-term contract offer to the buyer. With sellers competing for the buyer's favor, spot contracting, which is rather unfavorable from the buyer's perspective, will not occur in equilibrium. The Bertrand nature of seller competition at date 0 makes sellers offer contracts that maximize the buyer's expected utility subject to the constraint that expected profits do not fall short of the fleeting outside option. The most attractive long-term contract a seller can offer therefore pursues ex post material efficiency and minimizes the buyer's expected losses associated with renegotiation. This can be achieved only by employment contracts with a sufficiently large acceptance set. For $|\mathcal{A}|>(n+1) / 2$, the trade price which equates a seller's profits with the profits from the outside option is

$$
\bar{p}(B, \mathcal{A})=\pi+c_{L}+\frac{v_{H}-v_{L}}{1+\lambda}
$$

for the buyer employment contract and

$$
\bar{p}(S, \mathcal{A})=\pi+c_{L}-(1+\lambda) v_{L}
$$

for the seller employment contract.

Proposition 7. In a SP-PPE, seller $i=1,2$ offers a contract $C=C_{E_{i}, \mathcal{A}_{i}}\left(\bar{p}\left(E_{i}, \mathcal{A}_{i}\right)\right)$ with $|\mathcal{A}|>(n+1) / 2$ and (i) $E_{i} \in\{S, B\}$ if $\lambda<\tilde{\lambda}_{B, \mathcal{A}}$, (ii) $E_{i}=S$ if $\lambda \geq \tilde{\lambda}_{B, \mathcal{A}}$.

Under the standard approach of the incomplete contracting literature, i.e., with a loss-neutral buyer, each form of long-term contract is optimal irrespective of the size of the acceptance set or which party is designated to choose (cf. Observation 1). In particular, also a sales contract is optimal. According to Proposition 7, with a loss-averse buyer, in contrast, only employment contracts with sufficiently large acceptance sets prevail in equilibrium-e.g., employment relationships with a maximum degree of discretion in form of a complete acceptance set. Thus, our theory provides a rationale for the employment relationship if renegotiations are feasible even in the absence of non-contractible investments. Keep in mind that the strict optimality of 
a seller-employment contract with a large acceptance set is due to Assumption 1. If we relax Assumption 1, the seller employment contract is not necessarily optimal for high degrees of loss aversion. In a more general model, for high degrees of buyer loss aversion, we cannot expect that the parties can achieve both: material efficiency and no losses.

How can the optimality of a seller employment contract with large acceptance set be interpreted in terms of a practical example? Suppose the buyer owns a car which needs to be repaired and the seller operates a car repair shop. Ex ante, it is uncertain to what extent each feasible treatment increases the value of the car and how costly it is. The seller employment contract can be interpreted as a situation where the customer writes a contract with the car repair shop, so that the car repair shop performs only the treatments necessary that the car is roadworthy again at some prespecified price $\bar{p}$. Let the gains from trade if the car is fixed so that it is just roadworthy be normalized to zero. Before the customer picks his car up, however, the shop calls and tells him that it would be reasonable-even though not necessary in a strict sense- to do this and that additional repair, which increases the value of the car but would also be somewhat more costly. The car repair shop proposes the materially efficient service at price $\hat{p}>\bar{p}$ which is accepted by the customer. In this example, as in many casual observations, the ultimate bill of the car repair shop is higher than the initially specified price. This happens even though the customer is loss averse and therefore suffers from unexpected price increases. The reason for this nevertheless to happen is that the customer rationally anticipates that the initial contract will be renegotiated and that he will ultimately pay a higher price than originally specified.

The comparison of employment contracts and sales contracts goes back to Simon (1951). While both forms of contractual arrangement fix a trade price, the former leaves a lot of discretion to the "employer" to tell the "employee" ex post which specification of the service will be traded, whereas the latter also specifies the nature of the service ex ante. According to Simon, the advantage of the first is its flexibility, which allows for the delivered service to be efficiently adjusted to the state of the world. On the other hand, this flexibility, which is absent under a sales contract, at the same time makes the employment contract prone to abuse by the employer, who will be tempted to choose his/her most preferred service instead of the efficient service. This trade-off in the choice of the optimal long-term contract, however, disappears if Coasian bargaining is feasible ex post-i.e., if the parties can renegotiate the initial contract (Bolton and Dewatripont, 2005). In our setup, the advantage of an employment contract is indeed rooted in its flexibility. The channel through which this flexibility benefits the trading relationship goes beyond ensuring materially efficient trade-remember that if the buyer is only mildly loss averse, the materially efficient outcome is implemented under a sales contract as well. The advantage of the employment contract rather is that it makes the default outcome under renegotiations deterministic and thereby achieves to eliminate any losses in value or prices otherwise incurred by the buyer. Nevertheless, if one picks the "wrong" employment contract, then also abuse as discussed by Simon appears in our setting. Under a buyer employment contract, if the 
buyer is highly loss averse, he picks an inefficient high-value/high-cost service and is unwilling to renegotiate away from this outcome. In this case, the seller always has to provide one of the very costly services and therefore is-in a sense-abused by the buyer.

\section{Non-Contractible Investments And The Hold-up Problem}

In this section, we augment our model by allowing the buyer to make a non-contractible investment at date 1 that increases his valuation at date 5 in the spirit of Hart and Moore (1988). Investment $I \in\{0,1\}$ is associated with cost $\psi I$ for the buyer, where $\psi>0$. The investment increases the buyer's valuation from any valuable service but not from the provision of a worthless service. Moreover, the investment is particularly effective if the materially efficient service is traded ex post. Formally, the buyer's valuation now depends on his investment, $v_{k}(I)$ with $k \in\{0, L, H\}$, and we have

$$
v_{L}(1)-v_{L}(0)>v_{H}(1)-v_{H}(0)>0=v_{0}(1)=v_{0}(0) .
$$

Furthermore, next to Assumption 1 being satisfied for all $I$, we impose the following assumption, which ensures that a loss-neutral buyer's investment incentives are higher under a buyer employment contract with large acceptance set than under a sales contract.

\section{Assumption 3.}

$$
\frac{v_{L}(1)-v_{L}(0)}{v_{H}(1)-v_{H}(0)}<\frac{n+1}{2} .
$$

The sellers' costs are independent of the buyer's investment decision. In addition, even though non-contractible, the buyer's investment choice is observed by all sellers at the beginning of date 2 .

Loss-Neutral Buyer and First-Best Investments.-The joint surplus is maximized for $I=1$ if investing leads to an increase in joint surplus from materially efficient trade that exceeds the buyer's investment cost, i.e., if

$$
\psi \leq v_{L}(1)-v_{L}(0)=: \psi^{F B}
$$

Due to the frictions caused by incomplete contracting, however, the buyer's investment incentives are inefficiently too low. In the following, we investigate which type of incomplete contract maximizes the buyer's investment incentives. ${ }^{19}$

Note that with ex post trade being always efficient for $\lambda=0$, the optimal long-term contract maximizes the investment incentives for the buyer. Moreover, any type of seller employment contract leads to lower investment incentives than the corresponding buyer employment contract with the same size of the acceptance set. In particular, under a seller employment contract with

\footnotetext{
${ }^{19}$ We say that a contract maximizes the buyer's investment incentives if it maximizes the range of investment costs $\psi$ for which the buyer chooses $I=1$.
} 
large acceptance set (just as under a spot contract) the buyer has no incentives to invest because the seller has all the bargaining power ex post.

Under a buyer employment contract with large acceptance set the buyer invests if $\psi \leq \psi_{B, \mathcal{L}}$, where

$$
\psi_{B, \mathcal{L}}:=v_{H}(1)-v_{H}(0) .
$$

Under a buyer employment contract with small (or medium) acceptance set, the buyer invests if $\psi \leq \psi_{B, \mathcal{S}}(s)$, where

$$
\psi_{B, \mathcal{S}}(s):=Q_{L}(B, \mathcal{A})\left[v_{L}(1)-v_{L}(0)\right]+Q_{H}(B, \mathcal{A})\left[v_{H}(1)-v_{H}(0)\right]
$$

and $s=\frac{n+1}{2}-|\mathcal{A}| \in\{0, \ldots,(n-1) / 2\}$ is inversely related to the size of the acceptance set according to (12) and (13). Note that $s=0$ corresponds to a buyer employment contract with medium acceptance set. With $Q_{H}$ being decreasing and $Q_{L}$ being independent of $s$ it follows immediately that $s=0$ maximizes the investment incentives. In addition, $\psi_{B, \mathcal{S}}(0)>\psi_{B, \mathcal{L}}>$ $\psi_{B, \mathcal{S}}\left(\frac{n-1}{2}\right)$.

Observation 3. Suppose the buyer is not loss averse. Then, the range of investment costs for which the buyer undertakes the investment is maximized under a buyer employment contract with medium acceptance set.

\subsection{Loss Aversion and the Hold-Up Problem}

Next, we investigate how buyer loss aversion affects the hold-up problem and which contract creates the highest incentives to invest. While we do not solve for the overall optimal long-term contract explicitly, we discuss the trade-offs between ex post material efficiency, induced losses, and ex ante investments that the choice of the optimal long-term contract typically involves.

Any type of seller employment contract is dominated by a buyer employment contract with large acceptance set. ${ }^{20}$ Moreover, without investment, if the buyer's degree of loss aversion is moderate, a buyer employment contract with large acceptance set eliminates any losses and thus is an optimal contract. Therefore, we first analyze the investment incentives created by this type of contract in detail. Thereafter, we address buyer employment contracts with small and medium acceptance sets.

Buyer employment contract with large acceptance set.-When deciding at date 1 whether to invest or not, the buyer takes into account how his investment decision affects his reference point such that he never feels a loss in the "investment cost" dimension. For a given level of investment, the SP-PPE is materially efficient renegotiations if

$$
\lambda<\frac{c_{H}-c_{L}}{v_{H}(I)-v_{L}(I)}-1=: \tilde{\lambda}_{B, \mathcal{L}}(I)
$$

\footnotetext{
${ }^{20} \mathrm{~A}$ buyer employment contract with large acceptance set not only leads to higher investment incentives (as in the case of a loss-neutral buyer) but also induces (weakly) lower losses than any type of seller employment contract.
} 
and no renegotiation otherwise. The $\lambda$-threshold depends on the buyer's investment decision and, therefore, is now endogenous. By the assumption implied by (39), we have $\tilde{\lambda}_{B, \mathcal{L}}(1)>$ $\tilde{\lambda}_{B, \mathcal{L}}(0)$, i.e., investment by the buyer reduces the range for which no renegotiation to take place constitutes a SP-PE. Hence, three cases have to be considered: (i) For mild degrees of loss aversion, efficient renegotiation takes always place independent of the buyer's investment decision. (ii) For intermediate degrees of loss aversion, materially efficient renegotiation takes place ex post only if the buyer undertakes the investment ex ante. (iii) For high degrees of loss aversion renegotiation does not take place independent of the investment choice.

First, for $\lambda<\tilde{\lambda}_{B, \mathcal{L}}(0)$, by (25) the buyer undertakes the investment if

$$
\psi \leq \psi_{B, \mathcal{L}}+\frac{\lambda}{1+\lambda}\left\{\left[v_{L}(1)-v_{L}(0)\right]-\left[v_{H}(1)-v_{H}(0)\right]\right\}=: \psi_{B, \mathcal{L}}(\lambda) .
$$

Note that buyer loss aversion here enhances the investment incentives and thus reduces the hold-up problem, i.e., $\partial \psi_{B, \mathcal{L}} / \partial \lambda>0$. At first glance, this might be surprising because the buyer is exploited by the seller in the SP-PE with efficient renegotiations. In order to understand this, note that by (39), the price reduction the seller offers to the buyer in order to implement the materially efficient service is decreasing in the investment-cf.(24). This reduction in the discount, however, is less pronounced for a loss-averse buyer who already receives a smaller discount without investment. This explains why investment incentives are increasing in the degree of loss aversion. Yet, the first-best investment level is never reached since the seller has all the bargaining power ex post.

Second, in the intermediate case, $\tilde{\lambda}_{B, \mathcal{L}}(0) \leq \lambda<\tilde{\lambda}_{B, \mathcal{L}}(1)$, renegotiation takes place if the buyer undertakes the investment but otherwise a high-value service is always traded. By (25) and (27) the buyer prefers to invest if

$$
\psi \leq \psi_{B, \mathcal{L}}-\frac{\lambda}{1+\lambda}\left[v_{H}(1)-v_{L}(1)\right] .
$$

Note that in this intermediate case investment incentives are lowest and the buyer's willingness to invest is decreasing in his degree of loss aversion.

Finally, for $\lambda \geq \tilde{\lambda}_{B, \mathcal{L}}(1)$ a high-value services is always traded irrespective of whether the buyer undertakes the investment. Hence, by (27), the buyer invests if $\psi \leq \psi_{B, \mathcal{L}}$.

The buyer's optimal investment decision as function of his degree of loss aversion depending on the investment costs is depicted in Figure 3. The intuition behind the downward discontinuity at $\tilde{\lambda}_{B, \mathcal{L}}(0)$ is as follows: The buyer always prefers no renegotiations to occur over materially efficient renegotiations because in the latter case the seller exploits the buyer's loss aversion ex post. Expecting no renegotiations to take place, however, is not always a credible plan-in particular, if the buyer invests, he is always willing to renegotiate to the materially efficient service because the investment increases the buyer's value from materially efficient trade more than it increases his value from a high-value service. In other words, not undertaking the investment is a commitment for the buyer not to renegotiate the contract ex post, which protects him from ex post opportunism by the seller. 


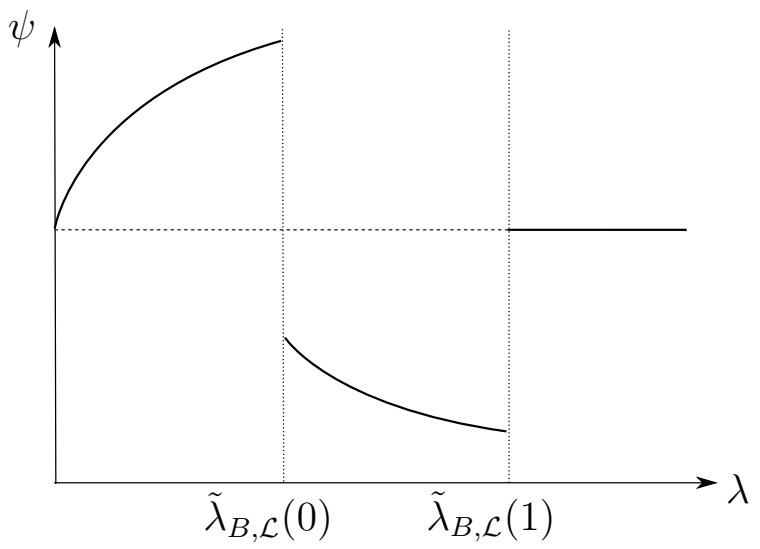

Figure 3: Critical Investment cost for a large acceptance set

Maximum investment incentives. - We now investigate the optimal size of the acceptance set of the buyer employment contract in order to achieve the highest investment incentives. We restrict attention to $\lambda<\tilde{\lambda}_{B, \mathcal{L}}(0)$, so that materially efficient renegotiation always takes place and thus the case of a loss-neutral buyer represents a clear benchmark.

Suppose the buyer signed a buyer employment contract with a small acceptance set. From (19) it is readily established that in this case the buyer undertakes the investment only if $\psi \leq$ $\psi_{B, \mathcal{S}}(s, \lambda)$, where

$$
\begin{aligned}
\psi_{B, \mathcal{S}}(s, \lambda) & :=\left[v_{L}(1)-v_{L}(0)\right]\left[1-Q_{0}(B, \mathcal{A})(1+\lambda)\right] \\
& -Q_{H}(B, \mathcal{A}) \frac{1-\lambda\left[1-Q_{H}(B, \mathcal{A})\right]}{1+\lambda Q_{H}(B, \mathcal{A})}\left\{\left[v_{L}(1)-v_{L}(0)\right]-\left[v_{H}(1)-v_{H}(0)\right]\right\} .
\end{aligned}
$$

With $Q_{H}=(n-1-s) / n, Q_{L}=1 / n$, and $Q_{0}=s / n$ for $s \in\{0, \ldots,(n-1) / 2\}, s=0$ corresponds to a medium acceptance set and $s=(n-1) / 2$ to a sales contract. As we show in the Appendix A, $\psi_{B, \mathcal{S}}(s, \cdot)$ is maximized at $s=0$. Decreasing $s$-and thereby increasing the acceptance set-reduces the probability of the worthless outcome being the default and therefore enhances the buyer's willingness to invest. Increasing the acceptance set beyond a medium acceptance set, however, reduces investment incentives, i.e., $\left.\psi_{B, \mathcal{S}}(s, \lambda)\right|_{s=0}>\psi_{B, \mathcal{L}}(\lambda)$. This is because the materially efficient outcome as default leads to higher investment incentives than the high-value outcome as default. Thus, the buyer's willingness to invest is maximized for a buyer employment contract with a medium acceptance set.

Whether loss aversion increases or decreases the buyer's investment incentives crucially depends on the size of the acceptance set. For the extreme cases of buyer employment contract with a medium acceptance set and a sales contract, tedious but straightforward calculations show that

$$
\left.\frac{\partial \psi_{B, \mathcal{S}}}{\partial \lambda}\right|_{s=0}>0 \quad \text { and }\left.\quad \frac{\partial \psi_{B, \mathcal{S}}}{\partial \lambda}\right|_{s=\frac{n-1}{2}}<0 .
$$

As suggested by this observation, buyer loss aversion indeed reduces the hold-up problem by enhancing investment incentives only if the buyer employment contract specifies a sufficiently large acceptance set. 
Proposition 8. Suppose $\lambda<\tilde{\lambda}_{B, \mathcal{L}}(0)$. Then, the range of investment costs for which the buyer undertakes the investment is maximized under a buyer employment contract with medium acceptance set. Furthermore, there exists $K \in\left(0, \frac{n+1}{2}\right)$ such that the range of investment costs for which the buyer invests increases in his degree of loss aversion if and only if $|\mathcal{A}| \geq K$.

The finding that a buyer employment contract with medium acceptance set maximizes the buyer's willingness to invest does not imply that this is the optimal contract that is offered in the SP-PPE. The reason is that a buyer employment contract with medium acceptance set leads to expected losses for the buyer because the default is uncertain. Therefore, as long as a buyer employment contract with large acceptance set makes the buyer undertake the investment, this is the optimal contract because its unique default outcome eliminates any losses. For high investment costs, so that the buyer undertakes the investment only for a buyer employment contract with medium acceptance set but not for one with a large acceptance set, there is a tradeoff between enhancing investments and minimizing losses. Depending on how the efficiency gain of investments compares to the induced losses from an uncertain default outcome, both a buyer employment contract with medium as well as with a large acceptance set can be optimalin the sense of maximizing the overall surplus — and thus be part of the SP-PPE.

\section{CONClusion}

In this paper, we analyze a simple trading relationship between a loss-averse buyer and a profitmaximizing seller. The gains from trading a particular service depend on the state of nature, which is unknown to the parties at the point in time when writing a long-term contract. Following the literature on incomplete contracts, the parties are restricted to write contracts that do not condition on the state of nature. Moreover, whether trade has taken place and whose fault it was if not can be verified by a court.

We show that the buyer's expectations regarding the ex post implemented service are crucial for the outcome of renegotiation. In particular, independent of the type of the concluded contract, if the buyer expects that renegotiation takes place when needed to implement the materially efficient service, it is indeed an equilibrium that materially efficient renegotiation takes always place. Materially efficient renegotiation is, however, not always in the buyer's interest ex ante. When expecting that materially efficient renegotiation takes always place, the buyer is, for instance, willing to renegotiate away from a service which leads to a higher value at a relatively moderate price reduction. In consequence, the seller may be able to exploit the buyer's loss aversion at the renegotiation stage. Since this is anticipated by the buyer, if the buyer is severely loss averse, his preferred and also credible plan is not always to renegotiate to the materially efficient service. In these cases, loss aversion leads to material inefficiencies ex post. Ex ante, as we argue, the parties write a long-term contract that maximizes the expected utility of both parties, i.e., the optimal long-term contract achieves a high material efficiency without imposing severe expected losses on the buyer. In this regard, the optimal long-term contract is 
an employment contract with a large acceptance set. The advantage of such a contract is its unambiguous default outcome, which eliminates any variations in valuations and prices, thereby minimizing expected losses.

In an extension, we allow the buyer to make a relationship-specific investment. The investment made by the buyer increases the gains from trade-in particular, from materially efficient trade. With the investment cost being sunk at the renegotiation stage, the seller has an incentive to hold up the buyer. We characterize situations in which buyer loss aversion can reduce the hold-up problem and thereby enhances investment incentives.

Admittedly, regarding several aspects, our model is fairly stylized. Solving this model, however, requires an involved case-by-case analysis. Many of the simplifying assumptions are imposed in order to reduce the number of case distinctions and are not crucial for our main findings.

First, we presume that only the buyer is loss averse. Assuming that the seller is also expectationbased loss averse does not change the main insights. The interesting effects in our model arise because the seller, who has all the bargaining power at the renegotiation stage, may exploit the buyer's loss aversion. If the seller is also loss-averse, an employment contract with large acceptance set is still optimal, even if the buyer is allowed to freely choose the service ex post. When the seller expects materially efficient renegotiation, she incurs a loss when delivering a highcost service. The loss-averse buyer is willing to renegotiate away from a high-value service to the materially efficient service for a reduction in the price that is lower than the reduction in his valuation if he expected materially efficient trade. Thus, it is still an equilibrium that materially efficient trade takes always place. Moreover, this is also the preferred personal equilibrium as long as the buyer's degree of loss aversion is not too severe. In addition, under a seller employment contract, in the SP-PPE materially efficient trade takes always place-independent of the buyer's and the seller's degree of loss aversion.

A second simplifying assumption is that sellers can always make take-it-or-leave-it offers to the buyer. For initial contracting the distribution of bargaining power does not play a crucial role because the parties will always agree to write a contract that maximizes the joint surplus of both parties including potential losses. Therefore, the type of the concluded contract is independent of the ex ante bargaining power, only the initially specified price-which divides the expected surplus between the two parties-depends on it. The distribution of the bargaining power ex post, however, has an impact on the optimal long-term contract. As we have outlined above, the interesting effects arise because the loss-averse party can be exploited at the renegotiation stage which requires that the loss-averse party does not have too much bargaining power. In the paper we consider the extreme where the buyer is loss averse and the seller has all the bargaining power. If the buyer remains the only loss-averse party but has all the bargaining power at the renegotiation stage, materially efficient renegotiations will always take place. If, on the other hand, the seller is loss averse and the buyer can make a take-it-or-leave-it offer, materially efficient trade does not always take place in the SP-PPE under a seller employment contract with 
large acceptance set. With the buyer exploiting that the seller expects the contract to be renegotiated, it can be optimal and credible for the seller to expect that renegotiation does not always take place. Moreover, from a technical point of view, considering less extreme distributions of the bargaining power at the renegotiation stage—say, each party can make a take-it-or-leave-it offer with some probability-would increase the number of cases tremendously. In this case, expectations regarding the outcome of renegotiation would depend not only on the default outcome but also on which party is allowed to make the renegotiation offer.

Additionally, we consider a model with only three types of ex post outcomes. In particular, gains from materially efficient trade are independent of the state of nature. Due to this assumption, the optimal employment contract eliminates all variations in values and payments and therefore is globally optimal. The optimality of an employment contract with large acceptance set, however, does not rely on the fact that it eliminates all losses as it is the case in our simple setup. The major advantage of an employment contract with a large acceptance set is that it leads to few fluctuations in the default outcome. In other words, our results still hold true as long as the variations in costs and valuations for the buyer-preferred, the seller-preferred, and the materially efficient service are relatively low compared to the variations in costs and values across these services.

Finally, the reader might wonder to what extent loss aversion is different from risk aversion within the expected utility framework. A risk-averse buyer would also benefit from a contract that reduces ex post variations. Nevertheless, there are some important differences between a risk-averse and an expectation-based loss-averse buyer. Suppose the buyer is risk averse with utility function $V(v-p)$, where $V(\cdot)$ is increasing and concave. In this case, it can easily be seen that the materially efficient good is always traded ex post independent of the initial longterm contract. As for the case with a loss-averse buyer, an employment contract with complete acceptance set minimizes the variations in $\hat{v}-\hat{p}$, which is desirable also for a risk-averse buyer, and in turn maximizes the ex ante surplus of the two parties. The reason for the parties to select an employment contract if the buyer is risk averse is solely to ensure optimal risk sharing. While a risk-averse buyer is always indifferent between a buyer employment contract and a seller employment contract, a loss-averse buyer evaluates these contracts differently. Therefore, if the buyer is loss averse, the parties conclude an employment contract not only in order to reduce the risk the buyer is exposed to, but also to ensure materially efficient trade ex post.

We consider our model as a first step in gaining insights regarding optimal long-term contracting between expectation-based loss-averse parties. A more thorough analysis-which may also combine different concepts of reference point formation-is left for future research.

\section{REFERENCES}

Abeler, Johannes, Armin Falk, Lorenz Goette, and David Huffman, "Reference Points and Effort Provision," American Economic Review, 2011, 101 (2), 470-492. 
Banerji, Abhijit and Neha Gupta, "Detection, Identification, and Estimation of Loss Aversion: Evidence from an Auction Experiment," American Economic Journal: Microeconomics, 2014, 6 (1), 91-133.

Bartling, Björn and Klaus M. Schmidt, "Reference Points, Social Norms, and Fairness in Contract Renegotiations," 2013. mimeo, available at SSRN: http://ssrn.com/abstract=2123387.

_, Leif Brandes, and Daniel Schunk, "Expectations as Reference Points: Field Evidence from Professional Soccer Subjects in a Competitive, High-Stakes Environment," 2013. mimeo, available at SSRN: http://ssrn.com/abstract=2053180.

Bolton, Patrick and Mathias Dewatripont, Contract Theory, Cambridge, Massachusetts: MIT Press, 2005.

Coase, Ronald H., "The Nature of the Firm,” Economica, 1937, 4, 386-405.

_ , "The Problem of Social Cost," Journal of Law and Economics, 1960, 3, 1-44.

Crawford, Vincent P. and Juanjuan Meng, "New York City Cab Drivers' Labor Supply Revisited: Reference-Dependent Preferences with RationalExpectations Targets for Hours and Income," American Economic Review, 2011, 101 (5), 1912-1932.

Daido, Kohei and Takeshi Murooka, "Loss Aversion, Stochastic Compensation, and Team Incentives," 2013. Working Paper, University of California-Berkeley.

_, Kimiyuki Morita, Takeshi Murooka, and Hiromasa Ogawa, "Task Assignment under Agent Loss Aversion," Economics Letters, 2013, 121 (1), 35-38.

de Meza, David and David C. Webb, "Incentive Design under Loss Aversion," Journal of the European Economic Association, 2007, 5 (1), 66-92.

Eisenhuth, Roland, "Reference Dependent Mechanism design," 2012. Working Paper, Northwestern University.

Ericson, Keith M. Marzilli and Andreas Fuster, "Expectations as Endowments: Evidence on Reference-Dependent Preferences from Exchange and Valuation Experiments," Quarterly Journal of Economics, 2011, 126 (4), 1879-1907.

Fehr, Ernst, Christian Zehnder, and Oliver Hart, "Contracts, reference points, and competition-behavioral effects of the fundamental transformation," Journal of the European Economic Association, 2009, 7 (2-3), 561-572.

_ , Oliver Hart, and Christian Zehnder, "Contracts as Reference Points-Experimental Evidence," American Economic Review, 2011, 101 (2), 493-525.

$\ldots, \ldots$, and _ , "How Do Informal Agreements and Renegotiation Shape Contractual Reference Points?," Working Paper 17545, National Bureau of Economic Research October 2011.

Gill, David and Victoria Prowse, "A Structural Analysis of Disappointment Aversion in a Real Effort Competition," American Economic Review, 2012, 102 (1), 469-503.

Grossman, Sanford J. and Oliver D. Hart, "The Costs and Benefits of Ownership: A Theory of Vertical and Lateral Integration," Journal of Political Economy, 1986, 94 (4), 691-719.

Grout, Paul A., "Investment and Wages in the Absence of Binding Contracts: A Nash Bargaining Approach," Econometrica, 1984, 52 (2), 449-460.

Guasch, J. Luis, Granting and Renegotiating Infrastructure Contracts. Doing it Right, Washington, D.C.: World Bank Institute, 2004.

Hahn, Jong-Hee, Jinwoo Kim, Sang-Hyun Kim, and Jihong Lee, "Screening Loss Averse Consumers," 2012. Working Paper, Seoul National University. 
Halonen-Akatwijuka, Maija and Oliver Hart, "More is Less: Why Parties May Deliberately Write Incomplete Contracts,” 2013. mimeo.

Hart, Oliver, "Hold-up, Asset Ownership, and Reference Points," Quarterly Journal of Economics, 2009, 124 (1), 267-300.

_ , "Noncontractible Investments and Reference Points," Games, 2013, 4 (3), 437-456.

- and Bengt Holmstrom, "A Theory of Firm Scope," Quarterly Journal of Economics, 2010, $125(2), 483-513$.

_ and John Moore, "Incomplete Contracts and Renegotiation," Econometrica, 1988, 56 (4), 755-785.

_ and _ , "Property Rights and the Nature of the Firm," Journal of Political Economy, 1990, 98 (6), 1119-1158.

_ and _, "Incomplete Contracts and Ownership: Some New Thoughts," American Economic Review, 2007, 97 (2), 182-186.

_ and _, "Contracts as Reference Points," Quarterly Journal of Economics, 2008, 123 (1), $1-48$.

Heidhues, Paul and Botond Köszegi, "Competition and Price Variation When Consumers Are Loss Averse," American Economic Review, 2008, 98 (4), 1245-1268.

_ and _ , "Regular prices and sales," Theoretical Economics, 2014, 9 (1), 217-251.

Herweg, Fabian, "The Expectation-Based Loss-Averse Newsvendor," Economics Letters, 2013, 120 (3), 429-432.

- and Klaus M. Schmidt, "Loss Aversion and Inefficient Renegotiation," 2013. CESifo Working Paper Series No. 4031.

- and Konrad Mierendorff, "Uncertain Demand, Consumer Loss Aversion, and Flat-Rate Tariffs," Journal of the European Economic Association, 2013, 11 (2), 399-432.

_ , Daniel Müller, and Philipp Weinschenk, "Binary Payment Schemes: Moral Hazard and Loss Aversion,” American Economic Review, 2010, 100 (5), 2451-2477.

Hoppe, Eva I. and Patrick W. Schmitz, "Can contracts solve the hold-up problem? Experimental evidence," Games and Economic Behavior, 2011, 73 (1), 186-199.

Karle, Heiko, "Creating Attachment through Advertising: Loss Aversion and Pre-Purchase Information," 2013. Working Paper, ETH Zurich.

- and Martin Peitz, "Competition under Consumer Loss Aversion," RAND Journal of Economics, forthcoming.

_ , Georg Kirchsteiger, and Martin Peitz, "Loss Aversion and Consumption Choice: Theory and Experimental Evidence," 2012. mimeo.

Köszegi, Botond and Matthew Rabin, “A Model of Reference-Dependent Preferences," Quarterly Journal of Economics, 2006, 121 (4), 1133-1165.

_ and _, "Reference-Dependent Risk Attitudes," American Economic Review, 2007, 97 (4), 1047-1073.

_ and _ , "Reference-Dependent Consumption Plans," American Economic Review, 2009, 99 (3), 909-936.

Klein, Benjamin, Robert G. Crawford, and Armen A. Alchian, "Vertical Integration, Appropriable Rents, and the Competitive Contracting Process," Journal of Law and Economics, 1978, 21 (2), 297-326. 
List, John A., "Does Market Experience Eliminate Market Anomalies? The Case of Exogenous Market Experience," American Economic Review, 2011, 101 (3), 313-317.

Macera, Rosario, "Intertemporal Incentives with Expectation-Based Reference-Dependent Preferences," 2011. mimeo.

Maskin, Eric, "Nash equilibrium and welfare optimality," Review of Economic Studies, 1999, $66,23-38$.

Mori, Yusuke, "A Formal Behavioral Model of Firm Boundaries: Why Does Authority Relation Mitigate Ex Post Adaption Problems?," 2012. mimeo, Hitotsubashi University.

Nöldeke, Georg and Klaus M. Schmidt, "Option Contracts and Renegotiation: A Solution to the Hold-up Problem,” RAND Journal of Economics, 1995, 26 (2), 163-179.

Rosato, Antonio, "Selling Substitute Goods to Loss-Averse Consumers: Limited Availability, Bargains and Rip-offs," 2013. Working Paper, University of California-Berkeley.

Simon, Herbert A., "A Formal Theory of the Employment Relationship," Econometrica, 1951, 19 (3), 293-305.

Williamson, Oliver E., Markets and Hierachies: Analysis and Antitrust Implications, New York: Free Press, 1975.

_ , "Transaction-Cost Economics: The Governance of Contractual Relations," Journal of Law and Economics, 1979, 22 (2), 233-261.

_, The Economic Institutions of Capitalism, New York: Free Press, 1985. 


\section{A. Proofs of Propositions And LEMmas}

Proof of Proposition 1. The proof proceeds in two steps: First, we show that both sellers staying in the market is incompatible with SP-PE. The second step, which establishes that the buyer's expectations depending on the state of the world are (generically) incompatible with SP-PE, is deferred to Appendix B.

Suppose that there is a SP-PE with both sellers staying in the market. For staying in the market to be a mutual best response for both sellers at date 2, expected profits for each seller from sequentially rational behavior at date 4 given the buyer's expectations must be at least $\pi>0$. This, in turn, implies that the buyer must expect spot contracting in some state $\theta \in \Theta$ to result in seller 1 offering the delivery of some service $x^{\prime}$ at price $p^{\prime}>c\left(x^{\prime}, \theta\right)$ and himself accepting that offer with strictly positive probability. Then, however, seller 2 , whom the buyer in state $\theta \in \Theta$ must expect either to make the same offer as seller 1 or to make an offer that the buyer does not accept, could profitably deviate from the buyer's expectation by offering service $x^{\prime}$ at price $p^{\prime}-\varepsilon$ with $\varepsilon>0$. This offer is accepted by the buyer with certainty and results in strictly higher profits for seller $j$ because $p^{\prime}-\varepsilon-c\left(x^{\prime}, \theta\right)>\max \left\{0,\left[p^{\prime}-c\left(x^{\prime}, \theta\right)\right] / 2\right\}$ for $\varepsilon$ sufficiently small. This, however, implies that any SP-PE that has both sellers staying in the market has to be associated with zero profits for both sellers, a contradiction.

Proof of Lemma 2. Let

$$
\mathbf{1}_{\{k \rightarrow j\}}= \begin{cases}1 & \text { if } \hat{v}_{k}=v_{j} \\ 0 & \text { otherwise }\end{cases}
$$

denote an indicator function which takes the value one if the buyer expects default outcome $\left(v_{k}, c_{k}\right), k \in\{0, L, H\}$, to be renegotiated to outcome $\left(v_{j}, c_{j}\right), j \in\{0, L, H\}$, and which takes the value zero if the buyer expects default outcome $\left(v_{k}, c_{k}\right)$ not to be renegotiated to outcome $\left(v_{j}, c_{j}\right)$. By Lemma $1, \sum_{j \in\{0, L, H\}} \mathbf{1}_{\{k \rightarrow j\}}=1$. Furthermore, let $\hat{p}_{\{k \rightarrow j\}}$ denote the buyer's expectation regarding the trade price in case that default outcome $\left(v_{k}, c_{k}\right)$ to be renegotiated to outcome $\left(v_{j}, c_{j}\right)$. With this notation, if the buyer holds overall expectations $\Lambda$, his utility from obtaining value $v$ at price $p$ is

$$
U(v, \bar{p} \mid \Lambda)=v-p-\lambda \sum_{k \in\{0, L, H\}} \sum_{j \in\{0, L, H\}} Q_{k} \mathbf{1}_{\{k \rightarrow j\}}\left(\left[v_{j}-v\right]^{+}+\left[p-\hat{p}_{\{k \rightarrow j\}}\right]^{+}\right) .
$$

The proof, which proceeds by contradiction, is organized in two steps. First, we show that $\mathbf{1}_{\{0 \rightarrow 0\}}=1$ is incompatible with SP-PE, i.e., in any SP-PE a worthless default outcome is renegotiated to the provision of a valuable service. Second, we show that $\mathbf{1}_{\{k \rightarrow 0\}}=1$ is incompatible with SP-PE for $k \in\{L, H\}$, i.e., it will never happen in a SP-PE that a valuable default outcome is renegotiated to the provision of a worthless service.

(i) Suppose there exists a SP-PE in which the buyer expects the worthless outcome not to be renegotiated, i.e., $\mathbf{1}_{\{0 \rightarrow 0\}}=1$ and $\hat{p}_{\{0 \rightarrow 0\}}=\bar{p}$. In case of the realization of a worthless default 
outcome, suppose the seller offers materially efficient renegotiations at price $p^{R}>0$ instead of adhering to the buyer's expectations. The buyer is willing to accept this renegotiations offer if

$$
\begin{array}{r}
U\left(v_{L}, p^{R} \mid \Lambda\right)=v_{L}-p^{R}-\lambda\left(Q_{H} \mathbf{1}_{\{H \rightarrow H\}}+Q_{L} \mathbf{1}_{\{L \rightarrow H\}}\right)\left[v_{H}-v_{L}\right]-\lambda Q_{0}\left[p^{R}-\bar{p}\right] \\
-\lambda Q_{H}\left(\mathbf{1}_{\{H \rightarrow H\}}\left[p^{R}-\bar{p}\right]+\mathbf{1}_{\{H \rightarrow L\}}\left[p^{R}-\hat{p}_{\{H \rightarrow L\}}\right]+\mathbf{1}_{\{H \rightarrow 0\}}\left[p^{R}-\hat{p}_{\{H \rightarrow 0\}}\right]\right) \\
-\lambda Q_{L}\left(\mathbf{1}_{\{L \rightarrow H\}}\left[p^{R}-\hat{p}_{\{L \rightarrow H\}}\right]^{+}+\mathbf{1}_{\{L \rightarrow L\}}\left[p^{R}-\bar{p}\right]+\mathbf{1}_{\{L \rightarrow 0\}}\left[p^{R}-\hat{p}_{\{L \rightarrow 0\}}\right]\right)
\end{array}
$$

(at least weakly) exceeds

$$
\begin{array}{r}
U\left(v_{0}, \bar{p} \mid \Lambda\right)=v_{0}-\bar{p}-\lambda\left(Q_{H} \mathbf{1}_{\{H \rightarrow H\}}+Q_{L} \mathbf{1}_{\{L \rightarrow H\}}\right) v_{H}-\lambda\left(Q_{H} \mathbf{1}_{\{H \rightarrow L\}}+Q_{L} \mathbf{1}_{\{L \rightarrow L\}}\right) v_{L} \\
-\lambda Q_{H}\left(\mathbf{1}_{\{H \rightarrow L\}}\left[\bar{p}-\hat{p}_{\{H \rightarrow L\}}\right]+\mathbf{1}_{\{H \rightarrow 0\}}\left[\bar{p}-\hat{p}_{\{H \rightarrow 0\}}\right]\right)-\lambda Q_{L} \mathbf{1}_{\{L \rightarrow 0\}}\left[\bar{p}-\hat{p}_{\{L \rightarrow 0\}}\right], \quad \text { (A.4) }
\end{array}
$$

or, equivalently,

$$
\begin{aligned}
p^{R} & \leq \bar{p}+v_{L}+\lambda\left(Q_{H}\left(\mathbf{1}_{\{H \rightarrow H\}}+\mathbf{1}_{\{H \rightarrow L\}}\right)+Q_{L}\left(\mathbf{1}_{\{L \rightarrow H\}}+\mathbf{1}_{\{L \rightarrow L\}}\right)\right) v_{L} \\
& -\lambda\left(Q_{0}+Q_{L}\left(\mathbf{1}_{\{L \rightarrow L\}}+\mathbf{1}_{\{L \rightarrow 0\}}\right)+Q_{H}\right)\left[p^{R}-\bar{p}\right]-\lambda Q_{L} \mathbf{1}_{\{L \rightarrow H\}}\left[p^{R}-\hat{p}_{\{L \rightarrow H\}}\right]^{+}
\end{aligned}
$$

The seller's optimal price offer, $p^{R^{*}}$, makes (A.5) just hold with equality. Since $\hat{p}_{L \rightarrow H}>\bar{p}$, we have $\left[p^{R}-\hat{p}_{\{L \rightarrow H\}}\right]^{+}<p^{R}-\bar{p}$, it holds that

$$
p^{R^{*}}>\bar{p}+v_{L}-(1+\lambda)\left[p^{R^{*}}-\bar{p}\right] \Longleftrightarrow p^{R^{*}}>\bar{p}+\frac{v_{L}}{1+\lambda} .
$$

From (A.6), together with Assumption 1, we obtain

$$
p^{R^{*}}-c_{L}>\bar{p}
$$

i.e., the seller's profit from this deviation strictly exceeds her profit from adhering to the buyer's expectations. This, however, is incompatible with SP-PE, a contradiction.

(ii) Suppose there exists a SP-PE in which the buyer expects that a valuable default outcome will be renegotiated to a worthless service, i.e., $\mathbf{1}_{\{k \rightarrow 0\}}=1$ and $\hat{p}_{\{k \rightarrow 0\}}<\bar{p}$ for $k \in\{L, H\}$ ). Moreover, from step (i), we have $\mathbf{1}_{\{0 \rightarrow 0\}}=0$. For this to be consistent with SP-PE, the buyer expects to be indifferent between rejecting the seller's renegotiation offer, i.e., $U\left(v_{0}, \hat{p}_{\{k \rightarrow 0\}} \mid \Lambda\right)=$ $U\left(v_{k}, \bar{p} \mid \Lambda\right)$, where

$$
\begin{array}{r}
U\left(v_{0}, \hat{p}_{\{k \rightarrow 0\}} \mid \Lambda\right)=v_{0}-\hat{p}_{\{k \rightarrow 0\}}-\lambda\left(Q_{H} \mathbf{1}_{\{H \rightarrow H\}}+Q_{L} \mathbf{1}_{\{L \rightarrow H\}}+Q_{0} \mathbf{1}_{\{0 \rightarrow H\}}\right) v_{H} \\
-\lambda\left(Q_{H} \mathbf{1}_{\{H \rightarrow L\}}+Q_{L} \mathbf{1}_{\{L \rightarrow L\}}+Q_{0} \mathbf{1}_{\{0 \rightarrow L\}}\right) v_{L}-\lambda Q_{L} \mathbf{1}_{\{L \rightarrow 0\}}\left[\hat{p}_{\{k \rightarrow 0\}}-\hat{p}_{\{L \rightarrow 0\}}\right]^{+} \\
-\lambda Q_{H}\left(\mathbf{1}_{\{H \rightarrow L\}}\left[\hat{p}_{\{k \rightarrow 0\}}-\hat{p}_{\{H \rightarrow L\}}\right]^{+}+\mathbf{1}_{\{H \rightarrow 0\}}\left[\hat{p}_{\{k \rightarrow 0\}}-\hat{p}_{\{H \rightarrow 0\}}\right]^{+}\right) .
\end{array}
$$

In case of the realization of default outcome $\left(v_{k}, c_{k}\right)$, suppose the seller offers materially efficient renegotiations at price $p^{R}$ instead of adhering to the buyer's expectations and offering the provision of a worthless service. Since default outcome $v_{k}$ is itself valuable, i.e., $k \in\{L, H\}$, provision of the materially efficient service has to come along with a (weak) 
price reduction, i.e., $p^{R} \leq \bar{p}$. For the buyer to accept the seller's renegotiation offer, we must have $U\left(v_{L}, p^{R} \mid \Lambda\right) \geq U\left(v_{k}, \bar{p} \mid \Lambda\right)$, where

$$
\begin{aligned}
& U\left(v_{L}, p^{R} \mid \Lambda\right)=v_{L}-p^{R}-\lambda\left(Q_{H} \mathbf{1}_{\{H \rightarrow H\}}+Q_{L} \mathbf{1}_{\{L \rightarrow H\}}+Q_{0} \mathbf{1}_{\{0 \rightarrow H\}}\right)\left[v_{H}-v_{L}\right] \\
& -\lambda Q_{L} \mathbf{1}_{\{L \rightarrow 0\}}\left[p^{R}-\hat{p}_{\{L \rightarrow 0\}}\right]^{+}-\lambda Q_{H}\left(\mathbf{1}_{\{H \rightarrow L\}}\left[p^{R}-\hat{p}_{\{H \rightarrow L\}}\right]^{+}+\mathbf{1}_{\{H \rightarrow 0\}}\left[p^{R}-\hat{p}_{\{H \rightarrow 0\}}\right]^{+}\right) .
\end{aligned}
$$

The seller's most profitable price to offer in renegotiations, $p^{R^{*}}$, makes the buyer just indifferent between accepting and rejecting the seller's offer, i.e., $U\left(v_{L}, p^{R^{*}} \mid \Lambda\right)=U\left(v_{k}, \bar{p} \mid \Lambda\right)$. Then, with $U\left(v_{L}, p^{R^{*}} \mid \Lambda\right)=U\left(v_{0}, \hat{p}_{\{k \rightarrow 0\}} \mid \Lambda\right)$, we have

$$
\begin{aligned}
& p^{R^{*}}=\hat{p}_{\{L \rightarrow 0\}}+ v_{L} \\
&+\lambda\left(Q_{H}\left(\mathbf{1}_{\{H \rightarrow H\}}+\mathbf{1}_{\{H \rightarrow L\}}\right)+Q_{L}\left(\mathbf{1}_{\{L \rightarrow H\}}+\mathbf{1}_{\{L \rightarrow L\}}\right)+Q_{0}\left(\mathbf{1}_{\{0 \rightarrow H\}}+\mathbf{1}_{\{0 \rightarrow L\}}\right)\right) v_{L} \\
& \quad-\lambda Q_{L} \mathbf{1}_{\{L \rightarrow 0\}}\left(\left[p^{R}-\hat{p}_{\{L \rightarrow 0\}}\right]^{+}-\left[\hat{p}_{\{k \rightarrow 0\}}-\hat{p}_{\{L \rightarrow 0\}}\right]^{+}\right) \\
&-\lambda Q_{H} \mathbf{1}_{\{H \rightarrow L\}}\left(\left[p^{R}-\hat{p}_{\{H \rightarrow L\}}\right]^{+}-\left[\hat{p}_{\{k \rightarrow 0\}}-\hat{p}_{\{H \rightarrow L\}}\right]^{+}\right) \\
& \quad-\lambda Q_{H} \mathbf{1}_{\{H \rightarrow 0\}}\left(\left[p^{R}-\hat{p}_{\{H \rightarrow 0\}}\right]^{+}-\left[\hat{p}_{\{k \rightarrow 0\}}-\hat{p}_{\{H \rightarrow 0\}}\right]^{+}\right) .
\end{aligned}
$$

Note that (A.10) requires $p^{R^{*}}>\hat{p}_{\{k \rightarrow 0\}}$. Then, for $\tilde{p} \in\left\{\hat{p}_{\{L \rightarrow 0\}}, \hat{p}_{\{H \rightarrow L\}}, \hat{p}_{\{H \rightarrow 0\}}\right\}$, we have $\left[p^{R^{*}}-\tilde{p}\right]^{+}-\left[\hat{p}_{\{k \rightarrow 0\}}-\tilde{p}\right]^{+}=p^{R^{*}}-\hat{p}_{\{k \rightarrow 0\}}$ if $\hat{p}_{\{k \rightarrow 0\}} \geq \tilde{p}$ and $\left[p^{R^{*}}-\tilde{p}\right]^{+}-\left[\hat{p}_{\{k \rightarrow 0\}}-\tilde{p}\right]^{+}<$ $p^{R^{*}}-\hat{p}_{\{k \rightarrow 0\}}$ if $\hat{p}_{\{k \rightarrow 0\}}<\tilde{p}$. From (A.10) it then follows that

$$
p^{R^{*}}>\hat{p}_{\{L \rightarrow 0\}}+v_{L}-\lambda\left(Q_{L} \mathbf{1}_{\{L \rightarrow 0\}}+\lambda Q_{H}\left(\mathbf{1}_{\{H \rightarrow L\}}+\mathbf{1}_{\{H \rightarrow 0\}}\right)\right)\left(p^{R^{*}}-\hat{p}_{\{k \rightarrow 0\}}\right),
$$

which, in turn, implies

$$
p^{R^{*}}>\hat{p}_{\{L \rightarrow 0\}}+\frac{v_{L}}{1+\lambda} .
$$

Together with Assumption 1, (A.12) implies

$$
p^{R^{*}}-c_{L}>\hat{p}_{\{L \rightarrow 0\}}
$$

i.e., this deviation is indeed more profitable for the seller than adhering to the buyer's expectations. This, however, is incompatible with SP-PE, a contradiction.

Proof of Corollary 1. The proof makes use of the notation introduced in the proof of Lemma 2. Suppose the buyer holds expectations $\Lambda$ potentially consistent with SP-PE, i.e., $\mathbf{1}_{\{0 \rightarrow 0\}}=$ $\mathbf{1}_{\{L \rightarrow 0\}}=\mathbf{1}_{\{H \rightarrow 0\}}=0$. The proof proceeds in two steps: First, we show that in case of a worthless default outcome it is never profitable for the seller to deviate from the buyer's expectations by not offering renegotiations. Second, in case of a valuable default outcome it is never profitable for the seller to deviate from the buyer's expectations by offering renegotiations to the provision of a worthless service. 
(i) Suppose a worthless default outcome has been realized. The buyer's utility from this default outcome is

$$
\begin{aligned}
& U\left(v_{0}, \bar{p} \mid \Lambda\right)=v_{0}-\bar{p}-\lambda\left(Q_{0} \mathbf{1}_{\{0 \rightarrow H\}}+Q_{L} \mathbf{1}_{\{L \rightarrow H\}}+Q_{H} \mathbf{1}_{\{H \rightarrow H\}}\right) v_{H} \\
& \quad-\lambda\left(Q_{0} \mathbf{1}_{\{0 \rightarrow L\}}+Q_{L} \mathbf{1}_{\{L \rightarrow L\}}+Q_{H} \mathbf{1}_{\{H \rightarrow L\}}\right) v_{L}-\lambda Q_{H} \mathbf{1}_{\{H \rightarrow L\}}\left[\bar{p}-\hat{p}_{\{H \rightarrow L\}}\right] .
\end{aligned}
$$

On the other hand, the buyer's utility from accepting materially efficient renegotiations at price $p^{R}>\bar{p}$ is

$$
\begin{aligned}
& U\left(v_{L}, p^{R} \mid \Lambda\right)= v_{L}-p^{R}-\lambda\left(Q_{0} \mathbf{1}_{\{0 \rightarrow H\}}+Q_{L} \mathbf{1}_{\{L \rightarrow H\}}+Q_{H} \mathbf{1}_{\{H \rightarrow H\}}\right)\left(v_{H}-v_{L}\right) \\
&-\left(\lambda Q_{H} \mathbf{1}_{\{H \rightarrow H\}}+Q_{L} \mathbf{1}_{\{L \rightarrow L\}}\right)\left[p^{R}-\bar{p}\right] \\
&-\lambda Q_{H} \mathbf{1}_{\{H \rightarrow L\}}\left[p^{R}-\hat{p}_{\{H \rightarrow L\}}\right]-\lambda Q_{L} \mathbf{1}_{\{L \rightarrow H\}}\left[p^{R}-\hat{p}_{\{L \rightarrow H\}}\right]^{+} \\
&-\lambda Q_{0} \mathbf{1}_{\{0 \rightarrow L\}}\left[p^{R}-\hat{p}_{\{0 \rightarrow L\}}\right]^{+}-\lambda Q_{0} \mathbf{1}_{\{0 \rightarrow H\}}\left[p^{R}-\hat{p}_{\{0 \rightarrow H\}}\right]^{+}
\end{aligned}
$$

Proceeding in exact analogy to part the proof of (i) of Lemma 2 allows to show that the latter offer is strictly more profitable for the seller than the former.

(ii) Suppose a valuable default outcome $v_{k}, k \in\{L, H\}$, has been realized. The most profitable renegotiation offer the seller can make that involves provision of a worthless service comprises a price $p_{0}^{R^{*}}$ that equates $U\left(v_{k}, \bar{p} \mid \Lambda\right)$ and

$$
\begin{aligned}
& U\left(v_{0}, p_{0}^{R^{*}} \mid \Lambda\right)=v_{0}-p_{0}^{R^{*}}-\lambda\left(Q_{0} \mathbf{1}_{\{0 \rightarrow H\}}+Q_{L} \mathbf{1}_{\{L \rightarrow H\}}+Q_{H} \mathbf{1}_{\{H \rightarrow H\}}\right) v_{H} \\
& \quad-\lambda\left(Q_{0} \mathbf{1}_{\{0 \rightarrow L\}}+Q_{L} \mathbf{1}_{\{L \rightarrow L\}}+Q_{H} \mathbf{1}_{\{H \rightarrow L\}}\right) v_{L}-\lambda Q_{H} \mathbf{1}_{\{H \rightarrow L\}}\left[p_{0}^{R^{*}}-\hat{p}_{\{H \rightarrow L\}}\right] .
\end{aligned}
$$

Likewise, the most profitable renegotiation offer the seller can make that involves provision of materially efficient service comprises a price $p_{L}^{R^{*}}$ that equates $U\left(v_{k}, \bar{p} \mid \Lambda\right)$ and

$$
\begin{array}{r}
U\left(v_{L}, p_{L}^{R^{*}} \mid \Lambda\right)=v_{L}-p_{L}^{R^{*}}-\lambda\left(Q_{0} \mathbf{1}_{\{0 \rightarrow H\}}+Q_{L} \mathbf{1}_{\{L \rightarrow H\}}+Q_{H} \mathbf{1}_{\{H \rightarrow H\}}\right)\left(v_{H}-v_{L}\right) \\
-\lambda Q_{H} \mathbf{1}_{\{H \rightarrow L\}}\left[p_{L}^{R^{*}}-\hat{p}_{\{H \rightarrow L\}}\right]^{+} .
\end{array}
$$

Proceeding in exact analogy to part (ii) of the proof of Lemma 2 allows to show that the latter offer is strictly more profitable for the seller than the former.

Proof of Lemma 3. Considering the relevant cases of a small acceptance set and of a seller employment contract with a medium acceptance set, the proof proceeds by contradiction.

Consider an initial contract $C$ with a small acceptance set, i.e., $|\mathcal{A}|<(n+1) / 2$. Suppose $\hat{v}_{0}=$ $v_{L}, \hat{v}_{L}=v_{H}$, and $\hat{p}_{0} \leq \hat{p}_{L}$. Note that $\hat{p}_{H} \leq \bar{p}<\hat{p}_{0}$. In equilibrium, in case of a worthless default outcome, the buyer is indifferent between accepting the seller's renegotiation offer, which yields utility $U\left(v_{L}, \hat{p}_{0} \mid \Lambda(C)\right)=v_{L}-\hat{p}_{0}-\lambda Q_{L}\left(v_{H}-v_{L}\right)-\lambda Q_{H}\left[\hat{v}_{H}-v_{L}\right]^{+}-\lambda Q_{H}\left(\hat{p}_{0}-\hat{p}_{H}\right)$, and obtaining his default outcome, which yields utility $U(0, \bar{p} \mid \Lambda(C))=-\bar{p}-\lambda Q_{0} v_{L}-\lambda Q_{L} v_{H}-$ $\lambda Q_{H} \hat{v}_{H}-\lambda Q_{H}\left(\bar{p}-\hat{p}_{H}\right)$, such that

$$
\hat{p}_{0}=\bar{p}+\frac{\left(1+\lambda\left(Q_{0}+Q_{L}\right)\right) v_{L}+\lambda Q_{H} \hat{v}_{H}-\lambda Q_{H}\left[\hat{v}_{H}-v_{L}\right]^{+}}{1+\lambda Q_{H}} .
$$


Likewise, in equilibrium, in case of a materially efficient default outcome, the buyer is indifferent between accepting the seller's renegotiation offer, which yields utility $U^{B}\left(v_{H}, \hat{p}_{L} \mid \Lambda(C)\right)=$ $v_{H}-\hat{p}_{L}-\lambda Q_{0}\left(\hat{p}_{L}-\hat{p}_{0}\right)-\lambda Q_{H}\left(\hat{p}_{L}-\hat{p}_{H}\right)$, and obtaining his default outcome, which yields utility $U^{B}\left(v_{L}, \bar{p} \mid \Lambda(C)\right)=v_{L}-\bar{p}-\lambda Q_{L}\left(v_{H}-v_{L}\right)-\lambda Q_{H}\left[\hat{v}_{H}-v_{L}\right]^{+}-\lambda Q_{H}\left(\bar{p}-\hat{p}_{H}\right)$, such that

$$
\hat{p}_{L}=\bar{p}-\frac{\lambda Q_{0}}{1-\lambda\left(Q_{0}+Q_{H}\right)}\left(\hat{p}_{0}-\bar{p}\right)+\frac{\left(1+\lambda Q_{L}\right)\left(v_{H}-v_{L}\right)+\lambda Q_{H}\left[\hat{v}_{H}-v_{L}\right]^{+}}{1+\lambda\left(Q_{0}+Q_{H}\right)} .
$$

Combining (A.18) and (A.19) reveals that

$$
\hat{p}_{0} \leq \hat{p}_{L} \Longleftrightarrow 0 \leq\left(v_{H}-2 v_{L}\right)\left(1+\lambda Q_{L}\right)-\lambda Q_{0} v_{L}+2 \lambda Q_{H}\left[\hat{v}_{H}-v_{L}\right]^{+}-\lambda Q_{H} \hat{v}_{H} .
$$

With

$$
2 \lambda Q_{H}\left[\hat{v}_{H}-v_{L}\right]^{+}-\lambda Q_{H} \hat{v}_{H}=\left\{\begin{array}{ll}
-\lambda Q_{H} \hat{v}_{H} & \text { if } \hat{v}_{H} \in\left\{0, v_{L}\right\} \\
\lambda Q_{H}\left(v_{H}-2 v_{L}\right) & \text { if } \quad \hat{v}_{H}=v_{H}
\end{array}\right\} \leq 0
$$

(A.20) cannot hold, a contradiction. Thus, expectations $\hat{p}_{0} \leq \hat{p}_{L}$ are not compatible with equilibrium.

For the case of a seller employment contract with a medium acceptance set, i.e, $E=S$ and $|\mathcal{A}| \leq(n+1) / 2$, the result follows from the above derivations by setting $Q_{H}=0$.

Proof of Lemma 4. We rule out all criss-cross expectations the buyer might possibly hold as potential equilibrium expectations by identifying profitable deviations for the seller. We begin with the case of a small acceptance set, i.e., for $|\mathcal{A}|<(n+1) / 2$. Since, by Lemma 2, a worthless service will never be provided in equilibrium, we are left with four sets of expectations that involve criss-cross renegotiations. For the sake of exposition, in the following, we suppress the dependency of $Q_{k}$ on $E$ and $|\mathcal{A}|$.

CASE 1: $\hat{v}_{0} \in\left\{v_{L}, v_{H}\right\}, \hat{v}_{L}=v_{H}, \hat{v}_{H}=v_{L}$

With $\hat{p}_{H}<\bar{p}<\hat{p}_{L}<\hat{p}_{0}$ (cf. Lemma 3), $U\left(v_{H}, \hat{p}_{L} \mid \Lambda(C)\right)=v_{H}-\hat{p}_{L}-\lambda Q_{H}\left(\hat{p}_{L}-\hat{p}_{H}\right)$ equals $U\left(v_{L}, \bar{p} \mid \Lambda(C)\right)=v_{L}-\bar{p}-\lambda Q_{0}\left(\hat{v}_{0}-v_{L}\right)-\lambda Q_{L}\left(v_{H}-v_{L}\right)-\lambda Q_{H}\left(\bar{p}-\hat{p}_{H}\right)$, or equivalently,

$$
\hat{p}_{L}=\bar{p}+\frac{\left(1+\lambda Q_{L}\right)\left(v_{H}-v_{L}\right)+\lambda Q_{0}\left(\hat{v}_{0}-v_{L}\right)}{1+\lambda Q_{H}}\left(v_{H}-v_{L}\right) .
$$

Likewise, $U\left(v_{L}, \hat{p}_{H} \mid \Lambda(C)\right)=v_{L}-\hat{p}_{H}-\lambda Q_{0}\left(\hat{v}_{0}-v_{L}\right)-\lambda Q_{L}\left(v_{H}-v_{L}\right)$ equals $U\left(v_{H}, \bar{p} \mid \Lambda(C)\right)=$ $v_{H}-\bar{p}-\lambda Q_{H}\left(\bar{p}-\hat{p}_{H}\right)$, or equivalently,

$$
\hat{p}_{H}=\bar{p}-\frac{\left(1+\lambda Q_{L}\right)+\lambda Q_{0}\left(\hat{v}_{0}-v_{L}\right)}{1+\lambda Q_{H}}\left(v_{H}-v_{L}\right) .
$$

If $\left(c_{H}-c_{L}\right)>\left[\left(v_{H}-v_{L}\right)\left(1+\lambda Q_{L}\right)+\lambda Q_{0}\left(\hat{v}_{0}-v_{L}\right)\right] /\left(1+\lambda Q_{H}\right)$, then in case of $\left(v^{D}, c^{D}\right)=$ $\left(v_{L}, c_{L}\right)$ it is strictly more profitable for the seller not to offer renegotiation than to offer renegotiation to $\left(v_{H}, c_{H}\right)$ at price $\hat{p}_{L}$. If $\left(c_{H}-c_{L}\right)<\left[\left(v_{H}-v_{L}\right)\left(1+\lambda Q_{L}\right)+\lambda Q_{0}\left(\hat{v}_{0}-v_{L}\right)\right] /\left(1+\lambda Q_{H}\right)$, 
then in case of $\left(v^{D}, c^{D}\right)=\left(v_{H}, c_{H}\right)$ it is strictly more profitable for the seller not to offer renegotiation than to offer renegotiation to $\left(v_{L}, c_{L}\right)$ at price $\hat{p}_{H}$. ॥

CASE 2: $\hat{v}_{0}=v_{H}, \hat{v}_{L}=v_{L}, \hat{v}_{H}=v_{L}$

With $\hat{p}_{H}<\bar{p}=\hat{p}_{L}<\hat{p}_{0}, U\left(v_{H}, \hat{p}_{0} \mid \Lambda(C)\right)=v_{H}-\hat{p}_{0}-\lambda Q_{H}\left(\hat{p}_{0}-\hat{p}_{H}\right)-\lambda Q_{L}\left(\hat{p}_{0}-\bar{p}\right)$ equals $U(0, \bar{p} \mid \Lambda(C))=-\bar{p}-\lambda Q_{0} v_{H}-\lambda Q_{L} v_{L}-\lambda Q_{H}\left(\bar{p}-\hat{p}_{H}\right)$, or equivalently,

$$
\hat{p}_{0}=\bar{p}+\frac{\left(1+\lambda Q_{0}\right) v_{H}+\lambda\left(Q_{L}+Q_{H}\right) v_{L}}{1+\lambda\left(Q_{L}+Q_{H}\right)}
$$

Likewise, $U^{B}\left(v_{L}, \hat{p}_{H} \mid \Lambda(C)\right)=v_{L}-\hat{p}_{H}-\lambda Q_{0}\left(v_{H}-v_{L}\right)$ equals $U^{B}\left(v_{H}, \bar{p} \mid \Lambda(C)\right)=v_{H}-\bar{p}-$ $\lambda Q_{H}\left(\bar{p}-\hat{p}_{H}\right)$, or equivalently,

$$
\hat{p}_{H}=\bar{p}-\frac{1+\lambda Q_{0}}{1+\lambda Q_{H}}\left(v_{H}-v_{L}\right)
$$

If $\left(c_{H}-c_{L}\right)<\left(v_{H}-v_{L}\right)\left(1+\lambda Q_{0}\right) /\left(1+\lambda Q_{H}\right)$, then in case of $\left(v^{D}, c^{D}\right)=\left(v_{H}, c_{H}\right)$ it is strictly more profitable for the seller not to offer renegotiation than to offer renegotiation to $\left(v_{L}, c_{L}\right)$ at price $\hat{p}_{H}$. If $\left(c_{H}-c_{L}\right)>\left(v_{H}-v_{L}\right)\left(1+\lambda Q_{0}\right) /\left(1+\lambda\left(Q_{L}+Q_{H}\right)\right)$, then in case of $\left(v^{D}, c^{D}\right)=(0,0)$ it is strictly more profitable for the seller to offer renegotiation to $\left(v_{L}, c_{L}\right)$ at price

$$
p^{R}=\bar{p}+\frac{1+\lambda}{1+\lambda\left(Q_{L}+Q_{H}\right)} v_{L} \in\left(\bar{p}, \hat{p}_{0}\right)
$$

which is accepted by the buyer because $U\left(v_{L}, p^{R} \mid \Lambda(C)\right)=v_{L}-p^{R}-\lambda Q_{0}\left(v_{H}-v_{L}\right)-\lambda Q_{H}\left(p^{R}-\right.$ $\left.\hat{p}_{H}\right)-\lambda Q_{L}\left(p^{R}-\hat{p}_{L}\right)$ equals $U(0, \bar{p} \mid \Lambda(C))=-\bar{p}-\lambda Q_{0} v_{H}-\lambda\left(Q_{L}+Q_{H}\right) v_{L}-\lambda Q_{H}\left(\bar{p}-\hat{p}_{H}\right)$, than to offer renegotiation to $\left(v_{H}, c_{H}\right)$ at price $\hat{p}_{0}$. $\|$

CASE 3: $\hat{v}_{0}=v_{H}, \hat{v}_{L}=v_{L}, \hat{v}_{H}=v_{H}$

With $\hat{p}_{L}=\hat{p}_{H}=\bar{p}<\hat{p}_{0}, U\left(v_{H}, \hat{p}_{0} \mid \Lambda(C)\right)=v_{H}-\hat{p}_{0}-\lambda\left(Q_{L}+Q_{H}\right)\left(\hat{p}_{0}-\bar{p}\right)$ equals $U(0, \bar{p} \mid \Lambda(C))=-\bar{p}-\lambda Q_{L} v_{L}-\lambda\left(Q_{0}+Q_{H}\right) v_{H}$, or equivalently,

$$
\hat{p}_{0}=\bar{p}+\frac{\left(1+\lambda\left(Q_{0}+Q_{H}\right)\right) v_{H}+\lambda Q_{L} v_{L}}{1+\lambda\left(Q_{L}+Q_{H}\right)} .
$$

If $\left(c_{H}-c_{L}\right)>\left(v_{H}-v_{L}\right)\left[1+\lambda\left(Q_{0}+Q_{H}\right)\right] /\left[1+\lambda\left(Q_{L}+Q_{H}\right)\right]$, then in case of $\left(v^{D}, c^{D}\right)=(0,0)$ it is strictly more profitable for the seller to offer renegotiation to $\left(v_{L}, c_{L}\right)$ at price

$$
p^{R}=\bar{p}+\frac{1+\lambda}{1+\lambda\left(Q_{L}+Q_{H}\right)} v_{L} \in\left(\bar{p}, \hat{p}_{0}\right)
$$

which is accepted by the buyer because $U\left(v_{L}, p^{R} \mid \Lambda(C)\right)=v_{L}-p^{R}-\lambda\left(Q_{0}+Q_{H}\right)\left(v_{H}-v_{L}\right)-$ $\lambda\left(Q_{L}+Q_{H}\right)\left(p^{R}-\hat{p}_{H}\right)$ equals $U(0, \bar{p} \mid \Lambda(C))=-\bar{p}-\lambda\left(Q_{0}+Q_{H}\right) v_{H}-\lambda Q_{L} v_{L}$, than to offer renegotiation to $\left(v_{H}, c_{H}\right)$ at price $\hat{p}_{0}$. If $\left(c_{H}-c_{L}\right)<\left(v_{H}-v_{L}\right)\left[1+\lambda\left(Q_{0}+Q_{H}\right)\right] /\left[1+\lambda\left(Q_{L}+\right.\right.$ $\left.\left.Q_{H}\right)\right]$, then in case of $\left(v^{D}, c^{D}\right)=\left(v_{L}, c_{L}\right)$ it is strictly more profitable for the seller to offer renegotiation to $\left(v_{H}, c_{H}\right)$ at price

$$
p^{R}=\bar{p}+\frac{1+\lambda\left(Q_{0}+Q_{H}\right)}{1+\lambda\left(Q_{L}+Q_{H}\right)}\left(v_{H}-v_{L}\right) \in\left(\bar{p}, \hat{p}_{0}\right),
$$


which is accepted by the buyer because $U\left(v_{H}, p^{R} \mid \Lambda(C)\right)=v_{H}-p^{R}-\lambda\left(Q_{L}+Q_{H}\right)\left(p^{R}-\hat{p}_{H}\right)$ equals $U\left(v_{L}, \bar{p} \mid \Lambda(C)\right)=v_{L}-\bar{p}-\lambda\left(Q_{0}+Q_{H}\right)\left(v_{H}-v_{L}\right)$, than not to offer renegotiation. ॥

For a medium acceptance set, i.e., for $|\mathcal{A}|=(n+1) / 2$, in the light of Lemma 2 there is only one set of expectations involving criss-cross renegotiations to consider. In case of a seller employment contract, $\left(\hat{v}_{0}, \hat{v}_{L}\right)=\left(v_{H}, v_{L}\right)$ can be ruled out in analogy to CASE 3 . The corresponding algebraic expressions are obtained by setting $Q_{H}=0$. In case of a buyer employment contract, $\left(\hat{v}_{L}, \hat{v}_{H}\right)=\left(v_{H}, v_{L}\right)$ can be ruled in analogy to CASE 1 . The corresponding algebraic expressions are obtained by setting $Q_{0}=0$.

Proof of Proposition 2. With $\hat{p}_{H}^{L L L}<\hat{p}_{L}^{L L L}=\bar{p}<\hat{p}_{0}^{L L L}$, the equilibrium price $\hat{p}_{H}^{L L L}$ in (18) is obtained by equating $U\left(v_{L}, \hat{p}_{H} \mid \Lambda^{L L L}(C)\right)=v_{L}-\hat{p}_{H}^{L L}$ and $U\left(v_{H}, \bar{p} \mid \Lambda^{L L L}(C)\right)=v_{H}-\bar{p}-$ $\lambda Q_{H}\left(\bar{p}-\hat{p}_{H}^{L L L}\right)$. Likewise, the equilibrium price $\hat{p}_{0}^{L L L}$ in (17) is obtained by equating $U\left(v_{L}, \hat{p}_{0} \mid\right.$ $\left.\Lambda^{L L L}(C)\right)=v_{L}-\hat{p}_{0}^{L L L}-\lambda Q_{H}\left(\hat{p}_{0}^{L L L}-\hat{p}_{H}^{L L L}\right)-\lambda Q_{L}\left(\hat{p}_{0}^{L L L}-\bar{p}\right)$ and $U\left(0, \bar{p} \mid \Lambda^{L L L}(C)\right)=$ $-\bar{p}-\lambda v_{L}-\lambda Q_{H}\left(\bar{p}-\hat{p}_{H}^{L L L}\right)$. By Corollary 1, deviations in form of offering provision of a useless service are never profitable for the seller.

If $\left(v^{D}(C), c^{D}(C)\right)=\left(v_{L}, v_{L}\right)$, then the highest price $p^{R}>\bar{p}$ at which the buyer would accept renegotiation to $\left(v_{H}, c_{H}\right)$ is below $\hat{p}_{0}^{L L L}$ and equates $U\left(v_{H}, p^{R} \mid \Lambda^{L L L}(C)\right)=v_{H}-p^{R}-$ $\lambda Q_{L}\left(p^{R}-\bar{p}\right)-\lambda Q_{H}\left(p^{R}-\hat{p}_{H}^{L L L}\right)$ and $U\left(v_{L}, \bar{p} \mid \Lambda^{L L L}(C)\right)=v_{L}-\bar{p}-\lambda Q_{H}\left(\bar{p}-\hat{p}_{H}^{L L L}\right)$, i.e., $p^{R}=\bar{p}+\left(v_{H}-v_{L}\right) /\left[1+\lambda\left(Q_{L}+Q_{H}\right)\right]$. Offering this deviation, however, is not profitable for the seller because $p^{R}-\bar{p}=\left(v_{H}-v_{L}\right) /\left[1+\lambda\left(Q_{L}+Q_{H}\right)\right]<c_{H}-c_{L}$.

If $\left(v^{D}(C), c^{D}(C)\right)=(0,0)$, then the highest price $p^{R}>\bar{p}$ at which the buyer would accept renegotiation to $\left(v_{H}, c_{H}\right)$ is higher than $\hat{p}_{0}^{L L L}$ and equates $U\left(v_{H}, p^{R} \mid \Lambda^{L L L}(C)\right)=v_{H}-p^{R}-$ $\lambda Q_{H}\left(p^{R}-\hat{p}_{H}^{L L L}\right)-\lambda Q_{L}\left(p^{R}-\bar{p}\right)-\lambda Q_{0}\left(p^{R}-\hat{p}_{0}^{L L L}\right)$ and $U\left(0, \bar{p} \mid \Lambda^{L L L}(C)\right)=-\bar{p}-\lambda v_{L}-$ $\lambda Q_{H}\left(\bar{p}-\hat{p}_{H}^{L L L}\right)$, i.e., $p^{R}=\hat{p}_{0}^{L L L}+\left[v_{H}-v_{L}\right] /(1+\lambda)$. Offering this deviation, however, is not profitable for the seller because $p^{R}-\hat{p}_{0}^{L L L}=\left(v_{H}-v_{L}\right) /(1+\lambda)<c_{H}-c_{L}$.

If $\left(v^{D}(C), c^{D}(C)\right)=\left(v_{H}, c_{H}\right)$, then not offering renegotiations is not profitable because $\bar{p}-\hat{p}_{H}^{L L L}=\left(v_{H}-v_{L}\right) /\left[1+\lambda Q_{H}\right]<c_{H}-c_{L}$.

Proof of Proposition 3. We prove the result in several steps. For the sake of exposition, in the following, we suppress the dependency of $Q_{k}$ on $E$ and $|\mathcal{A}|$.

STEP 1: $\Lambda^{L L H}$ IS CONSISTENT WITH SP-PE IF AND ONLY IF $\lambda \geq \tilde{\lambda}_{E, \mathcal{A}}$. With $\hat{p}_{H}^{L L H}=\hat{p}_{L}^{L L H}=\bar{p}<\hat{p}_{0}^{L L H}$, the equilibrium price $\hat{p}_{0}^{L L H}$ in (21) is obtained by equating $U\left(v_{L}, \hat{p}_{0} \mid \Lambda^{L L H}(C)\right)=v_{L}-\hat{p}_{0}^{L H}-\lambda Q_{H}\left(v_{H}-v_{L}\right)-\lambda\left(Q_{L}+Q_{H}\right)\left(\hat{p}_{0}^{L L H}-\bar{p}\right)$ and $U\left(0, \bar{p} \mid \Lambda^{L L H}(C)\right)=-\bar{p}-\lambda\left(Q_{0}+Q_{L}\right) v_{L}-\lambda Q_{H} v_{H}$. By Corollary 1 , deviations in form of offering provision of a useless service are never profitable for the seller.

If $\left(v^{D}(C), c^{D}(C)\right)=\left(v_{L}, v_{L}\right)$, then the highest price $p^{R}>\bar{p}$ at which the buyer would accept renegotiation to $\left(v_{H}, c_{H}\right)$ is below $\hat{p}_{0}^{L L H}$ and equates $U\left(v_{H}, p^{R} \mid \Lambda^{L L H}(C)\right)=v_{H}-p^{R}-\lambda\left(Q_{L}+\right.$ $\left.Q_{H}\right)\left(p^{R}-\bar{p}\right)$ and $U\left(v_{L}, \bar{p} \mid \Lambda^{L L H}(C)\right)=v_{L}-\bar{p}-\lambda Q_{H}\left(v_{H}-v_{L}\right)$, i.e. $p^{R}=\bar{p}+\left(v_{H}-v_{L}\right)(1+$ $\left.\lambda Q_{H}\right) /\left[1+\lambda\left(Q_{L}+Q_{H}\right)\right]$. Offering this deviation, however, is not profitable for the seller because $p^{R}-\bar{p}=\left(v_{H}-v_{L}\right)\left(1+\lambda Q_{H}\right) /\left[1+\lambda\left(Q_{L}+Q_{H}\right)\right]<c_{H}-c_{L}$. 
If $\left(v^{D}(C), c^{D}(C)\right)=(0,0)$, then the highest price $p^{R}>\bar{p}$ at which the buyer would accept renegotiation to $\left(v_{H}, c_{H}\right)$ is higher than $\hat{p}_{0}^{L L H}$ and equates $U\left(v_{H}, p^{R} \mid \Lambda^{L L H}(C)\right)=v_{H}-p^{R}$ $\lambda\left(Q_{L}+Q_{H}\right)\left(p^{R}-\bar{p}\right)-\lambda Q_{0}\left(p^{R}-\hat{p}_{0}^{L L H}\right)$ and $U\left(0, \bar{p} \mid \Lambda^{L L H}(C)\right)=-\bar{p}-\lambda\left(Q_{0}+Q_{L}\right) v_{L}-\lambda Q_{H} v_{H}$, i.e. $p^{R}=\bar{p}+\left(\hat{p}_{0}^{L L H}-\right.$ barp $) \lambda Q_{0} /(1+\lambda)+\left[\left(1+\lambda Q_{H}\right) v_{H}+\lambda\left(Q_{0}+Q_{L}\right) v_{L}\right] /(1+\lambda)$. Offering this deviation, however, is not profitable for the seller because $p^{R}-\hat{p}_{0}^{L L H}=\left(v_{H}-v_{L}\right)[1-$ $\left.\lambda\left(Q_{0}+Q_{L}\right)\right] /(1+\lambda)<c_{H}-c_{L}$.

If $\left(v^{D}(C), c^{D}(C)\right)=\left(v_{H}, c_{H}\right)$, then the highest price $p^{R}<\bar{p}$ at which the buyer would accept renegotiation to $\left(v_{L}, c_{L}\right)$ equates $U\left(v_{L}, p^{R} \mid \Lambda^{L L H}(C)\right)=v_{L}-p^{R}-\lambda Q_{H}\left(v_{H}-v_{L}\right)$ and $U\left(v_{H}, \bar{p} \mid \Lambda^{L L H}(C)\right)=v_{H}-\bar{p}$, i.e. $p^{R}=\bar{p}-\left(1+\lambda Q_{H}\right)\left(v_{H}-v_{L}\right)$. Offering this deviation is more profitable for the seller than not to offer renegotiation if $\bar{p}-p^{R}=\left(1+\lambda Q_{H}\right)\left(v_{H}-v_{L}\right)<c_{H}-c_{L}$. Thus, $\lambda \geq \tilde{\lambda}_{E, \mathcal{A}}$ or, equivalently,

$$
\frac{c_{H}-c_{L}}{v_{H}-v_{L}} \leq 1+\lambda Q_{H}(E, \mathcal{A})
$$

is a necessary and sufficient condition for expectations $\Lambda^{L L H}$ to be consistent with SP-PE. ॥

STEP 2: NECESSARY CONDITION FOR $\Lambda^{L H H}$ TO BE CONSISTENT WITH SP-PE.

Consider expectations $\Lambda\left(C_{E, \mathcal{A}}(\bar{p})\right)=\left\{\left(v_{L}, \hat{p}_{0}^{L H H}\right),\left(v_{H}, \hat{p}_{L}^{L H H}\right),\left(v_{H}, \bar{p}\right)\right\}=\Lambda^{L H H}$. With $\hat{p}_{H}^{L H H}=\bar{p}<\hat{p}_{L}^{L H H}<\hat{p}_{0}^{L H H}$, the expected price $\hat{p}_{L}^{L H H}$ is obtained by equating $U\left(v_{H}, \hat{p}_{L}^{L H H} \mid\right.$ $\left.\Lambda^{L H H}\right)=v_{H}-\hat{p}_{L}^{L H H}-\lambda Q_{H}\left(\hat{p}_{L}^{L H H}-\bar{p}\right)$ and $U\left(v_{L}, \bar{p} \mid \Lambda^{L H H}\right)=v_{L}-\bar{p}-\lambda\left(Q_{L}+Q_{H}\right)\left(v_{H}-v_{L}\right)$, which yields

$$
\hat{p}_{L}^{L H H}=\bar{p}+\frac{1+\lambda\left(Q_{L}+Q_{H}\right)}{1+\lambda Q_{H}}\left(v_{H}-v_{L}\right) .
$$

The expected price $\hat{p}_{0}^{L H H}$ is obtained by equating $U\left(v_{L}, \hat{p}_{0}^{L H H} \mid \Lambda^{L H H}\right)=v_{L}-\hat{p}_{0}^{L H H}-\lambda\left(Q_{L}+\right.$ $\left.Q_{H}\right)\left(v_{H}-v_{L}\right)-\lambda Q_{L}\left(\hat{p}_{0}^{L H H}-\hat{p}_{L}^{L H H}\right)-\lambda Q_{H}\left(\hat{p}_{0}^{L H H}-\bar{p}\right)$ and $U\left(0, \bar{p} \mid \Lambda^{L H H}\right)=-\bar{p}-\lambda Q_{0} v_{L}-$ $\lambda\left(Q_{L}+Q_{H}\right) v_{H}$, which yields

$$
\hat{p}_{0}^{L H H}=\hat{p}_{L}^{L H H}+\left\{\frac{1+\lambda}{1+\lambda\left(Q_{L}+Q_{H}\right)} v_{L}-\left(v_{H}-v_{L}\right)\right\}
$$

In case of the materially efficient default outcome $\left(v_{L}, c_{L}\right)$ it is strictly more profitable for the seller not to offer renegotiation than to offer renegotiation to $\left(v_{H}, c_{H}\right)$ at price $\hat{p}_{L}$ if $\bar{p}-c_{L}>$ $\hat{p}_{L}-c_{H}$, or equivalently, $\left(c_{H}-c_{L}\right)>\left(v_{H}-v_{L}\right)\left[1+\lambda\left(Q_{L}+Q_{H}\right)\right] /\left(1+\lambda Q_{H}\right)$. Thus,

$$
\frac{c_{H}-c_{L}}{v_{H}-v_{L}} \leq \frac{1+\lambda\left(Q_{L}+Q_{H}\right)}{1+\lambda Q_{H}} .
$$

is a necessary condition for expectations $\Lambda^{L H H}$ to be consistent with SP-PE. II

STEP 3: NECESSARY CONDITION FOR $\Lambda^{H H H}$ TO BE CONSISTENT WITH SP-PE.

Consider expectations $\Lambda\left(C_{E, \mathcal{A}}(\bar{p})\right)=\left\{\left(v_{H}, \hat{p}_{0}^{H H H}\right),\left(v_{H}, \hat{p}_{L}^{H H H}\right),\left(v_{H}, \bar{p}\right)\right\}=: \Lambda^{H H H}$. With $\hat{p}_{H}^{H H H}=\bar{p}<\hat{p}_{L}^{H H H}<\hat{p}_{0}^{H H H}$, the equilibrium price $\hat{p}_{L}^{H H H}$ is obtained by equating $U\left(v_{H}, \hat{p}_{L}^{H H H} \mid\right.$ 
$\left.\Lambda^{H H H}\right)=v_{H}-\hat{p}_{L}^{H H H}-\lambda Q_{H}\left(\hat{p}_{L}^{H H H}-\bar{p}\right)$ and $U\left(v_{L}, \bar{p} \mid \Lambda^{H H H}\right)=v_{L}-\bar{p}-\lambda\left(v_{H}-v_{L}\right)$, which yields

$$
\hat{p}_{L}^{H H H}=\bar{p}+\frac{1+\lambda}{1+\lambda Q_{H}}\left(v_{H}-v_{L}\right) .
$$

The equilibrium price $\hat{p}_{0}^{H H H}$ is obtained by equating $U\left(v_{H}, \hat{p}_{0}^{H H H} \mid \Lambda^{H H H}\right)=v_{H}-\hat{p}_{0}^{H H H}-$ $\lambda Q_{L}\left(\hat{p}_{0}^{H H H}-\hat{p}_{L}^{H H H}\right)-\lambda Q_{H}\left(\hat{p}_{0}^{H H H}-\bar{p}\right)$ and $U\left(0, \bar{p} \mid \Lambda^{H H H}\right)=-\bar{p}-\lambda v_{H}$, which yields

$$
\hat{p}_{0}^{H H H}=\hat{p}_{L}^{H H H}+\frac{1+\lambda}{1+\lambda\left(Q_{L}+Q_{H}\right)} v_{L} .
$$

In case of the useless default outcome $(0,0)$ it is strictly more profitable for the seller to offer renegotiation to $\left(v_{L}, c_{L}\right)$ at price

$$
p^{R}=\bar{p}+\frac{\lambda Q_{L}}{\left[1+\lambda\left(Q_{L}+Q_{H}\right)\right]} \frac{(1+\lambda)}{\left(1+\lambda Q_{H}\right)}\left(v_{H}-v_{L}\right)+\frac{(1+\lambda)}{1+\lambda\left(Q_{L}+Q_{H}\right)} v_{L} \in\left(\hat{p}_{L}, \hat{p}_{H}\right),
$$

which is accepted by the buyer because $U\left(v_{L}, p^{R} \mid \Lambda^{H H H}\right)=v_{L}-p^{R}-\lambda\left(v_{H}-v_{L}\right)-\lambda Q_{L}\left(p^{R}-\right.$ $\left.\hat{p}_{L}\right)-\lambda Q_{H}\left(p^{R}-\bar{p}\right)$ equals $U\left(0, \bar{p} \mid \Lambda^{H H H}\right)$, than to offer renegotiation to $\left(v_{H}, c_{H}\right)$ at price $\hat{p}_{L}$ if $p^{R}-c_{L}>\hat{p}_{0}-c_{H}$, or equivalently, $\left(c_{H}-c_{L}\right)>\left(v_{H}-v_{L}\right)(1+\lambda) /\left(1+\lambda\left(Q_{L}+Q_{H}\right)\right)$. Thus,

$$
\frac{c_{H}-c_{L}}{v_{H}-v_{L}} \leq \frac{1+\lambda}{1+\lambda\left(Q_{L}+Q_{H}\right)}
$$

is a necessary condition for expectations $\Lambda^{H H H}$ to be consistent with SP-PE. ॥

\section{STEP 4: COMPARISON OF EXISTENCE CONDITIONS.}

The condition (A.30) for expectations $\Lambda^{L L H}$ to be consistent with SP-PE is less restrictive than the corresponding condition (A.33) for expectations $\Lambda^{L H H}$ if and only if $1+\lambda Q_{H} \geq[1+$ $\left.\lambda\left(Q_{L}+Q_{H}\right)\right] /\left(1+\lambda Q_{H}\right)$, or equivalently, $\left(1+\lambda Q_{H}\right) Q_{H} \geq Q_{L}$. Note that this latter condition holds if $Q_{H} \geq Q_{L}$, which in particular is the case for $Q_{H} \geq 1 / 2$.

The condition (A.30) for expectations $\Lambda^{L L H}$ to be consistent with SP-PE is less restrictive than the existence corresponding (A.37) for expectations $\Lambda^{H H H}$ if and only if $1+\lambda Q_{H} \geq$ $(1+\lambda) /\left[1+\lambda\left(Q_{L}+Q_{H}\right)\right]$, or equivalently, $2 Q_{H}+Q_{L}+\lambda Q_{H}\left(Q_{L}+Q_{H}\right) \geq 1$. Note that $Q_{H} \geq 1 / 2$ is sufficient for this condition to be satisfied. ॥

SteP 5: NeITHer $\Lambda^{L H H}$ NOR $\Lambda^{H H H}$ CONSTitutes THE SP-PPE. The buyer's expected utility under expectations $\Lambda^{L H H}$ and $\Lambda^{H H H}$ is

$$
E U\left(\Lambda^{L H H}\right)=v_{L}-\bar{p}-Q_{0}(1+\lambda) v_{L}+\left[Q_{H}\left(1-\lambda\left(1-Q_{H}\right)\right)-\lambda Q_{L}\left(1-Q_{H}\right)\right]\left(v_{H}-v_{L}\right)
$$

and

$$
E U\left(\Lambda^{H H H}\right)=v_{L}-\bar{p}-Q_{0}(1+\lambda) v_{L}+\left[1-(1+\lambda)\left(1-Q_{H}\right)\right]\left(v_{H}-v_{L}\right),
$$


respectively. Comparison of (22), (A.38), and (A.39) reveals that $E U\left(\Lambda^{L L H}\right) \geq$ $\max \left\{E U\left(\Lambda^{L H H}\right), E U\left(\Lambda^{H H H}\right)\right\}$ whenever all three sets of expectations are consistent with subsequently rational behavior. Thus, from STEP 4 it follows that for $Q_{H} \geq 1 / 2$ the buyer's preferred personal equilibrium is neither $\Lambda^{L H H}$ nor $\Lambda^{H H H}$.

Comparison of (19) and (A.39) reveals that $E U\left(\Lambda^{L L L}\right)>E U\left(\Lambda^{H H H}\right)$ if and only if

$$
1-(1+\lambda)\left(1-Q_{H}\right)<Q_{H} \frac{1-\lambda\left(1-Q_{H}\right)}{1+\lambda Q_{H}} \Longleftrightarrow Q_{H}\left[1-\lambda\left(1-Q_{H}\right)\right]<1-Q_{H},
$$

which clearly holds for $Q_{H}<1 / 2$.

Comparison of (19) and (A.38) reveals that $E U\left(\Lambda^{L L L}\right)>E U\left(\Lambda^{L H H}\right)$ if and only if

$$
1 \geq \frac{Q_{H}}{1-Q_{H}} \cdot \frac{1-\lambda\left(1-Q_{H}\right)}{\left(1+\lambda Q_{H}\right)} \cdot \frac{Q_{H}}{Q_{L}}
$$

Thus, $\Lambda^{L H H}$ is not the buyer's SP-PPE for $Q_{H}<1 / 2$ if $Q_{L} \geq Q_{H}$. Moreover, from the observation above in combination with STEP 4, we know $\Lambda^{L H H}$ is not the buyer's SP-PPE for $Q_{H}<1 / 2$ if $Q_{L}<Q_{H}$ because $E U\left(\Lambda^{L L H}\right) \geq E U\left(\Lambda^{L H H}\right)$. ॥

Proof of Corollary 2. Suppose for $|\mathcal{A}|<(n+1) / 2$. Part (i) is trivial. Part (ii) follows from the definition of $\tilde{\lambda}_{E, \mathcal{A}}$ in (15) together with the fact that $Q(E, \mathcal{A})=(n-1) / 2 n$ is increasing in $n$.

Proof of Proposition 4. The proofs of $i$ ) and $i i$ ) are provided in the main text with $\tilde{\lambda}_{E, \mathcal{A}}$ being determined by (A.30) with $Q_{H}=1$.

Proof of Proposition 5. The proof of this proposition is provided in the main text.

Proof of Proposition 6. In general, the desired result is established in analogy to the proofs of Propositions 2 and 3.

(i) For $E=B$, the default outcome will never be a worthless service. Therefore, when we make references to earlier established results, set $Q_{0}=0$ and ignore all derivations for the case of a worthless default outcome.

Regarding $\Lambda_{B}^{L L}$, following along the lines of the proof of Proposition 2, the renegotiated trade price $\hat{p}_{H}^{L L}$ and the buyer's expected utility $E U\left(\Lambda^{L L}\right)$ correspond to (18) and (19), respectively.

Regarding $\Lambda_{B}^{L H}$, following along the lines of STEP 1 in the proof of Proposition 3, the buyer's expected utility $E U\left(\Lambda^{L H}\right)$ and the condition for $\Lambda_{B}^{L H}$ to be consistent with SP-PE correspond to (22) and (A.30), respectively.

Regarding $\Lambda_{B}^{H H}$, following along the lines of STEP 2 in the proof of Proposition 3, the renegotiated trade price $\hat{p}_{L}^{H H}$, the buyer's expected utility $E U\left(\Lambda^{H H}\right)$, and the condition for $\Lambda_{B}^{H H}$ to be consistent with SP-PE correspond to (A.31), (A.38), and (A.33), respectively.

Finally, similar to STEP 4 and STEP 5 of Proposition 3, the following can be shown: first, the buyer's expected utility is higher under $\Lambda_{B}^{L H}$ than under $\Lambda_{B}^{L L}$ and $\Lambda_{B}^{H H}$; second, for $Q_{H} \geq 1 / 2$, 
$\Lambda_{B}^{L H}$ is consistent with SP-PE whenever $\Lambda_{B}^{H H}$ is consistent with SP-PE; third, for $Q_{H} \geq 1 / 2$, the buyer's expected utility is higher under $\Lambda_{B}^{L L}$ than under $\Lambda_{B}^{H H}$.

(ii) For $E=S$, the default outcome will never be a high-value/high-cost service. Therefore, when we make references to earlier established results, set $Q_{H}=0$ and ignore all derivations for the case of a high-value/high-cost default outcome.

Regarding $\Lambda_{S}^{L L}$, following along the lines of the proof of Proposition 2, the renegotiated trade price $\hat{p}_{0}^{L L}$ and the buyer's expected utility $E U\left(\Lambda^{L L}\right)$ correspond to (17) and (19), respectively.

Regarding $\Lambda_{S}^{L H}$, following along the lines of STEP 2 in the proof of Proposition 3, the renegotiated trade prices $\hat{p}_{L}^{L H}$ and $\hat{p}_{0}^{L H}$ and the buyer's expected utility $E U\left(\Lambda^{L H}\right)$ correspond to (A.31), (A.32), and (A.38), respectively.

Regarding $\Lambda_{S}^{H H}$, following along the lines of STEP 3 in the proof of Proposition 3, the renegotiated trade prices $\hat{p}_{L}^{H H}$ and $\hat{p}_{0}^{H H}$ and the buyer's expected utility $E U\left(\Lambda^{H H}\right)$ correspond to (A.34), (A.35), and (A.39), respectively.

Finally, it can be shown that the buyer's expected utility is higher under $\Lambda_{S}^{L L}$ than under $\Lambda_{S}^{L H}$ and $\Lambda_{S}^{H H}$.

Proof of Proposition 7. The statement follows from the Bertrand nature of seller competition at the initial stage at which long-term contract offers are made, together with Proposition 2, 3, 4, 5 and 6.

Proof of Proposition 8. First, we prove the main claim of the proposition, i.e., $C=C_{B, \mathcal{A}}(\bar{p})$ with $|\mathcal{A}|=(n+1) / 2$ maximizes the buyer's willingness to invest. Note that, for $\lambda \leq \tilde{\lambda}_{B, \mathcal{L}}(0)$, materially efficient renegotiation occurs for all $I \in\{0,1\}$ and all $\mathcal{A} \subseteq \mathcal{X}$. This holds true because by (15), we have (i) $\tilde{\lambda}_{E, \mathcal{A}}(0)<\tilde{\lambda}_{E, \mathcal{A}}(1)$ for all $\mathcal{A}$ and all $E$, and (ii) $\tilde{\lambda}_{E, \mathcal{A}}(0)$ is decreasing in $Q_{H}(E, \mathcal{A})$ and thus lowest for $Q_{H}=1$-i.e, for a buyer employment contract with large acceptance set.

Next, we show that if $\lambda \leq \tilde{\lambda}_{B, \mathcal{L}}(0)$, the threshold of investment costs is largest for a buyer employment contract with medium acceptance set. In order to cut back on notation, let

$$
\Delta_{k}:=v_{k}(1)-v_{k}(0),
$$

for $k \in\{L, H\}$. Consider a buyer employment contract with a small (or medium) acceptance set. In the following, we make use of the shortcut notation $Q_{k}(s)$ for $Q_{k}(E, \mathcal{A} \mid s)$. As argued in the main text, $\psi_{B, \mathcal{S}}(s, \lambda)$-defined by (47) —is decreasing in $s$, which holds true because

$$
\begin{aligned}
\frac{\partial \psi_{B, \mathcal{S}}(s, \lambda)}{\partial s} & =-Q_{0}^{\prime}(s)(1+\lambda) \Delta_{L}-\left(\Delta_{L}-\Delta_{H}\right) \frac{Q_{H}^{\prime}(s)-\lambda Q_{H}^{\prime}(s)+2 Q_{H} \lambda Q_{H}^{\prime}(s)}{\left(1+\lambda Q_{H}\right)^{2}} \\
& =-Q_{0}^{\prime}(s)\left[\Delta_{H} \frac{1-\lambda+2 Q_{H}}{\left(1+\lambda Q_{H}\right)^{2}}+\Delta_{L}\left(1+\lambda-\frac{1-\lambda+2 Q_{H}}{\left(1+\lambda Q_{H}\right)^{2}}\right)\right]<0 .
\end{aligned}
$$

Note that $-Q_{H}^{\prime}(s)=Q_{0}^{\prime}(s)=1 / n$. 
Thus, $\psi_{B, \mathcal{S}}(s, \lambda)$ is maximized at $s=0$, where $s=0$ corresponds to a medium acceptance set. In addition, $\psi_{B, \mathcal{S}}(s=0, \lambda)$ is larger than the threshold of investment costs, $\psi_{B, \mathcal{L}}(\lambda)$, under a buyer employment contract with a large acceptance set. From (45) and (47), it follows that

$$
\begin{aligned}
& \psi_{B, \mathcal{S}}(s=0, \lambda) \geq \psi_{B, \mathcal{L}}(\lambda) \\
\Longleftrightarrow & \frac{1}{1+\lambda} \geq Q_{H} \frac{1-\lambda+\lambda Q_{H}}{1+\lambda Q_{H}} \\
\Longleftrightarrow & 0 \geq-\left(1-Q_{H}\right)\left(1+\lambda Q_{H}+\lambda^{2} Q_{H}\right) .
\end{aligned}
$$

In order to conclude this part of the proof it remains to be shown that a seller employment contract never leads to higher investment incentives. Tedious but straightforward calculationswhich are omitted-reveal that the critical investment threshold under a seller employment contract, $\psi_{S, \mathcal{A}}(\cdot)$, is always below the threshold for a buyer employment contract with large acceptance set. Formally, $\psi_{S, \mathcal{A}}(\cdot) \leq \psi_{B, \mathcal{L}}$ for all $\mathcal{A} \subseteq \mathcal{X}$ and all $\lambda \geq 0$. Thus, for $\lambda \leq \tilde{\lambda}_{B, \mathcal{L}}(0)$, the threshold of investment costs $\psi_{B, \mathcal{S}}(s=0, \lambda)$ is the largest threshold.

To prove the second part of the proposition, it remains to be established that the set of investment costs for which the buyer invests under a buyer employment contract with a small acceptance set is increasing in his degree of loss aversion if $|\mathcal{A}|$ is sufficiently large. By (47) it holds that

$$
\begin{aligned}
\frac{\partial \psi_{B, \mathcal{S}}(k, \lambda)}{\partial \lambda} & =-Q_{H} \frac{-\left(1-Q_{H}\right)\left(1+\lambda Q_{H}\right)-Q_{H}\left(1-\lambda\left(1-Q_{H}\right)\right)}{\left(1+\lambda Q_{H}\right)^{2}}\left(\Delta_{L}-\Delta_{H}\right)-Q_{0} \Delta_{L} \\
& =-\frac{Q_{H}}{\left(1+\lambda Q_{H}\right)^{2}}\left[\Delta_{H}+\Delta_{L}\left(\frac{Q_{0}}{Q_{H}}\left(1+\lambda Q_{H}\right)^{2}-1\right)\right] \\
& =-\frac{Q_{H}}{\left(1+\lambda Q_{H}\right)^{2}}\left[\Delta_{H}+\Delta_{L}\left(\frac{s}{n-1-s}\left(1+\lambda Q_{H}\right)^{2}-1\right)\right] .
\end{aligned}
$$

From the above derivative, we immediately obtain

$$
\left.\frac{\partial \psi_{B, \mathcal{S}}(s, \lambda)}{\partial \lambda}\right|_{s=0}=\frac{Q_{H}}{\left(1+\lambda Q_{H}\right)^{2}}\left(\Delta_{L}-\Delta_{H}\right)>0
$$

and

$$
\left.\frac{\partial \psi_{B, \mathcal{S}}(s, \lambda)}{\partial \lambda}\right|_{s=\frac{n-1}{2}}=-\frac{Q_{H}}{\left(1+\lambda Q_{H}\right)^{2}}\left(\Delta_{H}+\Delta_{L}\left(2 \lambda Q_{H}+\lambda^{2} Q_{H}^{2}\right)\right)<0 .
$$

Moreover,

$$
\frac{\partial^{2} \psi_{B, \mathcal{S}}(s, \lambda)}{\partial s \partial \lambda}=-\frac{1}{n}\left(\left(\Delta_{L}-\Delta_{H}\right) \frac{1-\lambda Q_{H}}{\left(1+\lambda Q_{H}\right)^{3}}+\Delta_{L}\right)<0 .
$$

This proves the second part of the proposition.

\section{B. REMARKS ON GENERICITY}

Proof of Proposition 1. Step 2: We establish that the buyer's expectations depending on the state of the world are generically incompatible with SP-PE. 
Suppose the buyer expects only one seller to stay in the market. As we have argued in the text, it is incompatible with SP-PE for the buyer to expect to obtain a zero value in all states of the world. It remains to establish that, for given expectations $\Lambda(\varnothing)$ with $\hat{v}(\theta, \varnothing) \neq 0$ for some $\theta \in \Theta$, a monopoly seller (generically) will offer one and the same spot contract in each state of the world. According to (7), the buyer's utility from accepting a spot contract resulting in value $v$ at price $p$ does not depend on which state of the world has been realized at date 3 . Therefore, if the buyer expects different contract offers in different states of the world, he has to expect these offers to result in identical profits for the seller, i.e., $\left(\hat{v}\left(\theta^{\prime}, \varnothing\right), \hat{p}\left(\theta^{\prime}, \varnothing\right)\right) \neq\left(\hat{v}\left(\theta^{\prime \prime}, \varnothing\right), \hat{p}\left(\theta^{\prime \prime}, \varnothing\right)\right)$ implies $\hat{p}\left(\theta^{\prime}, \varnothing\right)-\hat{c}\left(\theta^{\prime}, \varnothing\right)=\hat{p}\left(\theta^{\prime \prime}, \varnothing\right)-\hat{c}\left(\theta^{\prime \prime}, \varnothing\right)$. Otherwise the seller would have an incentive to deviate from the buyer's expectations by making the most profitable offer in each state of the world. As argued in the text, zero value is always associated with a zero expected price. It follows that in equilibrium the buyer cannot expect spot contracting to result in zero value in any state of the world: with expected profits from spot contracting equal to zero in this case, the seller would have left the market at date 2 to pursue her outside option.

Thus, suppose the buyer expects to obtain value $v_{L}$ for states in some strict subset $\Theta_{L} \subset \Theta$ of the state space, and to obtain value $v_{H}$ for the states in the complementary set $\Theta_{H}=\Theta \backslash \Theta_{L}$. Let $\hat{Q}_{k}=\left|\Theta_{k}\right| / n$ denote the probability with which the buyer expects to obtain value $v_{k}$ with $k \in\{L, H\}$. By the reasoning above, the buyers price expectations have to satisfy $\hat{p}\left(\theta^{\prime}, \varnothing\right)=p_{L}$ for all $\theta^{\prime} \in \Theta_{L}, \hat{p}\left(\theta^{\prime \prime}, \varnothing\right)=p_{H}$ for all $\theta^{\prime \prime} \in \Theta_{H}$, and $p_{L}-c_{L}=p_{H}-c_{H}$. For the buyer's expectations to be consistent with equilibrium, he must expect his utility from both spot contract offers, $\left(v_{L}, p_{L}\right)$ and $\left(v_{H}, p_{H}\right)$, to be at least as high as his utility from spot contracting not taking place, i.e.,

$$
U\left(v_{L}, p_{L} \mid \Lambda(\varnothing)\right)=v_{L}-p_{L}-\lambda \hat{Q}_{H}\left(v_{H}-v_{L}\right) \geq-\lambda\left(\hat{Q}_{L} v_{L}+\hat{Q}_{H} v_{H}\right)
$$

and

$$
\begin{aligned}
U\left(v_{H}, p_{H} \mid \Lambda(\varnothing)\right)=v_{H}- & p_{H}-\lambda \hat{Q}_{L}\left(p_{H}-p_{L}\right) \\
& =v_{H}-p_{L}-\left(1+\lambda \hat{Q}_{L}\right)\left(c_{H}-c_{L}\right) \geq-\lambda\left(\hat{Q}_{L} v_{L}+\hat{Q}_{H} v_{H}\right) .
\end{aligned}
$$

Finally, for the seller not to have an incentive to deviate by slightly increasing the price in states $\theta \in \Theta_{L}$ or states $\theta \in \Theta_{H}$, both (B.1) and (B.2) have to be binding. Since $U\left(v_{L}, p_{L} \mid \Lambda(\varnothing)\right)=$ $U\left(v_{H}, p_{H} \mid \Lambda(\varnothing)\right)$ if and only if

$$
\left(c_{H}-c_{L}\right)-\left(v_{H}-v_{L}\right)=\lambda\left[\hat{Q}_{H}\left(v_{H}-v_{L}\right)-\hat{Q}_{L}\left(c_{H}-c_{L}\right)\right],
$$

this last requirement, however, generically fails to hold because (B.3) is satisfied for at most one value of $\lambda$.

Proof of Lemma 1. Given contract $C_{E, \mathcal{A}}(\bar{p})$, each state $\theta \in \Theta$ is associated with a unique default outcome $\left(v^{D}(\theta, C), c^{D}(\theta, C)\right)$. With our focus on pure strategies, the buyer expects renegotiations in state $\theta$ to result in a particular outcome $(\hat{v}(\theta, C), \hat{c}(\theta, C))$. 
Letting $\Lambda(C)$ denote the buyer' expectations consistent with equilibrium for a given contract $C=C_{E, \mathcal{A}}(\bar{p})$, a first observation is that if renegotiations occur in state $\theta \in \Theta$, then the seller's renegotiation offer makes the buyer just indifferent between the default outcome and the renegotiated outcome, i.e., $U(\hat{v}(\theta, C), \hat{p}(\theta, C) \mid \Lambda(C))=U\left(v^{D}(\theta, C), \bar{p} \mid \Lambda(C)\right)$. If the buyer was not indifferent, then either he would reject the seller's renegotiation offer or the seller could profitably deviate by offering the outcome $(\hat{v}(\theta, C), \hat{c}(\theta, C))$ at a slightly higher price which the buyer nevertheless would accept.

The above observation has two immediate implications. First, if the buyer expects renegotiation to lead to the same value to be implemented in two states with the same default outcome, then he has to expect the same price to be offered by the the seller in renegotiations. Formally, if $\hat{v}\left(\theta^{\prime}, C\right)=\hat{v}\left(\theta^{\prime \prime}, C\right)$ for $\theta^{\prime}, \theta^{\prime \prime} \in \Theta$ with $v^{D}\left(\theta^{\prime}, C\right)=v^{D}\left(\theta^{\prime \prime}, C\right)$, then $\hat{p}\left(\theta^{\prime}, C\right)=\hat{p}\left(\theta^{\prime \prime}, C\right)$. Second, if the buyer expects renegotiations in two states with different default outcomes to result in the same outcome with either strictly higher or strictly lower value than the two default outcomes, then the buyer has to expect renegotiations involving higher differences in value to lead to a larger change in the trade price. Formally, if $\hat{v}\left(\theta^{\prime}, C\right)=\hat{v}\left(\theta^{\prime \prime}, C\right)=0$ for $\theta^{\prime}, \theta^{\prime \prime} \in \Theta$ with $v^{D}\left(\theta^{\prime}, C\right)=v_{L}$ and $v^{D}\left(\theta^{\prime \prime}, C\right)=v_{H}$ or $\hat{v}\left(\theta^{\prime}, C\right)=\hat{v}\left(\theta^{\prime \prime}, C\right)=v_{H}$ for $\theta^{\prime}, \theta^{\prime \prime} \in \Theta$ with $v^{D}\left(\theta^{\prime}, C\right)=0$ and $v^{D}\left(\theta^{\prime \prime}, C\right)=v_{L}$, then $\hat{p}\left(\theta^{\prime}, C\right)>\hat{p}\left(\theta^{\prime \prime}, C\right)$.

Armed with these observations, we can establish that the buyer's expectation in equilibrium about the outcome of renegotiations after state $\theta \in \Theta$ has been realized is fully determined by the default outcome $\left(v^{D}(\theta, C), c^{D}(\theta, C)\right)$. For $k, j \in\{0, L, H\}$, define

$$
\Theta_{\{k \rightarrow j\}}:=\left\{\theta \in \Theta \mid v^{D}(\theta, C)=v_{k}, \hat{v}(\theta, C)=v_{j}\right\}
$$

to be the set of states of the world that share the same default outcome under contract $C$ and that the buyer expects to be identically renegotiated. Due to our focus on pure strategies, $\Theta_{\left\{k \rightarrow j^{\prime}\right\}} \cap$ $\Theta_{\left\{k \rightarrow j^{\prime \prime}\right\}}=\varnothing$ for $j^{\prime} \neq j^{\prime \prime}$. Furthermore, define

$$
Q_{\{k \rightarrow j\}}:=\frac{\left|\Theta_{\{k \rightarrow j\}}\right|}{n} .
$$

From the discussion above, for all $\theta \in \Theta_{\{k \rightarrow j\}}$ the buyer expects the seller to offer the same price $\hat{p}_{\{k \rightarrow j\}}$ in renegotiations, where $\hat{p}_{\{k \rightarrow k\}}=\bar{p}$.

Whenever $\Theta_{\left\{k \rightarrow j^{\prime}\right\}} \neq \varnothing$ and $\Theta_{\left\{k \rightarrow j^{\prime \prime}\right\}} \neq \varnothing$, the buyer expects the seller to be indifferent between offering $v_{j^{\prime}}$ at price $\hat{p}_{\left\{k \rightarrow j^{\prime}\right\}}$ and offering $v_{j^{\prime \prime}}$ at price $\hat{p}_{\left\{k \rightarrow j^{\prime \prime}\right\}}$ if her default is to sell $v_{k}$ at price $\bar{p}$. If this was not the case, i.e. if (w.l.o.g.) $\hat{p}_{\left\{k \rightarrow j^{\prime}\right\}}-c_{j^{\prime}}>\hat{p}_{\left\{k \rightarrow j^{\prime \prime}\right\}}-c_{k^{\prime \prime}}$, then for $\theta \in \Theta_{\left\{k \rightarrow j^{\prime \prime}\right\}}$ the seller could offer $v_{j^{\prime}}$ at a price $\hat{p}_{\left\{k \rightarrow j^{\prime}\right\}}-\varepsilon$ with $\varepsilon>0$ sufficiently small, thereby making the buyer strictly prefer to obtain $v_{j^{\prime}}$ at price $\hat{p}_{\left\{k \rightarrow j^{\prime}\right\}}-\varepsilon$ while at the same time strictly increasing her profits. This indifference of the seller has the following implications for 
the buyer's expectations regarding prices:

$$
\begin{aligned}
& \Theta_{\{H \rightarrow H\}} \neq \varnothing, \Theta_{\{H \rightarrow L\}} \neq \varnothing \Rightarrow \bar{p}-c_{H}=\hat{p}_{\{H \rightarrow L\}}-c_{L} \\
& \Theta_{\{H \rightarrow H\}} \neq \varnothing, \Theta_{\{H \rightarrow 0\}} \neq \varnothing \Rightarrow \bar{p}-c_{H}=\hat{p}_{\{H \rightarrow 0\}} \\
& \Theta_{\{H \rightarrow L\}} \neq \varnothing, \Theta_{\{H \rightarrow 0\}} \neq \varnothing \Rightarrow \hat{p}_{\{H \rightarrow L\}}-c_{L}=\hat{p}_{\{H \rightarrow 0\}} \\
& \Theta_{\{L \rightarrow H\}} \neq \varnothing, \Theta_{\{L \rightarrow L\}} \neq \varnothing \Rightarrow \hat{p}_{\{L \rightarrow H\}}-c_{H}=\bar{p}-c_{L} \\
& \Theta_{\{L \rightarrow H\}} \neq \varnothing, \Theta_{\{L \rightarrow 0\}} \neq \varnothing \quad \Rightarrow \quad \hat{p}_{\{L \rightarrow H\}}-c_{H}=\hat{p}_{\{L \rightarrow 0\}} \\
& \Theta_{\{L \rightarrow L\}} \neq \varnothing, \Theta_{\{L \rightarrow 0\}} \neq \varnothing \Rightarrow \bar{p}-c_{L}=\hat{p}_{\{L \rightarrow 0\}} \\
& \Theta_{\{0 \rightarrow H\}} \neq \varnothing, \Theta_{\{0 \rightarrow L\}} \neq \varnothing \quad \Rightarrow \quad \hat{p}_{\{0 \rightarrow H\}}-c_{H}=\hat{p}_{\{0 \rightarrow L\}}-c_{L} \\
& \Theta_{\{0 \rightarrow H\}} \neq \varnothing, \Theta_{\{0 \rightarrow 0\}} \neq \varnothing \quad \Rightarrow \quad \hat{p}_{\{0 \rightarrow H\}}-c_{H}=\bar{p} \\
& \Theta_{\{0 \rightarrow L\}} \neq \varnothing, \Theta_{\{0 \rightarrow 0\}} \neq \varnothing \quad \Rightarrow \quad \hat{p}_{\{0 \rightarrow L\}}-c_{L}=\bar{p}
\end{aligned}
$$

At the same time, in equilibrium we have $U\left(v_{j^{\prime}}, \hat{p}_{\left\{k \rightarrow j^{\prime}\right\}} \mid \Lambda(C)\right)=U\left(v_{k^{\prime \prime}}, \hat{p}_{\left\{k \rightarrow j^{\prime \prime}\right\}} \mid \Lambda(C)\right)$, i.e., the buyer expects to be indifferent between obtaining $v_{j^{\prime}}$ at price $\hat{p}_{\left\{k \rightarrow j^{\prime}\right\}}$ and obtaining $v_{j^{\prime \prime}}$ at price $\hat{p}_{\left\{k \rightarrow j^{\prime}\right\}}$ if the default is to obtain $v_{k}$ at price $\bar{p}$. It can be shown that this latter indifference of the buyer is characterized by the zeros of a polynomial in $\lambda$ of finite order, and thus, with $\lambda$ being drawn from the interval $(0,1]$, generically does not hold. With the procedure being the same for all relevant cases, we demonstrate the result only for two example cases.

CASE 1: Suppose that $\Theta_{\{H \rightarrow H\}} \neq \varnothing, \Theta_{\{H \rightarrow L\}} \neq \varnothing, \Theta_{\{L \rightarrow L\}} \neq \varnothing, \Theta_{\{0 \rightarrow 0\}} \neq \varnothing$, and $\Theta_{\{H \rightarrow 0\}}=\Theta_{\{L \rightarrow H\}}=\Theta_{\{L \rightarrow 0\}}=\Theta_{\{0 \rightarrow H\}}=\Theta_{\{0 \rightarrow L\}}=\varnothing$. Regarding the relevant price expectations we have $\hat{p}_{\{H \rightarrow H\}}=\hat{p}_{\{L \rightarrow L\}}=\hat{p}_{\{0 \rightarrow 0\}}=\bar{p}$ and $\hat{p}_{\{H \rightarrow L\}}=\bar{p}-\left(c_{H}-c_{L}\right)$. Moreover, we have that $U\left(v_{L}, \hat{p}_{\{H \rightarrow L\}} \mid \Lambda(C)\right)=v_{L}-\left[\bar{p}-\left(c_{H}-c_{L}\right)\right]-\lambda Q_{\{H \rightarrow H\}}\left(v_{H}-v_{L}\right)$ equals $U\left(v_{H}, \bar{p} \mid \Lambda(C)\right)=v_{H}-\bar{p}-\lambda Q_{\{H \rightarrow L\}}\left[\bar{p}-\left(\bar{p}-\left(c_{H}-c_{L}\right)\right)\right]$, or equivalently,

$$
\left(c_{H}-c_{L}\right)-\left(v_{H}-v_{L}\right)=\lambda\left\{Q_{\{H \rightarrow H\}}\left(v_{H}-v_{L}\right)-Q_{\{H \rightarrow L\}}\left(c_{H}-c_{L}\right)\right\} .
$$

If at all, (B.6) holds for at most one value of $\lambda$ and thus generically is not satisfied.

CASE 2: Suppose that $\Theta_{\{H \rightarrow L\}} \neq \varnothing, \Theta_{\{H \rightarrow 0\}} \neq \varnothing, \Theta_{\{L \rightarrow L\}} \neq \varnothing, \Theta_{\{L \rightarrow 0\}} \neq \varnothing, \Theta_{\{H \rightarrow H\}}=$ $\varnothing$, and $\Theta_{\{L \rightarrow H\}}, \Theta_{\{0 \rightarrow H\}}, \Theta_{\{0 \rightarrow L\}}, \Theta_{\{0 \rightarrow 0\}}$ arbitrarily specified. Regarding the relevant price expectations we have $\hat{p}_{\{H \rightarrow 0\}}=\hat{p}_{\{H \rightarrow L\}}-c_{L}<\hat{p}_{\{H \rightarrow L\}}<\bar{p}, \hat{p}_{\{L \rightarrow 0\}}=\bar{p}-c_{L}<\bar{p}=$ $\hat{p}_{\{L \rightarrow L\}}$, and $\hat{p}_{\{L \rightarrow H\}}, \hat{p}_{\{0 \rightarrow H\}}, \hat{p}_{\{0 \rightarrow L\}}, \hat{p}_{\{0 \rightarrow 0\}} \geq \bar{p}$. Remember, from the discussion above, we know that $\hat{p}_{\{H \rightarrow 0\}}<\hat{p}_{\{L \rightarrow 0\}}$. Suppose that $\hat{p}_{\{H \rightarrow L\}}>\hat{p}_{\{L \rightarrow 0\}}$. In equilibrium, we have that $U\left(v_{L}, \hat{p}_{\{H \rightarrow L\}} \mid \Lambda(C)\right)=v_{L}-\hat{p}_{\{H \rightarrow L\}}-\lambda\left(Q_{\{L \rightarrow H\}}+Q_{\{0 \rightarrow H\}}\right)\left(v_{H}-v_{L}\right)-\lambda Q_{\{H \rightarrow 0\}}\left(\hat{p}_{\{H \rightarrow L\}}-\right.$ $\left.\hat{p}_{\{H \rightarrow 0\}}\right)-\lambda Q_{\{L \rightarrow 0\}}\left(\hat{p}_{\{H \rightarrow L\}}-\hat{p}_{\{L \rightarrow 0\}}\right)$ equals $U\left(v_{H}, \bar{p} \mid \Lambda(C)\right)=v_{H}-\bar{p}-\lambda Q_{\{H \rightarrow 0\}}(\bar{p}-$ $\left.\hat{p}_{\{H \rightarrow L\}}\right)-\lambda Q_{\{L \rightarrow 0\}}\left(\bar{p}-\hat{p}_{\{L \rightarrow 0\}}\right)-\lambda Q_{\{H \rightarrow L\}}\left(\bar{p}-\hat{p}_{\{H \rightarrow L\}}\right)$ such that

$$
\hat{p}_{\{H \rightarrow L\}}=\bar{p}-\frac{1+\lambda\left(Q_{\{L \rightarrow H\}}+Q_{\{0 \rightarrow H\}}\right)}{1+\lambda\left(Q_{\{H \rightarrow 0\}}+Q_{\{L \rightarrow 0\}}+Q_{\{H \rightarrow L\}}\right)}\left(v_{H}-v_{L}\right),
$$

and in consequence,

$$
\hat{p}_{\{H \rightarrow 0\}}=\hat{p}_{\{H \rightarrow L\}}-c_{L}=\bar{p}-c_{L}-\frac{1+\lambda\left(Q_{\{L \rightarrow H\}}+Q_{\{0 \rightarrow H\}}\right)}{1+\lambda\left(Q_{\{H \rightarrow 0\}}+Q_{\{L \rightarrow 0\}}+Q_{\{H \rightarrow L\}}\right)}\left(v_{H}-v_{L}\right),
$$


Given these beliefs are feasible in the sense that $\hat{p}_{\{H \rightarrow 0\}}<\hat{p}_{\{L \rightarrow 0\}}<\hat{p}_{\{H \rightarrow L\}}$, then $U\left(0, \hat{p}_{\{H \rightarrow 0\}} \mid \Lambda(C)\right)=-\hat{p}_{\{H \rightarrow 0\}}-\lambda\left(Q_{\{H \rightarrow L\}}+Q_{\{L \rightarrow L\}}+Q_{\{0 \rightarrow L\}}\right) v_{L}-\lambda\left(Q_{\{L \rightarrow H\}}+Q_{\{0 \rightarrow H\}}\right) v_{H}$ equals $U\left(v_{H}, \bar{p} \mid \Lambda(C)\right)=v_{H}-\bar{p}-\lambda Q_{\{H \rightarrow 0\}}\left(\bar{p}-\hat{p}_{\{H \rightarrow 0\}}\right)-\lambda Q_{\{H \rightarrow L\}}\left(\bar{p}-\hat{p}_{\{H \rightarrow L\}}\right)-\lambda Q_{\{L \rightarrow 0\}}(\bar{p}-$ $\left.\hat{p}_{\{L \rightarrow 0\}}\right)$, which is equivalent to

$$
\begin{array}{r}
\left(v_{H}-c_{L}\right)+\lambda\left(Q_{\{H \rightarrow 0\}}+Q_{\{L \rightarrow 0\}}+Q_{\{H \rightarrow L\}}\right)\left(v_{H}-c_{L}\right)= \\
{\left[1+\lambda\left(Q_{\{L \rightarrow H\}}+Q_{\{0 \rightarrow H\}}\right)\right]\left[1+\lambda\left(Q_{\{H \rightarrow 0\}}+Q_{\{H \rightarrow L\}}\right)\right]\left(v_{H}-v_{L}\right)} \\
-\lambda\left[1+\lambda\left(Q_{\{H \rightarrow 0\}}+Q_{\{L \rightarrow 0\}}+Q_{\{H \rightarrow L\}}\right)\right]\left\{\left(Q_{\{H \rightarrow L\}}+Q_{\{L \rightarrow L\}}+Q_{\{0 \rightarrow L\}}\right) v_{L}\right. \\
\left.+\left(Q_{\{L \rightarrow H\}}+Q_{\{0 \rightarrow H\}}\right) v_{H}-\left(Q_{\{H \rightarrow 0\}}+Q_{\{L \rightarrow 0\}}\right) c_{L}\right\}
\end{array}
$$

If at all, (B.9) holds for at most two values of $\lambda$ and thus generically is not satisfied.

\section{Definition of the Equilibrium Concepts}

Definition 1. A Subgame Pefect Personal Equilibrium (SP-PE) is a strategy profile for each player $P \in\left(S_{1}, S_{2}, B\right)$ with the property that $(i)$ in no subgame can any player $P$ do better by choosing a different strategy, keeping the strategies of all other players fixed, (ii) player B's strategy constitutes a personal equilibrium-i.e., correctly anticipating equilibrium play at date 1 , which determines B's reference point in the value and the money dimension, it is optimal for the buyer to behave according to this strategy at date 5. More precisely, at each date players behave as follows:

5.) In the subgame following the acceptance of $S_{i}$ 's long-term contract offer, the buyer decides whether or not to accept the renegotiation offer proposed by $S_{i}$. In the subgame following the buyer's rejection of all long-term contract offers, the buyer decides whether or not to accept exactly one of the proposed spot contracts. The buyer's decision maximizes his utility induced by the reference point formed at date 1 . When being indifferent between accepting and rejecting an offer, the buyer accepts the offer. When being indifferent which offer to accept, the buyer chooses the offer that gives him the highest value in the value dimension. Further ties are broken by the flip of a fair coin by B.

4.) If seller $S_{i}$ 's long-term contract was accepted at date 1 , she makes a renegotiation offer $\left(x^{R}, p^{R}\right) \in \mathcal{X} \times \mathbb{R}$. The seller makes a profit-maximizing offer, taking $B$ 's behavior at date 5 into account-in particular, the seller correctly anticipates the personal equilibrium played by the buyer.

If no long-term contract was concluded at date 1, a seller active in the market proposes a spot contract $\left(x^{\text {spot }}, p^{\text {spot }}\right) \in \mathcal{X} \times \mathbb{R}$. Active sellers make their offers simultaneously and non-cooperatively. Each seller makes an offer that maximizes her expected profitscorrectly anticipating the other seller's offer and the buyer's behavior at date 5-again, correctly anticipating the personal equilibrium played by the buyer. 
3.) Nature draws the state of the world $\theta_{i} \in \Theta$, where each state $\theta_{i}$ with $i=1, \ldots, n$ is drawn with probability $1 / n$.

2.) Only in the subgame following the rejection of all long-term contract offers at date 1, there is a decision to be made at date 2. Both sellers simultaneously and non-cooperatively decide whether or not to stay in the market. Each seller takes the other seller's strategy as given and correctly anticipates subsequent play. A seller leaving the market realizes a profit of $\bar{\pi}$.

1.) The buyer observes the contracts offered by the two sellers. He either accepts exactly one contract or he rejects both contracts. In evaluating each option the buyer correctly takes into account subsequent play by the sellers. Moreover, the buyer's rational expectations about subsequent play for each option form a reference point if he executes this option. This reference point shapes the buyer's utility at date 5 and therefore influences his behavior at date 5. The buyer's reference plan has to be consistent with his behavior at date 5-i.e., the plan has to constitute a personal equilibrium (PE). For each available option the buyer forms a credible reference plan. For option $C \in\left\{C_{E, \mathcal{A}}(\bar{p}), \varnothing\right\}$, we call any such credible plan a subgame perfect personal equilibrium (SP-PE) for the subgame beginning with the choice of option $C$. The buyer then selects at date 1 the option that maximizes his expected utility for these given credible reference plans. If the buyer accepts the contract offered by $S_{i}$, then $S_{j}, j \neq i$, leaves the market and realizes a profit of $\bar{\pi}$. If both offers are rejected, the two sellers stay in the market.

0.) Each seller proposes simultaneously and non-cooperatively a long-term contract $C_{E, \mathcal{A}}(\bar{p})$ to the buyer, thereby taking the other seller's offer as given. In equilibrium, each seller's expectation regarding the other seller's offer is correct. In addition, each seller correctly anticipates subsequent play of all players. In particular, each seller correctly anticipates - for any feasible contract offer - the personal equilibrium played by the buyergiven that the buyer accepts this contract offer or that he rejects all contract offers.

Definition 2. A Subgame Pefect Preferred Personal Equilibrium (SP-PPE) is a Subgame Perfect Personal Equilibrium, where the buyer chooses at date 1 for each available option the credible plan that maximizes his expected utility-i.e, the preferred personal equilibrium. Date 1 is modified as follows:

1.) The buyer observes the contracts offered by the two sellers. He either accepts exactly one contract or he rejects both contracts. In evaluating each option the buyer correctly takes into account subsequent play by the sellers. Moreover, the buyer's rational expectations about subsequent play for each option form a reference point if he executes this option. This reference point shapes the buyer's utility at date 5 and therefore influences his date 5 behavior. The buyer's reference plan has to be consistent with his date 5 behaviori.e., the plan has to constitute a personal equilibrium (PE). For each available option the buyer selects the plan that maximizes his expected utility among all credible plans-i.e., 
the preferred PE plan (PPE). For option $C \in\left\{C_{E, \mathcal{A}}(\bar{p}), \varnothing\right\}$, we call the most preferred credible plan the subgame perfect preferred personal equilibrium (SP-PPE) for the subgame beginning with the choice of option $C$. The buyer then selects at date 1 the option that maximizes his expected utility for these preferred personal equilibrium plans. If the buyer accepts the contract offered by $S_{i}$, then $S_{j}, j \neq i$, leaves the market and realizes a profit of $\bar{\pi}$. If both offers are rejected, the two sellers stay in the market. 